\title{
Post traumatic stress disorder and substance use disorder
}

Citation for published version (APA):

Gielen, N. J. N. (2016). Post traumatic stress disorder and substance use disorder: symptom interplay and treatment implications. [Doctoral Thesis, Maastricht University]. Maastricht University. https://doi.org/10.26481/dis.20160429ng

Document status and date:

Published: 01/01/2016

DOI:

$10.26481 /$ dis.20160429ng

Document Version:

Publisher's PDF, also known as Version of record

\section{Please check the document version of this publication:}

- A submitted manuscript is the version of the article upon submission and before peer-review. There can be important differences between the submitted version and the official published version of record.

People interested in the research are advised to contact the author for the final version of the publication, or visit the DOI to the publisher's website.

- The final author version and the galley proof are versions of the publication after peer review.

- The final published version features the final layout of the paper including the volume, issue and page numbers.

Link to publication

\footnotetext{
General rights rights.

- You may freely distribute the URL identifying the publication in the public portal. please follow below link for the End User Agreement:

www.umlib.nl/taverne-license

Take down policy

If you believe that this document breaches copyright please contact us at:

repository@maastrichtuniversity.nl

providing details and we will investigate your claim.
}

Copyright and moral rights for the publications made accessible in the public portal are retained by the authors and/or other copyright owners and it is a condition of accessing publications that users recognise and abide by the legal requirements associated with these

- Users may download and print one copy of any publication from the public portal for the purpose of private study or research.

- You may not further distribute the material or use it for any profit-making activity or commercial gain

If the publication is distributed under the terms of Article $25 \mathrm{fa}$ of the Dutch Copyright Act, indicated by the "Taverne" license above, 


\title{
Posttraumatic Stress Disorder and Substance Use Disorder \\ Symptom Interplay and Treatment Implications
}

\author{
Nele Gielen
}




\section{POSTTRAUMATIC STRESS DISORDER AND SUBSTANCE USE DISORDER:}

SYMPTOM INTERPLAY AND TREATMENT IMPLICATIONS

Nele Gielen 
Cover: painting by Karin Veldkamp

Printed by

(C) 2015 Nele Gielen Maastricht

ISBN:

All rights are reserved. No parts of this book may be reproduced or transmitted in any form by any means, without written permission from the holder of the copyright. 


\section{POSTTRAUMATIC STRESS DISORDER AND SUBSTANCE USE DISORDER:}

\section{SYMPTOM INTERPLAY AND TREATMENT IMPLICATIONS}

PROEFSCHRIFT

ter verkrijging van de graad van doctor aan de Universiteit Maastricht, op gezag van de Rector Magnificus, Prof. dr. L.L.G. Soete volgens het besluit van het College van Decanen, in het openbaar te verdedigen op

door

Nele Joyce Nettie Gielen 


\section{Promotor}

Prof. dr. Anita Jansen

\section{Copromotoren}

Dr. Remco C. Havermans

Dr. Chantal Nederkoorn

\section{Beoordelingscommissie}

Prof. dr. Corine de Ruiter (voorzitter; Maastricht University)

Prof. dr. David Bernstein (Maastricht University)

Prof. dr. Dike van de Mheen (Maastricht University)

Prof. dr. Geert Dom (Universiteit Antwerpen)

Dr. Marisol Voncken (Maastricht University)

The research presented in this dissertation has been made possible with the support of the mental health institute Mondriaan and the faculty of Psychology and Neuroscience of Maastricht University, both located in the Netherlands. 


\section{CONTENTS}

CHAPTER 1 General Introduction - p. 7

CHAPTER 2 Prevalence of PTSD among Patients with Substance Use Disorder - p. 19

CHAPTER $3 \quad$ Craving after Trauma and Stress Cue Exposure - p. 35

CHAPTER 4 Perceptions of Clinicians about the Comorbidity between PTSD and Substance Use Disorder - p. 52

CHAPTER 5 Coping skills, Motives for Substance Use and Symptom Interplay: how Patients with Substance Use Disorder and PTSD experience their Reality - p. 71

CHAPTER 6 Patients speaking: how they perceive the Relationship between Trauma, Substance Abuse, Craving, and Relapse - p. 86

CHAPTER 7 General Discussion - p. 98

Summary-p. 118

References - p. 122 



\section{Chapter 1}

General Introduction 
Kevan Lyons - I am blind to beauty

"I am blind to beauty for I have seen the ugliness of war, my heart discard my soul's an open sore, my spirits broken and my body is not well, for I have seen the smoke and fire and passed through the gates of hell,

I've held a dying man grasping for last breath

and been surrounded by the taste of death and the smell of fear,

I've buried both friend and foe in fields where no crops will ever grow, there is no honor in taking of a life, and I have done so with my rifle and my knife, and I do not sleep well at night, for in my dreams I still fight, and the enemy I see is a soldier... and its me." 
Homer, ancient Greek author of the Iliad and the Odyssey, describes in his fourth book of the Odyssey how the young Telemachus is searching for his father Odysseus. In the course of this quest Telemachus is invited by Menelaus, king of Sparta, and his wife Helena. During this encounter the invitees experience intense negative emotions, triggered by the remembrance of the atrocities of the Trojan War. Helena decides to add a 'ne-penthes' (literally a 'banisher of sorrow') to the wine. Homer describes this remedy as a substance that is able to quell all sorrows with forgetfulness, even if one had witnessed the killing of his own dear brother or son. Later commentators speculated upon the nature of this substance and agreed that it is conceivable that Helena mixed opium in the wine of her guests (Odyssey, book 4, v. 219-221). This old work of poetry indicates that the use of substances to deal with emotional pain caused by trauma is neither new nor irrational. Despite this, it was not until 1980 that Lacoursiere, Godfrey, and Ruby published a first article concerning the apparent comorbidity between posttraumatic stress disorder (what was then called traumatic neurosis) and alcoholism.

The central theme of the present dissertation concerns studies examining the role of trauma and posttraumatic stress disorder (PTSD) in its co-existence with and the treatment of substance use disorder (SUD). This chapter provides an overview of the characteristics of patients with SUD/PTSD comorbidity, the existing theories about the comorbidity, the treatment, the guidelines and daily clinical practice. Further, the research questions of the present dissertation are introduced and an outline of the dissertation is provided.

\section{Characteristics of the SUD/PTSD comorbidity}

According to DSM -5 "a SUD describes a problematic pattern of using alcohol or another substance that results in impairment in daily life or noticeable distress". PTSD, on the other hand, is characterized by the persistent re-experiencing, the avoidance of trauma triggers, negative alterations in cognitions and mood, and hyperarousal in response to a direct or indirect traumatic event (5th ed.; DSM-5 APA, 2013).

SUD and PTSD are two severe and often long-lasting psychiatric disorders that frequently cooccur. Since Lacoursiere et al. (1980), various studies were published describing the heightened prevalence rate of PTSD and trauma exposure among SUD patients. The prevalence of current PTSD in SUD patients is around three times higher than in the general population, and nearly all SUD patients ( 9 out of 10 ) have been exposed to some form of trauma (Bonin, Norton, Asmundson, Dicurzio, \& Pidlubney, 2000; Dansky, Saladin, Coffey, \& Brady, 1997; Driessen et al., 2008; Farley, Golding, Young, Mulligan, \& Minkoff, 2004; Kessler et al., 2005; Ouimette, Coolhart, Funderburk, Wade, \& Brown, 2007; Read, Brown, \& Kahler, 2004; Reynolds et al., 2005). SUD is not the only disorder found to be comorbid 
with PTSD, major depressive disorder, anxiety disorders, and borderline personality disorder also share a high comorbidity with PTSD (Pagura, Stein, Bolton, Cox, \& Sareen, 2010; Spinhoven, Penninx, van Hemert, de Rooij, \& Elzinga, 2014).

Previous studies emphasize the vulnerability of patients with the SUD/PTSD comorbidity (Back, Sonne, Killeen, Dansky, \& Brady, 2003; Dube, Anda, Feliti, Chapman, Williamson, \& Giles, 2001; Evren, C., Evren, B., Dalbudak, Ozcelik, \& Oncy, 2009; Mills, Linskey, Teesson, Ross, \& darke, 2005; Mills, Teesson, Ross, Darke, \& Shanahan, 2005; Ouimette, Brown, \& Najavits, 1998; Pirard, Sharon, Kang, Angarita, \& Gastfriend, 2005; Read et al., 2004; Simpson \& Miller, 2002). Compared to those who have SUD only, patients with SUD and comorbid PTSD have a history of more alcohol and drug use, have more prior treatment attempts, have a greater number of mental health treatment sessions, and experience higher levels of craving, distress, and general psychiatric symptoms. When comorbid PTSD is left untreated, SUD patients have a higher risk for relapse of addictive behavior, as compared to patients with SUD only. These abovementioned characteristics are indicative for the vulnerability of this patient group. Again, note that this is a sizeable group of vulnerable patients. More than a quarter of all SUD patients in treatment are thought to have comorbid PTSD. For example, in The Netherlands, there are approximately 30,000 patients in treatment for alcohol dependence (IVZ, 2014), meaning that 7,500 of these alcohol dependent patients have comorbid PTSD. To improve treatment outcome of patients with comorbid SUD and PTSD it is thus important to more closely examine the role of PTSD in these patients.

\section{Theories explaining the SUD/PTSD comorbidity}

Different models for explaining the comorbidity between SUD and PTSD have been put forward. The models can be divided into three clusters: (1) theories assuming that PTSD precedes SUD, (2) theories assuming that SUD precedes PTSD, and (3) theories assuming that a third factor explains the comorbidity, including a maintenance model based on learning theory.

PTSD precedes SUD. It has often been proposed that PTSD is a risk factor for developing further psychopathology, including substance dependence. This sequence of PTSD preceding SUD has indeed been found in both retrospective and prospective studies (Berenz \& Coffey, 2012). A well-known model in this line of thinking is the self-medication hypothesis (Khantzian, 1997; Leeies, Pagura, Sareen, \& Bolton, 2010; Ouimette, Read, Wade, \& Tirone, 2010). This hypothesis states that individuals use substances to dull PTSD symptoms. A patient suffering from posttraumatic hyperarousal might then use alcohol to feel more at ease in social situations. Substance use thus functions as a maladaptive coping strategy. In 2010, Ouimette and colleagues prospectively examined associations between PTSD and SUD 
symptoms. Their findings support the self-medication hypothesis in that PTSD symptom fluctuations predicted variations in SUD symptoms.

A related model explaining how PTSD precedes SUD is the chemical dissociation hypothesis (Langeland, 2009). According to this model, PTSD patients typically cope with their childhood trauma by trying to dissociate: patients then experience a sense of detachment from themselves, their feelings, or their consciousness. However, some patients do not follow this typical path of psychological dissociation, but induce emotional detachment through the use of chemicals; that is, drugs or alcohol (Klanecky, Harrington, \& McChargue; 2008; Langeland, Draijer, \& van den Brink, 2002; Somer, Altus, \& Ginzburg, 2010; Van Den Bosch, Verheul, Langeland, \& Van Den Brink, 2003). Note that in this latter sense, the chemical dissociation hypothesis is indistinguishable from the self-medication hypothesis. Both models assume that SUD/PTSD patients use substances to alleviate PTSD symptoms.

The final model in the first cluster is the dysfunctional stress reaction hypothesis (Hien, Cohen, \& Campbell, 2005). According to this model neuroanatomical alterations may occur as a result of early childhood trauma: an inhibition of the medial prefrontal cortex, an increased activation of the amygdala, and a reduction of the volume of the hippocampus. On a physiological level, a hyperreactive hypothalamic-pituitary-adrenal axis is noticed in patients with repeated childhood maltreatment (Lupien, McEwen, Gunnar, \& Heim, 2009). These neuroanatomical and physiological changes can cause problems in self-regulation and impulse-control, which in turn can lead to alcohol or drug abuse (Langeland, 2009).

SUD precedes PTSD. The second cluster of models suggests a pathway in which SUD precedes trauma exposure and PTSD. The high-risk hypothesis assumes that substance abusing individuals lead a more risky lifestyle, exposing themselves to potentially traumatic experiences, like accidents, violence, or witnessing an overdose (McCauley, Killeen, Gros, Brady, \& Back, 2012). There is evidence that substance use, particularly alcohol abuse, is a risk factor for being exposed to trauma, possibly leading to PTSD (see e.g., Gross, Winslett, Roberts, \& Gohm, 2006).

The susceptibility hypothesis also proposes that PTSD follows SUD. According to this model, SUD can lead to PTSD because chronic substance users experience increased arousal and anxiety (e.g. during withdrawal), and have less adaptive emotion regulating skills rendering them vulnerable to developing PTSD after a traumatic exposure (Haller \& Chassin, 2014; McCauley et al., 2012).

Third factor. According to the liability model, shared vulnerability, or third factor hypothesis common factors play a role in the SUD/PTSD comorbidity. Variables that might independently lead to both the development of SUD and PTSD have been studied: pre-trauma family adversity, biological 
vulnerability (e.g. common genetic liability, hyperactivity of dopamine transmission), poor coping skills, trait-level neuroticism, and anxiety sensitivity (Berenz \& Coffey, 2012; Coffey, Read, \& Norberg, 2008; Haller \& Chassin, 2014; Langeland, 2009; van Dam, Vedel, Ehring, \& Emmelkamp, 2012).

\section{Empirical support for the presented theories}

The self-medication hypothesis is the model that dominates the SUD/PTSD research field, probably because it has strong face validity. But the empirical evidence for this hypothesis is also strong (Coffey et al., 2002; Coffey et al., 2010; Nosen et al., 2012; Saladin et al., 2003). Haller and Chassin (2014) utilized longitudinal data to test the validity of four hypotheses (the self-medication hypothesis, the high-risk hypothesis, the susceptibility hypothesis, and the shared vulnerability hypothesis). They found that PTSD symptoms predicted higher levels of addiction-related problems and that PTSD symptoms mediated the effect of pre-trauma family risk factors on SUD, providing the strongest support for the self-medication hypothesis.

\section{A maintenance model: the learning theoretical view}

It should be noted that the models described above are not mutually exclusive. It is quite conceivable that in some patients SUD preceded PTSD and that in other patients PTSD preceded SUD. In both cases, however, PTSD symptoms can be thought to play a significant role in maintaining the SUD (Berenz \& Coffey, 2012; van Dam et al., 2012). Frequent drug or alcohol use then allows for multiple learning trials in which stress associated with traumatic intrusions becomes associated with the rewarding effects of substance use. According to this learning theoretical view, stress functions as a Pavlovian conditioned drug stimulus (or CS) signaling an unconditioned drug reward stimulus (US). Moreover, to the extent that drug intake itself alleviates PTSD symptoms, substance use is negatively reinforced, hence promoting drug intake even in the absence of stress. Cue-reactivity studies have suggested a link between traumatic memories and the need to dampen the induced emotions with the use of substances. What these studies found is that SUD/PTSD patients experience increased craving after the patients were exposed to personalized trauma cues (Coffey, Saladin, Drobes, Brady, Dansky, \& Kilpatrick, 2002; Coffey, Schumacher, Stasiewicz, Henslee, Baillie, \& Landy, 2010; Nosen, Nillni, Berenz, Schumacher, Stasiewicz, \& Coffey, 2012; Saladin, Drobes, Coffey, Dansky, Brady, \& Kilpatrick, 2003).

Without shedding light on the chicken-or-the-egg problem of comorbid SUD/PTSD, these findings nonetheless strengthen the view that PTSD symptoms play a crucial role in the maintenance and relapse 
of SUD. Figure 1 displays a model of how different associative learning processes are viewed to interact motivating substance use in SUD persons suffering from comorbid PTSD.

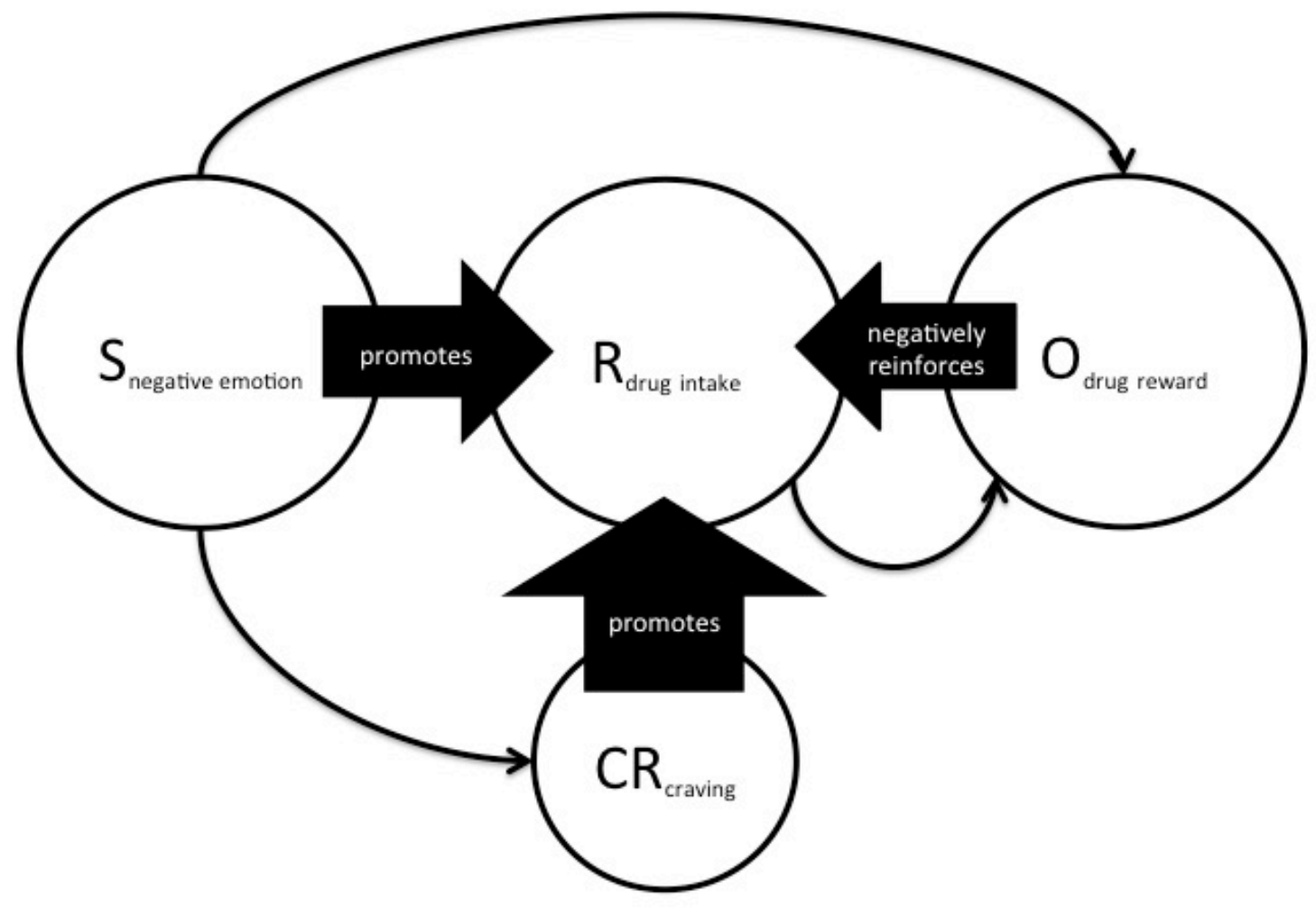

Figure 1. According to the learning theoretical model of comorbid SUD/PTSD suggested in this dissertation, PTSD provides for frequent and strong experiences of negative emotions (e.g., stress, emotional pain, and anxiety) that function as salient internal cues or stimuli (S) that promote coping behavior. Drug (or alcohol) use is a typical form of maladaptive coping that is a purposeful act or response $(R)$ being negatively reinforced by the outcome $(0)$ of drug intake. In parallel, negative emotions become directly associated with $\mathrm{O}$ and as a consequence of this Pavlovian conditioning process become endowed with an incentive value. As such, negative emotions can come to elicit a strong conditioned craving response (CR), which in turn promotes drug (or alcohol) use.

When SUD/PTSD patients continually use substances after experiencing PTSD symptoms, the normal process of fear extinction cannot occur, meaning that substance use can in fact prolong the PTSD symptoms. It is expected that SUD/PTSD patients show increased craving after exposure to traumatic 
cues. Moreover, it is probable that not only exposure to traumatic triggers induce craving, but that stress cues exert this same influence. It is conceivable that when SUD patients with high PTSD severity have learned that substance use leads to a temporarily relief of PTSD symptoms, this powerful connection is widened, such that stress triggers also lead to craving and/ or substance use especially in patients with SUD/PTSD comorbidity. These assumptions are tested in this dissertation.

\section{Treatment}

Over the last decade, a large body of research on the treatment of SUD patients with comorbid PTSD has accumulated. SUD/PTSD treatments are characterized by two distinctive qualities: sequential versus integrated treatment, and past-focused versus present-focused treatment. Sequential treatment was (and perhaps still is) the prevailing standard for some time. Patients were referred to PTSD specialists only after a certain period of sobriety. However, integrated treatment focusing on both disorders simultaneously seems to provide a better outcome than treatment focusing on just one disorder at a time (Berenz \& Coffey, 2012; Henslee \& Coffey, 2010; Hien, Cohen, Miele, Litt, \& Gapstick; 2004; Hildebrand, Behrendt, \& Hoyer, 2014; McCauley et al., 2012; Mills et al., 2012; Najavits, Gallop, \& Weiss, 2006; Najavits \& Hien, 2013).

In present-focused trauma therapy the main focus is on symptom reduction, coping skills training and psycho-education, without targeting the traumatic memories directly. Examples are Seeking Safety (SS), Helping Women Recover plus Beyond Trauma (HWR/BT), Integrated Cognitive Behavior Therapy for PTSD and SUD (ICBT), Addictions and Trauma Recovery Integrated Model (ATRIUM), Trauma Adaptive Recovery Group Education and Therapy or Trauma Affect Regulation: Guidelines for Education and Therapy (TARGET), Transcend, CBT for PTSD in SUD treatment, and Trauma Recovery and Empowerment Model (TREM). In past-focused trauma therapy, exactly the opposite is done; that is, the traumatic experience is then the main focus of the therapy. Past-focused SUD/PTSD therapies are Concurrent Treatment of PTSD and Cocaine Dependence (CTPCD) or Concurrent treatment of PTSD and SUD using prolonged Exposure (COPE), Creating Change (CC), and Substance Dependence PTSD Therapy (SDPT).

Recently, review articles were published on the treatment of co-occurring SUD and PTSD, (Berenz \& Coffey, 2012; McCauley et al., 2012; Najavits \& Hien, 2013; van Dam et al., 2012). Despite the occurrence of several methodological problems of the reviewed studies, some important conclusions were drawn by the reviewers. SUD/PTSD treatment duration is long, taking up to 40 hours of face to face treatment. Patient satisfaction is mostly high, although drop-out rates are not to be 
underestimated, especially in the past-focused treatments. Both present- and past-focused studies show symptom reduction and seem promising. The past-focused therapies show somewhat more symptom reduction. The reviews further underline the need to treat SUD and PTSD simultaneously and in an integrated manner (Berenz \& Coffey, 2012; McCauley et al., 2012; Najavits \& Hien, 2013; van Dam et al., 2012). The reviewed studies further show that when SUD and PTSD are treated simultaneously, PTSD symptoms in particular diminish, while SUD symptoms seem most difficult to reduce. However, despite the accumulating evidence suggesting that an integrated treatment of comorbid SUD/PTSD has benefit relative to sequential treatment, few substance dependent treatment centers have implemented an integrated treatment approach, leaving PTSD in most cases untreated during SUD treatment (GloverGraf \& Janikowski, 2001; Najavits, Sullivan, Schmitz, Weiss, \& Lee, 2004; Young, Rosen, \& Finney, 2005). There is a clear gap between science and clinical practice here. It is not clear exactly why integrated treatment is not frequently offered, or how to facilitate implementing integrated treatment of SUD and co-occurring PTSD.

\section{Guidelines}

In the last years, guidelines, focusing on the SUD/PTSD comorbidity and aimed to improve the quality of healthcare, have been developed. These guidelines are by and large consistent with the drawn conclusions in the review articles. The VA/DoD developed guideline recommendations for co-occurring SUD and PTSD (Kivlahan \& Kaysen, 2012) are developed In the USA, focusing on veterans with combatrelated PTSD:

1. All patients diagnosed with PTSD should receive comprehensive assessment for SUD, including nicotine dependence.

2. Recommend and offer cessation treatment to patients with nicotine dependence. (McFall et al., 2010)

3. Patients with SUD and PTSD should be educated about the relationship between PTSD and substance abuse. The patient's prior treatment experience and preference should be considered since no single intervention approach for the co-morbidity has yet emerged as the treatment of choice.

4. Treat the disorders concurrently. Treat other concurrent SUD consistent with the SUD guidelines including concurrent pharmacotherapy.

- Addiction-focused pharmacotherapy should be discussed, considered, and offered, if indicated. 
- This pharmacotherapy should be monitored.

5. Provide multiple services in the most accessible setting that will promote engagement, and coordination of care, for both SUD and PTSD (integrated care).

6. Reassess responses to treatment for SUD periodically and systematically, using standardized and valid self-report instruments and laboratory tests. Indicators of SUD treatment response include ongoing substance use, craving, side effects of medication, emerging symptoms, etc. (measurement-based care)

7. There is insufficient evidence to recommend for or against any specific psychosocial approach to addressing PTSD that is comorbid with SUD.

In the Netherlands, guidelines were developed on the treatment of comorbid SUD and anxiety disorders. The SUD/PTSD comorbidity is one of the chapters in this extensive manuscript (Snoek, Wits, Meulders, \& van de Mheen, 2012). It is underlined that in case of comorbid SUD and PTSD, clinicians need not to follow the general guidelines for dual diagnosis. Instead, PTSD needs to be a focus in SUD treatment, during and immediately after detoxification. During the integrated treatment the PTSD treatment should be emphasized. The usual treatment advices for SUD treatment can be followed, except that exposure based therapies might be less efficient when the patient is intoxicated. A final advice in this guideline is never to treat comorbid PTSD and SUD with pharmacotherapy alone.

The International Society for Traumatic Stress Studies (ISTSS), which holds international appeal, developed PTSD treatment guidelines in 2005 (Foa, Keane, Friedman, \& Cohen, 2008). One chapter describes shortly the quality of existing SUD/PTSD treatments. The present-focused SS is indicated as a level A treatment (suggesting its superiority), whereas the present-focused treatments Transcend and TREM, and the past-focused treatments CTPCD and CDPT all received C-levels. A specific SUD/PTSD guideline was developed in 2012 by the Substance Abuse and Mental Health Services Association (SAMHSA), another esteemed organization in the USA. This guideline specifically addresses pharmacotherapy in patients with comorbid opioid use disorder and PTSD and advices to assess, screen for and, if necessary, treat both disorders simultaneously when a patient presents himself with only PTSD or only SUD. The 2004 American Psychiatric Association (APA) practice guidelines of PTSD and Acute Stress Disorder acknowledge the complexity of treating comorbid SUD and PTSD and posit that substance use should be addressed as part of the PTSD treatment, regardless of whether the criteria for SUD are met. Likewise, in the 2006 APA guideline for SUD, integrated treatment is advised and empirical evidence for both present-focused and past-focused integrated treatment approaches is given. The 
prescription of benzodiazepines is firmly discouraged. Overall, the guidelines underline the importance of integrated SUD/PTSD treatment.

\section{Aims of the dissertation}

The aim of the present dissertation is threefold. The first aim is to study the prevalence of PTSD in SUD patients and to analyze the characteristics of patients with the SUD/PTSD comorbidity. Although previous studies have shown an increased prevalence of PTSD in SUD patients (Bonin et al., 2000; Dansky et al., 1997; Driessen et al., 2008; Farley et al., 2004; Kessler et al., 2005; Ouimette et al., 2007; Read et al., 2004; Reynolds et al., 2005), no study had directly compared the prevalence of current PTSD and trauma exposure in a SUD population with the prevalence of current PTSD and trauma exposure in the general population using the same diagnostic tool. Since previous studies found substantial differences in PTSD prevalence rates depending on which diagnostic tool was used (Dragan \& LisTurlejska; 2007), it is deemed important to compare prevalence rates with identical questionnaires within the same study. Furthermore, previous studies examining socio-economical and clinical characteristics provided contradicting results with regard to characteristics of SUD/PTSD patients (Bonin et al., 2000; Driessen et al., 2008; Mills, Linskey, et al., 2005; Mills, Teesson, Ross, \& Peters, 2006; Peirce, Kindbom, Waesche, Yuscavage, \& Brooner, 2008; Ouimette, Goodwin, \& Brown, 2006). More research is thus needed to explore the prevalence and the characteristics of SUD/PTSD patients.

A second aim of the present dissertation is to study how the craving response of SUD/PTSD patients interacts with trauma and stress cues. More specifically, the assumption based on the learning theoretical view outlined above is tested that both trauma and stress cues will induce craving in SUD/PTSD patients. If indeed craving is not solely related to trauma triggers, but also to more general stressors, this might have important treatment implications.

The third and final aim of the dissertation is to increase our understanding of why the implementation of integrated SUD/PTSD treatment hitherto has been unsuccessful. When specific factors for this failure can be indicated, suggestions for how to remove these blockades can be provided.

\section{Outline of the dissertation}

The present dissertation will start with a chapter (2) examining the prevalence of current PTSD and trauma exposure in an SUD patient sample, versus the prevalence of PTSD and trauma exposure in the general population. By comparing the rate of PTSD measured with and without using a standardized questionnaire, it was assessed whether PTSD can be detected in SUD treatment centers when relying on 
clinical judgment alone. A last goal of this chapter was to gain more insight in the socio-economic and psychiatric characteristics of patients with SUD/PTSD. This chapter gives a good understanding of the magnitude and vulnerability of the subgroup of patients with SUD/PTSD and gives a first insight in why PTSD treatment is often overlooked or disregarded when treating SUD patients.

In Chapter 3, trauma and stress cue induced craving is studied. The chapter describes an experiment in which SUD patients with differing PTSD severity were exposed to personalized stress and trauma cues while craving levels were assessed. It was tested whether SUD/PTSD patients showed craving as response to trauma cues and in addition, if these cued cravings are specific for trauma cues or also more general to stress cues.

In the following chapter (4) the focus is on the perceptions of clinicians with regard to PTSD in SUD patients. In the described qualitative study we questioned the current procedures, the responsibilities, and the possible obstacles related to the assessment, the diagnosis, and the treatment of SUD/PTSD patients. This chapter shows clear obstacles related to the implementation of integrated treatment.

The fifth chapter studies the views of SUD patients with different levels of PTSD severity. Patients were surveyed about their ideas regarding the link between the start of their addiction and their past trauma, they were questioned about their motives for using substances, and lastly, they filled out questionnaires about the relationship between PTSD and craving and relapse. While the reader of this chapter is provided with more information about the vulnerability of patients with co-occurring SUD and PTSD, the main focus here is on possible patient factors hindering the implementation of integrated SUD/PTSD treatment. Also, in this chapter a further insight is given into the perceived link between stress cues and craving/ relapse and trauma related triggers and craving/ relapse.

Building further on the perceptions of patients, in the subsequent chapter (6), results of a qualitative study are displayed. SUD/PTSD patients were interviewed about their first substance use and about their reasons for continuing their drugs or alcohol use. Furthermore, they were asked about their treatment preference. This chapter covers possible causes for the unsuccessful implementation of integrated SUD/PTSD treatment as does it cover preferences of the patient regarding treatment focus.

In the closing chapter, the primary results of the present dissertation will be summarized and its implications for clinical practice and further research are discussed. 


\title{
Chapter 2
}

\section{Prevalence of PTSD among patients}

\author{
with Substance Use Disorder
}

\section{Published as:}

Gielen, N., Havermans, R. C., Tekelenburg, M., \& Jansen, A. (2012). Prevalence of posttraumatic stress disorder among patients with substance use disorder: it is higher than clinicians think it is. European Journal of Psychotraumatology, 3. 


\section{Janis Joplin - What Good Can Drinkin' Do}

What good can drinkin' do, what good can drinkin' do?

Lord, I drink all night but the next day I still feel blue.

There's a glass on the table, they say it's gonna ease all my pain,

And there's a glass on the table, they say it's gonna ease all my pain.

But I drink it down, an' the next day I feel the same.

Gimme whiskey, gimme bourbon, give me gin.

Oh, gimme whiskey, give me bourbon, gimme gin.

'Cause it don't matter what I'm drinkin', Lord, as long as it

drown this sorrow I'm in. 


\begin{abstract}
This study had three objectives. Firstly, the prevalence of post-traumatic stress disorder (PTSD) and trauma exposure was compared between individuals with and without substance use disorder (SUD). Secondly, we compared self-rating of PTSD and clinical judgment. Thirdly, an analysis of the characteristics of SUD/PTSD patients was performed. The sample consisted of 423 patients with SUD and 206 healthy controls. All individuals were screened on PTSD using the self-rating inventory for PTSD. Significantly higher numbers of PTSD and trauma exposure were found in the SUD group (resp. 36.6 and 97.4\%). PTSD went frequently unnoticed when relying on clinical judgment alone. Patients with SUD/ PTSD were significantly more often unemployed and had a lower educational level. Axis I comorbidity and especially depressive disorders were more common in the SUD/PTSD group. It is concluded that patients with SUD/PTSD are a substantial and vulnerable subgroup in addiction treatment facilities and that a systematic screening for PTSD is required.
\end{abstract}




\section{Introduction}

Previous research has documented a strong link between substance use disorder (SUD) and posttraumatic stress disorder (PTSD). The prevalence of current PTSD (Diagnostic and Statistical Manual of Mental Disorders, Fourth Edition; DSM-IV) in SUD patients is around three times higher than in the general population, ranging from 25.3 to 49\% (Bonin, Norton, Asmundson, Dicurzio, \& Pidlubney, 2000; Driessen et al., 2008; Kessler et al., 2005; Ouimette, Coolhart, Funderburk, Wade, \& Brown, 2007; Read, Brown, \& Kahler, 2004; Reynolds et al., 2005). Besides this high comorbidity, studies suggest a poorer SUD treatment outcome when comorbid PTSD is left untreated (Mills, Teesson, Ross, Darke, \& Shanahan, 2005; Read et al., 2004).

Dragan and Lis-Turlejska (2007) used three different diagnostic tools to measure PTSD and found substantial differences in PTSD prevalence depending on which tool was used. Therefore, comparisons between prevalence studies are hard to make, unless they used the same measures in a similar way to assess the prevalence of PTSD. As of yet, no study has directly compared the prevalence of PTSD in a SUD population with the prevalence of PTSD in the general population using the same diagnostic tool within one and the same study.

In the present study three different aims can be distinguished: (1) to measure the prevalence of current PTSD and trauma exposure in a representative SUD sample relative to the prevalence of current PTSD and trauma exposure in the general population; (2) to assess whether a PTSD diagnosis is detected without using a standardised questionnaire; (3) to evaluate whether patients in the SUD/PTSD group are a more vulnerable subgroup in terms of socio-economic factors and psychiatric comorbidity.

In the present study the prevalence of PTSD was compared between a sample of individuals without substance dependence or abuse and a sample of treatment seeking SUD patients. To avoid confounding factors as symptoms of detoxification and craving and thereby the risk of overdiagnosis, we chose to measure PTSD with a validated symptom checklist [self-rating inventory for PTSD (SRIP); Hovens, Van der Ploeg, Bramsen, \& Klaarenbeek, 1994] extended with a trauma-exposure checklist. Within these scales, we measured PTSD symptoms that were prevalent in the past 4 weeks, thereby excluding lifetime and sub-threshold PTSD. Furthermore, we chose to explore PTSD prevalence in a representative SUD treatment seeking sample. In most prior prevalence studies only one specific substance group was included (Dragan \& Lis-Turlejska, 2007; Driessen et al., 2008; Johnson, 2008; Norman, Tate, Anderson, \& Brown, 2007). In clinical practice, however, polydrug use is very common and the same treatment protocol is applied to all patients, independent of their primary substance (Emmelkamp \& Vedel, 2007). To further improve the representativeness of our sample, no other 
exclusion criteria were imposed than being able to read and write (as they were expected to complete the SRIP).

A second objective of this study arose from conclusions of previous research, showing that PTSD often remains undiagnosed in SUD patients (Dansky, Roitzsch, Brady, \& Saladin, 1997; Najavits, 2005). This is important given that untreated PTSD may lead to a poorer outcome in SUD treatment (Back, Brady, Sonne, \& Verduin, 2006; Read et al., 2004). A diagnosis can be made by means of clinical judgement, by using standardised assessment instruments or by both means. It is interesting to find out whether PTSD is correctly diagnosed by clinicians without using a standardised questionnaire, based solely on their clinical experience. In the present study, we thus compared the prevalence of current PTSD as diagnosed by the clinician in the intake phase (without using a standardised diagnostic tool) with the prevalence of PTSD as diagnosed with the SRIP.

For clinical practice, it is interesting to examine whether SUD patients with PTSD are different from SUD patients without PTSD. If patients with SUD/PTSD have a lower socio-economic status or a more severe clinical profile, treatment of this vulnerable subgroup should have a more elaborated focus as well. Although most previous researchers agreed upon the more elaborated clinical comorbidity of SUD/PTSD patients (Bonin et al., 2000; Jacobsen, Southwick, \& Kosten, 2001; Mills, Lynskey, Teesson, Ross, \& Darke, 2005; Peirce, Kindbom, Waesche, Yuscavage, \& Brooner, 2008; Read et al., 2004), contradicting results were found with regard to socio-economic factors as employment, educational and relational status. With some researchers finding a more optimistic profile (e.g., being employed, having a higher educational level) than others (Bonin et al., 2000; Driessen et al., 2008; Mills, Lynskey, et al., 2005; Mills, Teesson, Ross, \& Peters, 2006; Peirce et al., 2008; Ouimette, Goodwin, \& Brown, 2006). Inconsistency was also found with regard to the number of previous treatments, with Bonin et al. (2000) finding no differences between SUD patients and SUD/PTSD patients and Driessen et al. (2008) and Jacobsen et al. (2001) concluding that SUD/PTSD patients had more previous admissions to addiction treatment facilities. Therefore, in this study, we also examined patient characteristics.

In general, we hypothesised (1) a greater prevalence of PTSD and trauma exposure in patients with SUD as compared to the prevalence in the healthy control group, (2) an underdiagnosis of PTSD when not using a standardised tool and (3) a more severe clinical profile and a lower socio-economic status in the SUD/PTSD compared with the SUD/no PTSD group.

\section{Methods}

Study design 
The present study comprises a cross-sectional design comparing a group of SUD patients and a group of healthy controls.

\section{Setting}

For this study, ethical approval was given by the Institutional Review Board of Maastricht University. All control participants signed a consent form prior to their participation.

Patients with SUD were recruited from 11 different addiction treatment branches from a single organisation (Mondriaan) all located within the same region (southern Limburg, with a population of approximately 620,000 inhabitants, located in the most southern tip of the Netherlands). Participants were included in the study from October 2008 to May 2009. Patients gave permission that the information in their patient file could be used for research purpose. Data on the SRIP and traumaexposure checklist of the patients included in this study were retrieved from their respective patient files. Since October 2008, the patients in treatment for SUD at the Mondriaan treatment center are structurally screened with the SRIP and trauma-exposure checklist. Note that the data on these questionnaires were obtained after intake and sometimes even after years of treatment. As such, this allowed us to compare the clinician's diagnosis of PTSD at intake (i.e., without input from a standardized tool as the SRIP) against the prevalence of PTSD based on SRIP scores.

\section{Participants}

Figure 1 shows a flowchart for the inclusion and exclusion of participants in the current study. The initial sample consisted of 432 patients with SUD (patient group) and 475 control participants (non-patient control group). We tried to match the control group as closely as possible with the patient group on the basis of age and gender. Participants from the non-patient group were contacted by e-mail. These individuals had previously indicated on the Maastricht University website to be interested in participating in research projects. From the 475 contacted participants, 67 e-mails were automatically returned because of technical problems with email addresses of the recipients, and 144 individuals chose not to participate. The remaining 264 participants in the non-patient group received a website address where they could log in and complete all necessary demographic questions, the SRIP and the trauma-exposure checklist. 


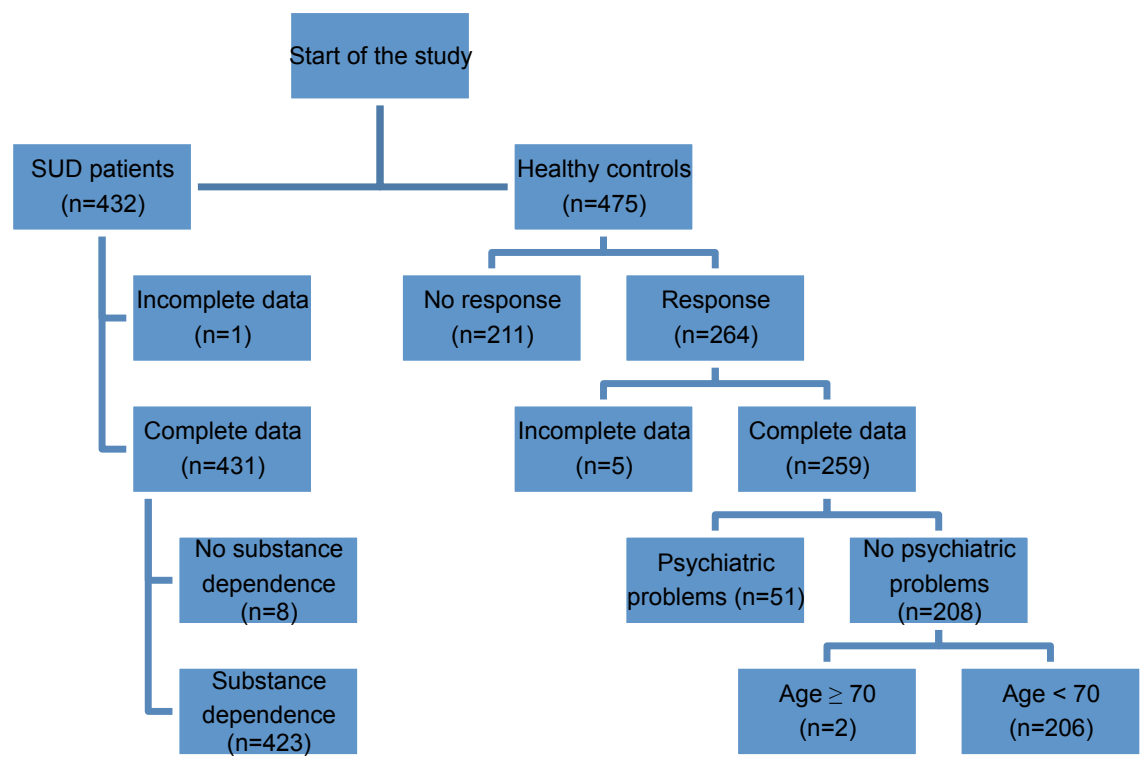

Figure 1. Flowchart inclusion participants.

Five persons from the non-patient group had incomplete data and were therefore excluded from further analyses. As we wanted to compare the prevalence of PTSD in SUD patients with that in healthy controls, we excluded a further 51 participants from the non-patient group because they admitted abusing drugs or alcohol or because of clear indications of other psychiatric problems. An indication for psychiatric problems was for instance the use of psychiatric medication. In the patient group only one individual was excluded from the analyses due to deficient data and eight patients were excluded because they did not fulfil DSM-IV-TR criteria for substance dependence. As we wanted to match groups on age as closely as possible, two more candidate participants (of 74 and 75 years of age) of the control group were excluded from further analysis. Participants in the patient group were between 18 and 68 years of age ( $M$ age $=41$ years), as opposed to an age range of 22-68 years in the non-patient group ( $M$ age $=54$ years). In the patient group 332 (78.5\%) participants were male and in the non-patient group 65\% was male. In total, data of 423 SUD patients and 206 control participants remained for further analyses. Participant characteristics for the total sample and the SUD patient sample (with and without PTSD) are displayed in Tables 1 and 2, respectively. 
Table 1. Total sample characteristics (SUD vs. Control).

\begin{tabular}{|c|c|c|c|c|c|}
\hline & $\begin{array}{l}\text { Total sample } \\
\text { (N=629) }\end{array}$ & $\begin{array}{l}\text { SUD } \\
(N=423)\end{array}$ & $\begin{array}{l}\text { Control } \\
(\mathrm{N}=206)\end{array}$ & Statistics & Significance \\
\hline \multicolumn{6}{|c|}{ Demographics } \\
\hline Mean age & 45.4 & 41 & 54.4 & $U=16117$ & $p<.001$ \\
\hline (SD) & $(12.64)$ & $(10.97)$ & $(10.96)$ & $r=-.51$ & \\
\hline \multicolumn{6}{|c|}{ Gender (\%) } \\
\hline Male & 74 & 78.5 & 65 & $\chi 2(1)=13.03$ & $p<.001$ \\
\hline Female & 26 & 21.5 & 35 & & \\
\hline \multicolumn{6}{|l|}{ Education } \\
\hline \multirow[t]{2}{*}{ Low } & 28.1 & 38.5 & 6.8 & $x^{2}(2)=$ & $p<.001$ \\
\hline & & & & 161.99 & \\
\hline Medium & 32.4 & 24.1 & 49.5 & & \\
\hline High & 16.7 & 4.3 & 42.2 & & \\
\hline Missing & 22.7 & 33.1 & 1.5 & & \\
\hline PTSD as & 28.6 & 36.9 & 11.7 & $\chi 2(1)=43.17$ & $p<.001$ \\
\hline \multicolumn{6}{|c|}{ measured by } \\
\hline the SRIP (\% & & & & & \\
\hline
\end{tabular}


Table 2. SUD patient sample characteristics (SUD/PTSD vs. SUD/no PTSD).

\begin{tabular}{|c|c|c|c|c|c|}
\hline & $\begin{array}{l}\text { SUD } \\
(N=423)\end{array}$ & $\begin{array}{l}\text { SUD/PTSD } \\
(\mathrm{N}=156)\end{array}$ & $\begin{array}{l}\text { SUD/no PTSD } \\
(\mathrm{N}=267)\end{array}$ & Statistics & Significance \\
\hline \multicolumn{6}{|c|}{ Demographics } \\
\hline Mean age & 41 & 41.6 & 40.6 & $U=19708$ & .390 \\
\hline (SD) & $(10.97)$ & (10.99) & (10.97) & $r=.04$ & \\
\hline \multicolumn{6}{|l|}{ Gender (\%) } \\
\hline Male & 78.5 & 75.6 & 80.1 & $\chi 2(1)=1.186$ & .276 \\
\hline Female & 21.5 & 24.4 & 19.9 & & \\
\hline \multicolumn{6}{|l|}{ Primary } \\
\hline \multicolumn{6}{|c|}{ substance (\%) } \\
\hline Alcohol & 43.7 & 46.2 & 42.3 & $x 2(4)=2.686$ & .612 \\
\hline Heroin & 33.6 & 33.3 & 33.7 & & \\
\hline Cocaine & 7.1 & 7.1 & 7.1 & & \\
\hline Cannabis & 10.9 & 7.7 & 12.7 & & \\
\hline Speed & 3.5 & 3.2 & 3.7 & & \\
\hline Missing & 1.2 & 2.6 & .4 & & \\
\hline
\end{tabular}

\section{Measurement/data sources}

\section{Self-rating inventory for PTSD}

In all participants PTSD was assessed using the SRIP. The SRIP is a Dutch self-report questionnaire with 22 items covering the 17 symptoms and the 3 symptom clusters (i.e., re-experiencing, persistent avoidance and increased arousal) of PTSD following the DSM-IV (Van Balkom, De Beurs, Hovens, \& Van Vliet, 2004). Note that the SRIP does not cover criteria A, E and F as formulated in the DSM-IV. Participants are asked to rate their symptoms over the previous 4 weeks on a 4 -point Likert scale $(1=$ not at all, 4 =extremely). Psychometric properties of the SRIP have proved to be good in different clinical samples, including trauma patients (Hovens, Bramsen, \& Van der Ploeg, 2002; Hovens et al., 1994). Cronbach's alpha reliabilities are 0.90-0.94 for the total SRIP score, 0.77-0.87, 0.80-0.88 and 0.76-0.85 
for the subscales intrusion, avoidance and hyperarousal, respectively. The questionnaire has an excellent test-retest reliability, with Pearson's $r=0.92$ for the total score and for the subscales intrusion, avoidance and hyperarousal the correlations are $0.84,0.91$ and 0.85 , respectively (Hovens et al., 2002). Sensitivity and specificity of the SRIP were measured with the clinician-administered PTSD Scale (CAPS) (Blake et al., 1995; Weathers, Keane \& Davidson, 2001), as an external criterion. Sensitivity (true positives according to the CAPS/true positives-false negatives) was high, ranging between 0.83 and 0.86 , and a good specificity (true negatives according to the CAPS/true negatives-false positives) ranging between 0.69 and 0.73 was found (Hovens et al., 2002; Witteveen, Bramsen, Hovens, \& Van der Ploeg, 2005). The high sensitivity reflects a low risk for false positives, i.e., a low risk for overdiagnosis. On the basis of the balance between sensitivity and specificity an optimum cut-off score for the diagnosis of PTSD of 52 was established (Hovens et al., 2002; Witteveen et al., 2005). Concurrent validity was also examined. Correlations with two other frequently used PTSD scales (Mississippi Combat-related PTSD Scale, civilian version and the Keane MMPI-PTSD subscale) are 0.80 and 0.82 , reflecting an adequate concurrent validity (Hovens et al., 2002).

\section{Trauma-exposure checklist}

To prevent the risk of overdiagnosis of PTSD, the SRIP, which is a symptom-only measure and does not cover criteria A of DSM-IV, was complemented with a trauma-exposure checklist. This checklist was based on different questionnaires and diagnostic interviews (Interview for Traumatic Events in Childhood; Lobbestael, Arntz, Harkema-Schouten \& Bernstein, 2009; Traumatic Experiences Questionnaire; Nijenhuis, Van der Hart, \& Kruger, 2002 and the Dutch translated version of the Structured Childhood Trauma Interview; Van der Bossche, Kremers, Sieswerda, \& Arntz, 1999) to include a broad range of possible traumas, such as a life-threatening situation, being attacked or threatened. In the present study, a positive diagnosis of PTSD was possible only when all of the following conditions were fulfilled: total SRIP is 52 or higher (Hovens et al., 2002), at least one intrusion item is $\geq 3$, three or more avoidance items are $\geq 3$, at least two hyperarousal items are $\geq 3$ ( $\mathrm{H}$. Hovens, personal communication, September 2008) and participants indicate on the trauma-exposure checklist to have been exposed to one or more traumatic events.

Mini International Neuropsychiatric Interview (MINI) 
To ensure that participants in the control group were not abusing drugs or alcohol, substance-related questions were added derived from the MINI (Sheehan et al., 1997). As participants in the patient group were all patients in addiction treatment SUD was not measured in this group.

\section{Statistical methods}

All analyses were conducted using SPSS 18.0 for Windows. The differences in PTSD prevalence and trauma-exposure prevalence between groups were analyzed by using chisquare tests. To compare different characteristics of SUD/PTSD patients with SUD/non-PTSD patients we used chi-square tests (for categorical variables) or $t$-tests for independent samples (for continuous variables). Cramer's $V$ is used to indicate the effect size for the results of the chi-square tests. The Mann-Whitney test was used for continuous data when the assumption of normality was violated. Logistical regression was used to test whether age and sex predicted PTSD.

\section{Results}

Prevalence of current PTSD and trauma exposure (SUD vs. Control)

In the sample of SUD patients, $36.6 \%$ scored positive on current PTSD. In the control group $10.2 \%$ could be diagnosed with PTSD. There was a significant relation between SUD and whether or not a person scored positive on PTSD $\chi 2(1)=48.09, p<0.001$, with Cramer's $V=0.28$, representing a small effect size. This reflects the fact that based on odds ratio individuals are 5.27 times more likely to score positive on PTSD if they are patients with SUD than if they are not patients with SUD.

Since patients with alcohol dependence and patients with heroin dependence constituted a large sample of the SUD patients (resp. 43.7 and 33.6\%), PTSD prevalence was also calculated for these subgroups. These numbers were comparable with PTSD prevalence in the SUD group (38.4 and 36.6\%, respectively).

Since age and sex were not equally distributed between the SUD group and the control group, we conducted another logistic regression analysis controlling for sex and age. The inclusion of sex and age in the model did not have any influence, nor were there any interaction effects. Only the group (SUD vs. healthy control) was a significant predictor for PTSD $(B=-1.632(.251), p<.001)$.

To analyze the continuous SRIP scores (with a higher score representing a more severe PTSD symptomatology), we employed a non-parametric test as the assumption of normal distribution was violated. Patients with SUD (median $=47$ ) had a significantly higher score on PTSD (total SRIP score) than 
control subjects (median =33), $U=18880.00, p<0.001, r=-0.44$. This again confirms the proposed strong association between SUD and PTSD.

In the control group $86.3 \%$ had been exposed to criterion A1 trauma. In the SUD group, $97.4 \%$ had encountered such a trauma in their lifetime. There was a significant relation between SUD and whether or not an individual had been exposed to trauma, $\chi 2(1)=28.89, p<0.001$, with Cramer's $V$ $=0.22$, representing a small effect size. On the basis of the odds ratio, individuals are 5.91 times more likely to have been exposed to trauma if they are SUD patients than if they are not SUD patients. Subjects were asked to indicate on a trauma-exposure checklist to which trauma they were exposed to in their lifetime. Table 3 displays the different types of exposed trauma as indicated by the subjects.

Table 3. Type of trauma (SUD vs. Control).

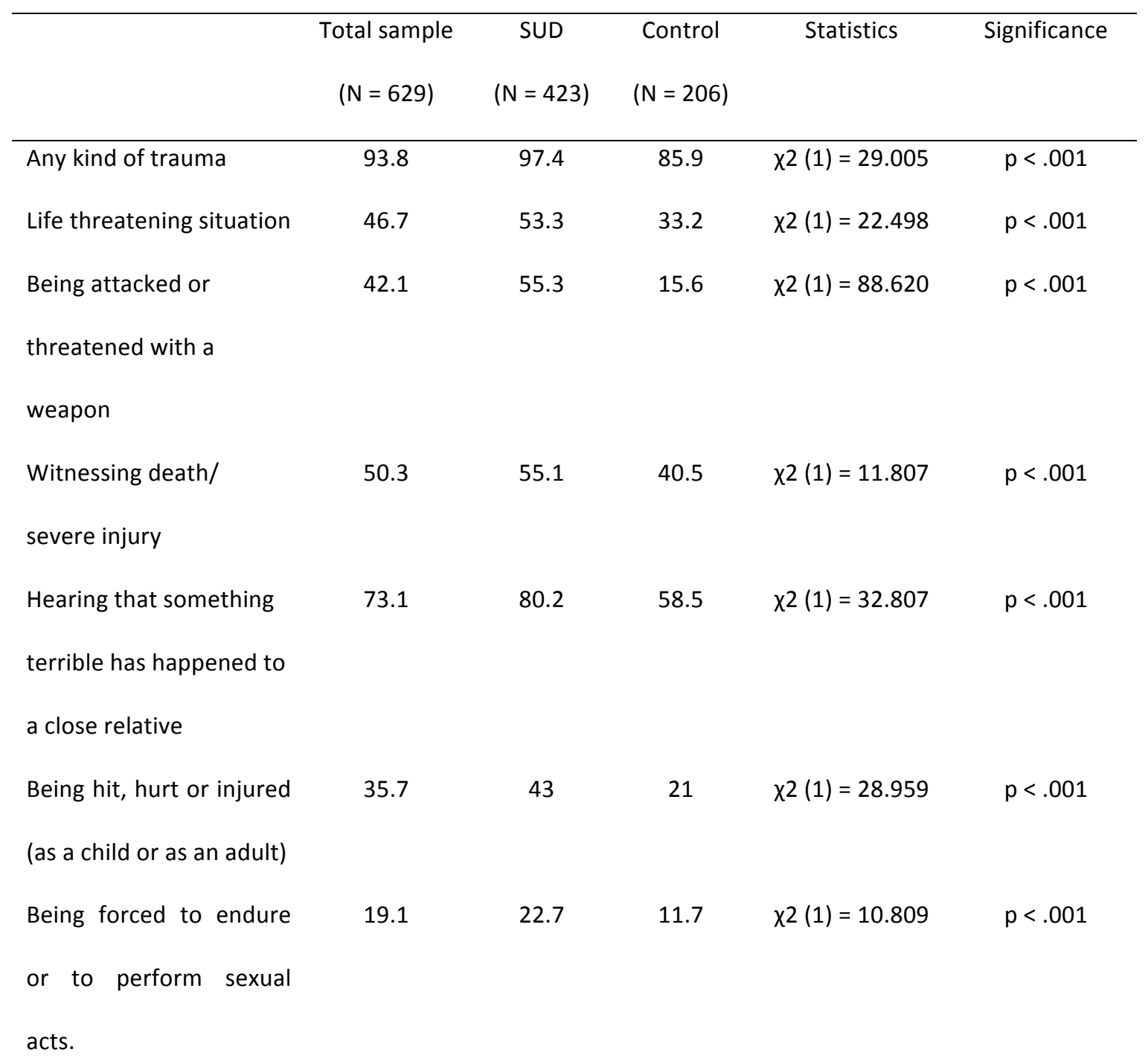


Relation between PTSD as diagnosed during intake and PTSD diagnosed with the SRIP

A total of $37.9 \%$ of patients with SUD mentioned a traumatic experience during the intake interview as reported in the corresponding patient files. A chi-square analysis was performed to check whether this was correlated with a positive PTSD diagnosis as assessed with the SRIP. There was a significant relation between trauma mentioned at intake and whether or not a person scored positive on PTSD as assessed with the SRIP, $\chi 2(1)=15.15, p<.005$, with Cramer's $V=0.19$, representing a small effect size.

In the anamnestic phase, one-fifth (20.2\%) of the patients had complained about one or more PTSD symptoms (e.g., recurrent nightmares, avoidance of traumatic stimuli, exaggerated startle response, etc.). There was a significant relation between PTSD symptoms mentioned at intake (mentioning vs. no mentioning) and whether or not a person scored positive on PTSD as assessed with the SRIP $\chi 2(1)=15.57, p<.001$, with Cramer's $V=0.19$, representing a small effect size.

In $4.8 \%$ of the intakes, possible PTSD was noted by the interviewer (mere clinical judgment, no use of questionnaires). After intake, a multi-disciplinary staff decides which diagnoses should be written in the patient files (again mere clinical judgment, no use of questionnaires). Only $2.1 \%(n=9)$ of the admitted SUD patients were actually diagnosed with PTSD. When comparing this result with the number of PTSD diagnoses as measured with the SRIP (36.6\%), it is clear that a large discrepancy in PTSD diagnosis exists, depending on whether or not a questionnaire is used. In four of these nine patients where PTSD was diagnosed type II error seemed to be prevalent given that the clinical judgment diagnosis was not confirmed by the SRIP.

Clinical profile and socio-economic status of SUD patients with vs. without PTSD

We derived information on clinical profile and socioeconomic status of the patients from their respective patient files. The SUD/PTSD patients appeared to be quite similar to the SUD/no PTSD patients. No significant differences were found with regard to the criminal past [ $\chi 2(1)=0.95, p=0.35$ ], financial debts $[\chi 2(1)=3.19, p=0.08]$ and relationship status. Both groups were comparable regarding their time in treatment $[\chi 2(3)=1.75, p=0.63]$, number of previous treatments [ $\chi 2(3)=3.78, p=0.29$ ] and treatment outcome $[\chi 2(1)=0.80, p=0.67]$. Two demographic factors were markedly different between the two groups: education level $[\chi 2(2)=14.38, p<0.01]$ and current employment status [X2 (1) $=4.05, p<0.05]$. SUD/PTSD patients were significantly more often unemployed and had a lower educational level. 
We further examined whether there might be a more severe pattern of comorbidity (axis I or II) in patients with SUD/PTSD. A significant relation existed between depressive disorders and PTSD. Patients with SUD/PTSD were more likely to have depressive disorders [X2 $(2)=13.32, p<0.001$, with Cramer's $V=0.19$ ] than SUD/no PTSD patients. Secondly, SUD/PTSD patients were significantly more likely to be patients with other axis I disorders compared to SUD/no PTSD patients $[\chi 2(1)=13.78, p$ $<0.001$, with Cramer's $V=0.19]$. No other significant results were found in examining axes I and II disorders in both groups.

\section{Discussion}

A first goal of the study was to gain insight into the prevalence of PTSD and trauma exposure in SUD patients and to compare these numbers with the prevalence of current PTSD and trauma exposure in a healthy control group using the same diagnostic tool. In the present study, we found that $36.6 \%$ of SUD patients scored positive on PTSD. Almost every patient had encountered at least one traumatic experience in his/her lifetime. As hypothesized, these numbers were significantly lower in individuals without substance dependence. In this group we found a PTSD prevalence rate of $10.2 \%$, and $86.3 \%$ of the persons in this control group had experienced some kind of traumatic event.

We cannot directly compare these results with earlier research, because this study is the first, to our knowledge, that contrasts the prevalence of current PTSD in a heterogeneous SUD population with PTSD in a control group within the same study using the same questionnaire. Nevertheless, the results of some comparable studies (measuring current PTSD in a heterogeneous SUD population) are consistent with our prevalence data. In prior studies in SUD populations, current PTSD was found to be prevalent in 25.3 - 41\% of cases (Bonin et al., 2000; Driessen et al., 2008; Ouimette et al., 2007; Read et al., 2004; Reynolds et al., 2005). Studies in SUD populations that assessed prevalence rates of trauma exposure typically report percentages between 89 and 95\% (Dansky, Saladin, Coffey, \& Brady, 1997; Farley, Golding, Young, Mulligan, \& Minkoff, 2004; Read et al., 2004; Reynolds et al., 2005), which concurs with the present results.

One discrepant finding with previous research is the relatively high number of current PTSD in the control group; in this study, $10.2 \%$ of the control subjects had a positive diagnosis. It should be noted that the present sample of healthy controls does not represent the general population. These healthy controls signed up as voluntary participants for psychology research in response to a recruitment advertisement on the university website. Conceivably, individuals who suffer(ed) from psychological problems may be more readily inclined to participate in psychological research such as the 
present study. Indeed, other researchers have found a typical prevalence rate below $4 \%$ in the general population (Bromner, Peek, Bronner, Last, \& Grootenhuis, 2009; Creamer, Burgess \& McFarlane, 2001; Darves-Bornoz et al., 2008; De Vries \& Olff, 2009). Another possible explanation for the high number of PTSD in the control group might be that the SRIP overestimates PTSD. This option does not seem valid because of two reasons. Firstly, researchers documented a good specificity for the SRIP, reflecting a low risk for overdiagnosis (Hovens et al., 2002; Witteveen et al., 2005). Secondly, if the SRIP overestimates PTSD, the same profile should be noted in the SUD group.

The fact that many SUD patients score positive on PTSD has important implications for clinical practice. At present, PTSD receives little attention in SUD treatment (Najavits, Sullivan, Schmitz, Weiss, \& Lee, 2004) and systematically conducted screening with a validated questionnaire does not take place in more than $80 \%$ of SUD patients (Young, Rosen, \& Finney, 2005). This study emphasizes the need to identify the SUD/PTSD patients as a substantial and important subgroup and to systematically screen for PTSD in all SUD patients at intake.

Our second aim was to assess whether PTSD would be correctly diagnosed by interviewers and clinicians without using a standardized questionnaire. As expected, PTSD remained largely undetected when no standardized tool was used. These findings are important because they draw attention to the fact that, with regard to the diagnosis of PTSD, SUD treatment facilities should not solely rely on the opinions of clinicians and interviewers. Clinical judgement is prone to bias and, when possible, validated assessment tools should be used (Dawes, Faust, \& Meehl, 1989; Garb, 2005).

The third and final goal was to evaluate whether patients in the SUD/PTSD group have a more severe clinical profile and a lower socio-economic status. Somewhat surprisingly, the SUD/PTSD patients and the group of SUD/no PTSD patients proved comparable on most patient characteristics. There were, however, four significant differences between the groups: (1) patients in the SUD/PTSD group were more often less educated, (2) the SUD/PTSD comorbid patients were more often unemployed, (3) patients with PTSD were more likely to have depressive disorders and (4) patients with PTSD were more likely to have another axis I disorder.

In sum, the present study provides further evidence for the heightened prevalence rate of PTSD among SUD patients. The treatment facilities participating in this research did not employ structural testing for PTSD among newly admitted patients. Clearly, this is associated with severe underdiagnosis of PTSD. This is a problem as it has been shown that SUD patients with comorbid PTSD may benefit from parallel treatment of their PTSD (Amaro et al., 2007; Cocozza et al., 2005; Coffey, Stasiewicz, Hughes, \& Brimo, 2006; Morrissey et al., 2005; Najavits, Gallop, \& Weiss, 2006; Van Dam, Vedel, Ehring, \& 
Emmelkamp, 2012), and patients are denied a potentially more favourable treatment outcome when their PTSD remains undiagnosed (Mills, Teesson, et al., 2005; Read et al., 2004). 


\section{Chapter 3}

\section{Craving after trauma and stress cue exposure}

Gielen, N., Nederkoorn, C., Jansen, A., \& Havermans, R.C. (submitted for publication). Cued cravings in substance dependent patients with posttraumatic stress disorder. 


\section{Eminem - going-through-changes}

And-it-hurts-so-i-fast-forward-sleeping-pills-make-me-feelalright-

and-if-im-still-awake-in-the-middle-of-the-night

I-just-take-a-couple-more-yeah-youre-motherfuckin-right- 


\begin{abstract}
Previous research shows that patients with substance use disorder (SUD) and post-traumatic stress disorder (PTSD) experience more craving for a substance after exposure to their personal trauma cues compared to neutral cues. However, it is not clear if their substance cravings are triggered by specifically trauma cues or by stress cues in general and whether the level of PTSD matters. The current study examined the impact of trauma cues and stress cues on substance craving and tension in patients with SUD and different levels of PTSD severity. Seventy-four patients attended three exposure sessions in which the patient was exposed to one tape recorded script; a neutral script, a stress script and a trauma script. Craving and tension were measured before and after each cue exposure. The findings show that patients high in PTSD severity craved more after stress and trauma script exposure, compared to exposure to the neutral script and to patients low in PTSD severity. These findings suggest that SUD/PTSD patients are not only more vulnerable to trauma cues, but also to more general stress cues. Treatment implications are discussed.
\end{abstract}




\section{Introduction}

Substance Use Disorder (SUD) and Post Traumatic Stress Disorder (PTSD) frequently co-occur. Previous studies demonstrate PTSD prevalence rates of around 35\% in SUD patients (Driessen et al., 2008; Gielen, Havermans, Tekelenburg, \& Jansen, 2012; Read, Brown, \& Kahler, 2004). This rate is considerably higher than in the general population, where the lifetime prevalence of PTSD is between $6.8 \%$ and $9.2 \%$ (NCS-R lifetime prevalence rates; Keane, Marshall, \& Taft, 2006).

Experts generally believe that substance use of SUD/PTSD patients regulates the negative emotions associated with PTSD (Simpson \& Miller, 2002; Stewart, Pihl, Conrod, \& Dongier, 1998). According to this so-called self-medication hypothesis, trauma exposure should precede, rather than follow, the development of SUD, and this is exactly what has been found in a recent longitudinal study (Haller \& Chassin, 2014). Furthermore, it is documented that a positive diagnosis of PTSD puts individuals at risk for the onset of drug abuse or dependence (Breslau, Davis, \& Schultz, 2003; Reed, Anthony, \& Breslau, 2007, Simpson \& Miller, 2002). The self-medication hypothesis on PTSD in the development of SUD predicts that the substance use itself should alleviate PTSD symptoms. Indeed, Stewart et al. (1998) argue that drugs with arousal-dampening properties (e.g., alcohol) are used to reduce the hypervigilance toward trauma related cues.

SUD patients with comorbid PTSD are found to be more vulnerable for relapse when they are confronted with interpersonal negative emotions, as compared to patients with SUD only (Ouimette, Coolhart, Funderbuck, Wade, \& Brown, 2007). Other studies indicate that SUD/PTSD patients, compared to those who have SUD only, use more alcohol and drugs, were more often in treatment, receive more treatment sessions and experience higher levels of craving, distress and psychiatric symptoms (Back, Sonne, Killeen, Dansky, \& Brady, 2003; Dube, Anda, Feliti, Chapman, Williamson, \& Giles, 2001; Evren, C., Evren, B., Dalbudak, Ozcelik, \& Oncu, 2009; Gielen et al., 2012; Mills, Lynskey, Teesson, Ross, \& Darke, 2005; Ouimette, Brown, \& Najavits, 1998; Pirard, Sharon, Kang, Angarita, \& Gastfriend, 2005; Read et al., 2004; Simpson \& Miller, 2002). Furthermore, some recent reviews report evidence for the effectiveness of integrated SUD-PTSD treatment (Berenz \& Coffey, 2012; Fareed, Eilender, Haber, Bremner, Whitfield, \& Drexler, 2013; McCauley, Killeen, Gros, Brady, \& Back, 2012; van Dam, Vedel, Ehring, \& Emmelkamp, 2012).

If SUD-patients with PTSD indeed misuse substances specifically to escape or suppress the stress evoked by trauma memories, such memories should be strongly associated with drug use. In that case, the trauma memories might function as conditioned drug stimuli that, due to their association with drug use (and hence a drug effect), easily come to elicit strong cravings. Indeed, it was repeatedly found that 
patients with PTSD and alcohol or cocaine dependency experience more desire for substance use after exposure to personalized trauma cues (Coffey, Saladin, Drobes, Brady, Dansky, \& Kilpatrick, 2002; Coffey, Schumacher, Stasiewicz, Henslee, Baillie, \& Landy, 2010; Nosen, Nillni, Berenz, Schumacher, Stasiewicz, \& Coffey, 2012; Saladin, Drobes, Coffey, Dansky, Brady, \& Kilpatrick, 2003). In 2002, Coffey et al. were the first to examine the impact of trauma imagery cues together with in vivo drug cues on cued cravings in patients with SUD and PTSD. They compared alcohol dependent PTSD individuals and PTSD patients with cocaine dependency. Both groups displayed cue reactivity (i.e., increased cravings) following simultaneous exposure to the personalized trauma cues and drug cues. Saladin et al. (2003) exposed alcohol and cocaine dependent patients with and without PTSD to four pairs of cues (trauma + drug, trauma + neutral, neutral + drug and neutral + neutral). The trauma scripts and the drug cues elicited significantly higher levels of craving compared to the corresponding neutral script/ cue. PTSD severity predicted the degree of craving after the trauma script exposure, regardless of the presence of substance cues. Not only self-reported craving increased after trauma cue exposure, also physiological cue reactivity (salivary flow) was demonstrated (Coffey et al., 2010). Moreover, decreased drug cue reactivity was found in SUD/PTSD patients after some imaginary trauma exposure treatment sessions (Coffey, Stasiewicz, Hughes, \& Brimo, 2006; Nosen, Littlefield, Schumacher, Stasiewicz, \& Coffey, 2014).

Although these pioneering cue-reactivity studies clearly show increased drug cravings during exposure to trauma cues, the studies did not take stress cue reactivity into account. In a recent study we found that SUD/PTSD patients show significantly less efficient coping strategies as compared to SUD patients with less PTSD symptoms (Gielen, Nederkoorn, \& Havermans, 2015a), and recent neurobiological studies show a disturbed stress reactivity response in patients trauma exposure (Klaassens, Giltay, Cuijpers, van Veen, \& Zitman, 2012; Vermetten \& Lanius, 2012). Therefore, besides testing the link between craving and trauma cues, it might be of interest to test how SUD/PTSD patients react to personalized stress scripts, unrelated to their traumas. If cued cravings are not specific to trauma and drug cues but related to more general stressors this might have implications for treatment, e.g., then treatment might benefit from also including the learning of coping skills. In the present study, we tested whether cued cravings of SUD+PTSD patients are specific for trauma cues or also more general to stress cues. It is hypothesized that patients with high scores on PTSD severity will experience more subjective cravings in response to stress cues and trauma cues than subjects with less PTSD symptoms. We further expect higher cravings in response to trauma cues compared to stress cues and higher cravings in response to stress cues compared to neutral cues. 
SUD patients with a history of trauma exposure and with different levels of PTSD severity will be exposed to neutral cues, stress cues and trauma cues. Craving and tension will be assessed before and immediately after cue exposure.

\section{Method}

\section{Participants}

All substance dependent individuals engaging in one of six wards of an intramural substance abuse treatment program that does not specifically address PTSD or trauma were informed about the study and invited to participate. The patient information letter and informed consent were explicated. If the patient agreed to participate in the study, inclusion and exclusion criteria were checked. Main inclusion criteria for the current study were being exposed to a past trauma (based on the Trauma-Exposure Checklist, descript below) and being treated clinically for SUD. To avoid influences of detoxification or recent substance use on craving we only allowed patients who were in clinical treatment for at least 20 days. Although complete abstinence was expected for these patients, relapse during clinical treatment is not uncommon. Individuals whose last lab session could not be planned 2 days before their inpatient discharge or earlier were not allowed to participate in the study. Participants were ineligible if they met criteria for a psychotic disorder, when they had a verbal IQ lower than 80 or when they were at risk for suicidality or self-injurious behavior. Illiterate patients or patients who did not speak and understand the Dutch language could not take part in the study. Patients who were taking medications that prohibited craving (e.g. naltrexone, acamprosate, methadone) were asked to sustain to the same dose during the study period (with permission of the attending psychiatrist).

In total, 91 SUD patients with a trauma history were included in the study. The study was conducted with the approval of the medical ethical committee of Atrium MC, Orbis MC, and Hogeschool Zuyd in Heerlen, the Netherlands. All participants provided written informed consents and were paid 20 $€$ after study completion. Since 17 patients did not complete the full study, the data of 74 patients were analyzed.

\section{Materials}

\section{Self-rating Inventory for Posttraumatic Stress Disorder (SRIP)}

PTSD symptoms were diagnosed with the validated Dutch self-rating inventory for PTSD (SRIP; Hovens, Van der Ploeg, Bramsen, Klaarenbeek, Schreuder, \& Rivero, 1994). The SRIP is a questionnaire with 22 items covering the 17 symptoms and the 3 subscales (i.e. reexperiencing, avoidance and hyperarousal) 
of PTSD following the DSM-IV (van Balkom, de Beurs, Hovens, \& van Vliet, 2004). Participants rated their symptoms over the previous 4 weeks on a 4 -point Likert scale $(1=$ not at all, $4=$ extremely). Psychometric properties of the SRIP have proven to be good in different clinical samples, including PTSD patients (Hovens et al., 1994; Hovens, Bramsen, \& Van der Ploeg, 2002; Witteveen, Bramsen, Hovens, \& Van der Ploeg, 2005) and SUD/PTSD patients (Kok, de Haan, van der Velden, van der Meer, Najavits, \& de Jong, 2013). A positive PTSD diagnosis requires a SRIP-total score of 52 or higher (Hovens et al., 2002; Witteveen et al., 2005), at least one intrusion item scored $\geq 3,3$ or more avoidance items scored $\geq 3$, and at least 2 hyperarousal items scored $\geq 3$ (H. Hovens, personal communication, September 2008).

\section{Trauma-exposure checklist}

This checklist is based on different questionnaires and diagnostic interviews (Interview for Traumatic Events in Childhood; Lobbestael, Arntz, Harkema-Schouten, \& Bernstein, 2009; Traumatic Experiences Questionnaire; Nijenhuis, van der Hart, \& Kruger, 2002 and the Dutch translated version of the Structured Childhood Trauma Interview; Bossche, Kremers, Sieswerda, \& Arntz, 1999) to include a broad range of possible traumas, such as a life threatening situation, being attacked or threatened.

\section{Imagery cues}

Trauma cues. Personalized script driven imagery is a well-validated and reliable procedure across PTSD populations to induce responses that are similar to what happens when a PTSD patient is confronted with a traumatic trigger. It has been shown to elicit stronger psychophysiological and mood responses than generic scripts (Coffey et al., 2002; 2010; Saladin et al., 2003; Sinha, 1996). Studies have shown that personalized scripts are also effective for inducing craving in patients with comorbid SUD/PTSD (Kwako, Schwandt, Sells, Ramchandani, Hommer, George et al., 2014; Nosen et al., 2014). Stress cues. Personalized scripts were also used as general stressor stimuli. The participants first listened to a standard instruction text regarding the type of traumatic or stressful event that the study aimed for. This was important since participants had to be able to distinguish between what counts as a trauma and what counts as a stressful event. Traumatic events included experiences in which the person was confronted with or witnessed actual or threatened death, severe injury or events that threatened the physical integrity of self or others (4th ed., text rev.; Diagnostic and Statistical Manual of mental disorders; American Psychiatric Association [APA], 2000). Stressful events were described as events that were often associated with family, friends, partner, work or money and in which the person felt sad, angry or tensed. For both cues, the participants were instructed not to choose situations in which 
substance use played a major role. They were asked to think about events (resp. trauma and stress) that were most traumatic/ stressful to them. Participants rated the impact of the events on a $10 \mathrm{~cm}$ Visual Analogue Scale (VAS) $(0=$ not at all stressful/ traumatic and $10=$ the most stress I have felt in my life/ the most severe trauma l experienced). Only situations rated as 8 or above were accepted as appropriate for script development. The participants were then interviewed about each event and they were encouraged to include multiple sensory dimensions in their descriptions. The interviews were audiotaped to facilitate the script-making after the session. For each subject scripts were then developed portraying the subject's own experience in the second person, present tense. The personalized scripts had an approximate duration of $85 \mathrm{~s}$ and were audiotaped.

Neutral cues. Three neutral situations were used: (1) sitting on the beach and looking around, (2) sitting in the park, near the river and looking around and, (3) taking a bath. Participants rated these neutral situations using $10 \mathrm{~cm}$ VASs ranging from 0 'not at all' to 10 'very much': "In your case, is there a connection between this situation and substance use?", "In your case, is there a connection between this situation and stress?", "Can you imagine yourself in this situation?". For each participant, the most neutral and most imaginable situation was selected as neutral cue, but only when no association with substance use was reported.

\section{Craving and tension}

Craving and tension were rated on two separate $10 \mathrm{~cm}$ VASs, ranging from $\mathbf{0}$ (no craving/tension at all) to 10 (a lot of craving/tension).

\section{Manipulation checks: Imagery vividness and intensity of scripts}

Participants rated how vividly they were able to imagine the scenario on a $10 \mathrm{~cm}$ VAS ranging from 0 (not at all clear) to 10 (perfectly).

Three independent reviewers rated the stress and trauma scripts on intensity ("How intense do you experience the emotions elicited by this described event?") on a $10 \mathrm{~cm}$ VAS ranging from 0 (not intense at all) to 10 (very intense). This rating was included to ensure that the scripts were comparable with regard to intensity level.

\section{Procedure}

The experiment included four sessions for each participant; one assessment session and three lab sessions in which the cue exposure took place. 
In the assessment session, some information about the procedure was given and the participant filled out the trauma exposure checklist to ensure that there was a past trauma encounter. Next, the script development for the neutral cue occurred. At the end of this assessment session a short overview was given about the next session, and the participant was informed that it was important for the study to stay abstinent from drugs or alcohol. Finally, an appointment was made for the lab sessions. In total, the assessment session lasted around 20 minutes.

The 3 lab sessions had a similar structure and were planned for 3 successive weeks. At the beginning of each lab session the participants were informed that the researcher was obliged to follow an outlined protocol which prevented him/ her to go into possibly emotional issues. Nevertheless, at the end of each session there was ample opportunity to evaluate the session and to ventilate experiences. Next, compliance with the abstinence restrictions was assessed (self-report) and the participant was inquired about unusual stress or cravings, and about possible changes in medication dosage. Hereafter, the participant rated current tension (baseline tension) and current craving (baseline craving). (S)he then put the headphone on and listened with closed or averted eyes to the recorded text (neutral, trauma or stress). Neutral cue exposure was always planned in the first lab session, while the two other cues were counterbalanced for lab session 2 and 3 . The participant was instructed to imagine the script as vividly as possible. After the imaginary, tension and craving were rated again. Subsequently, the vividness of the imagination was rated. The participant was debriefed, focusing on personal strengths and available sources of support. Final tension and craving ratings were obtained to assure the safety of each participant upon dismissal. If participants' tension and craving were still above baseline levels a relaxation procedure was suggested. Emotion regulating strategies were offered until tension and craving dropped to baseline levels. In 7 persons the tension/craving remained higher than baseline, even after the emotion regulating strategies. In these cases, the therapist involved was informed about the increased tension/craving and these participants received special attention from their therapist after the session. All of these 7 participants completed the study and none of them relapsed during the study.

There were interviews added to the end of each lab session, after the study protocol and measures were completed. In the first lab session the script development for the trauma and the stress cue took place, and it included a structured interview about the patient's perceptions regarding the link between SUD and PTSD (published in Gielen et al., 2015a). The second and third session both included the assessment of a clinical interview on psychiatric comorbidity (published in Gielen et al., 2015a). In between the sessions, the patients filled out some self-report questionnaires (published in Gielen et al., 
2015a). At the end of the $3^{\text {rd }}$ lab session, participants were paid and they were thanked for their participation.

\section{Data analysis}

All statistical analyses are performed using SPSS version 21 software. A 2 (time of measurement: pre and post cue exposure) $\times 3$ (type of cue: neutral, stress or trauma) repeated measures ANCOVA was used to examine differences on the dependent measures (subjective craving and tension), with PTSD severity as covariate. For the two ANCOVAs, a Bonferroni adjustment was employed to reduce type I error.

To assess the impact of participants' imageability (vividness of the imagery) and the impact of the intensity of the scripts, Pearson correlation analyses were employed to test for relationships between imageability and intensity with cue reactivity.

Although data of 74 patients were used in this study, sample size varies depending on the completion of answers on different measures. Originally, subjective tension was only measured before cue exposure. We adapted the protocol and started measuring tension (post cue exposure) after the participation of the first 20 patients, so fewer patients are included in the analyses on tension.

\section{Results}

\section{Participant characteristics}

Participants were male (77\%) and female participants with a mean age of 39.45 years $(S D=9.14)$. They were in clinical treatment for the substance dependency only $(47.3 \%)$, for dual diagnosis (27\%) or for SUD + forensic issues (25.7\%). The SUD patients were exposed to different kinds of traumata during their past (mostly to multiple traumas). Physical abuse was most common (75.7\%), followed by exposure to life threatening situations (46.6\%) and sexual abuse (39.2\%). A minority was traumatized in war (8.1\%). Trauma exposure happened mostly at a younger age than first use of the primary substance $(\mathrm{M}=$ 13.27 [SD $=7.19]$ vs $M=22.87$ [SD = 8.49]). Only 8 patients $(11.1 \%)$ experienced their first trauma after the age of first use of the primary substance. Mean SRIP score was 50.49 (SD =11.54), which is around the 52 cut-off score for PTSD which was defined by Hovens and colleagues (2002). Alcohol was the primary substance for most participants $(54.1 \%)$ and mean abstinence was 13.73 weeks (SD = 19.77, range: 1 - 104). Note that thirty five percent of the sample stated that they (almost) never craved in the last month.

Other SUD characteristics of the sample are shown in Table 1. 
Table 1. SUD characteristics.

\begin{tabular}{ll}
\hline SUD characteristics $(\mathbf{n}=\mathbf{7 4})$ & \\
\hline Primary substance (\%) & \\
Alcohol & 54.1 \\
Benzodiazepines & 1.4 \\
Heroin & 13.5 \\
Cocaine & 14.9 \\
Cannabis & 10.8 \\
Speed (amphetamines) & 2.7 \\
Polydrug use & 32.4 \\
\hline (almost) all the time & 2.7 \\
\hline Craving last month (\%) & \\
\hline
\end{tabular}

\section{Manipulation checks}

Participants rated the vividness of each script after imagery. This imageability of the scripts differed significantly between the three conditions, $F(1.83,132.06)=7.43, p=.001$, contrast tests showed that stress and trauma cue imageability differed significantly from neutral cue imageability (resp. $F(1,72)=$ $10.15, p=.002$, and $F(1,72)=9.25, p=.003$ ) while stress and trauma cue imageability did not differ significantly from each other $(F(1,72)=.009, p=.92$.

Three independent reviewers rated the intensity of each script. The intensity differed significantly between conditions $F(1.73,119,60)=5448,09, p<.001$. Contrasts showed highly significant 
differences between neutral and stress cue intensity $(F(1,69)=7478.73, p<.001)$, between neutral and trauma cue intensity $(F(1,69)=13239.10, p<.001)$, and between stress and trauma cue intensity $(F(1$, $69)=94.33, p<.001)$. Table 2 displays the means for vividness of imagery and rated intensity.

Table 2. Manipulation checks of rated vividness of imagery and intensity of scripts.

\begin{tabular}{lcc}
\hline Mean imagination (SD) & $\mathrm{n}$ \\
\hline Neutral cue & $7.73(2.41)$ & 73 \\
Stress cue & $8.67(1.70)$ & 74 \\
Trauma cue & $8.65(1.82)$ & \\
\hline Mean intensity ratings (SD) & & 3 \\
\hline Neutral cue & $0.10(.10)$ & 3 \\
Stress cue & $7.26(.69)$ & 3 \\
Trauma cue & $8.19(.62)$ & \\
\end{tabular}

\section{Subjective tension}

Baseline tension

There was a significant relationship between the baseline measures of tension in the stress and trauma condition and PTSD severity (stress cue exposure: $r=.31, p=.01$; trauma cue exposure: $r=.30, p=.01$ ). There was no significant correlation between tension before neutral cue exposure and PTSD severity $(r=$ $.21, p=.07)$.

\section{Effect cue exposure on tension}

The 2 (time of measurement) $\times 3$ (type of cue) repeated measures ANCOVA with PTSD severity as covariate, showed no main effects for type of cue or time of measurement on tension. We did find a significant main effect of PTSD severity on tension, $F(1,55)=11.01, p=.002$, indicating that patients scoring high on PTSD in general experienced significantly more tension. There were no other significant effects. Table 3 displays the means and standard deviations of the tension ratings. 
Table 3. Subjective tension.

\begin{tabular}{llllll}
\hline Neutral Baseline & After neutral & Stress Baseline & After stress cue & Trauma Baseline & $\begin{array}{l}\text { After trauma } \\
\text { cue }\end{array}$ \\
& cue & & & & cue \\
$3.14(2.68)$ & $1.52(1.87)$ & $2.11(2.32)$ & $4.36(3.11)$ & $2.48(2.60)$ & $5.45(2.97)$ \\
$\mathrm{n}=74$ & $\mathrm{n}=58$ & $\mathrm{n}=74$ & $\mathrm{n}=59$ & $\mathrm{n}=74$ & $\mathrm{n}=59$ \\
\hline
\end{tabular}

Subjective craving

Table 4. Subjectively experienced craving before and after each cue (neutral, stress, and trauma; $n=74$ ).

\begin{tabular}{llllll}
\hline Neutral Baseline & After neutral & Stress Baseline & After stress cue & Trauma Baseline & After trauma \\
$M(S D)$ & cue & & $M(S D)$ & $M(S D)$ & cue \\
& $M(S D)$ & $M(S D)$ & & & $M(S D)$ \\
$.97(1.81)$ & $.69(1.49)$ & $.74(1.58)$ & $1.29(2.14)$ & $.79(1.74)$ & $1.79(2.59)$ \\
\hline
\end{tabular}

\section{Baseline craving}

Table 4 displays all craving ratings. There was a significant relationship between all three baseline measures of craving and PTSD severity, indicating that patients with higher severity of PTSD craved more in general (neutral cue exposure: $r=.29, p=.01$; stress cue exposure: $r=.27, p=.02$; trauma cue exposure: $r=.38, p \leq .001)$.

\section{Effect cue exposure on craving}

The 2 (time of measurement) $\times 3$ (type of cue) repeated measures ANCOVA with PTSD severity as covariate showed no significant main effects for type of cue or time of measurement on craving (resp. $F(2,71)=1.54, p=.22$, and $F(1,72)=.53, p=.47)$. There was, however, a significant main effect of PTSD severity on craving, $F(1,72)=15.11, p<.001$, indicating that patients scoring high on PTSD in general craved significantly more. There was also a significant three-way interaction effect between type of cue (neutral, stress, trauma), time of measurement (before vs. after cue exposure) and PTSD severity, $F=$ 3.66, $p=.03$. This indicates that PTSD severity had different effects on craving depending on which cue was presented and whether craving was measured before or after cue exposure. To break down this interaction effect, contrasts were performed comparing stress and trauma cue reactivity with neutral cue reactivity and comparing craving after and before cue exposure. These revealed significant interactions between time and cue type when stress cue reactivity is compared to neutral cue reactivity 
$(F(1,72)=5.38, p=.02)$ and when trauma cue reactivity is compared to neutral cue reactivity $(F(1,72)=$ $4.35, p=.04)$ : after stress and trauma exposure, craving is higher than after neutral exposure. Finally, further analyses showed that there was no significant interaction between PTSD severity and time of measurement when patients were exposed to the neutral script $(F(1,72)=1.81, p=.18)$, but that this interaction effect was significant for the stress script $(F(1,72)=5.20, p=.02)$ and for the trauma script $(F(1,72)=4.14, p=.04)$, indicating that patients with high PTSD severity craved more after both stress and trauma exposure compared to neutral exposure.

To visualize the craving responses we performed a median split based on PTSD severity (median =49.5), showing patients scoring lower and higher on PTSD (see Figure 1).

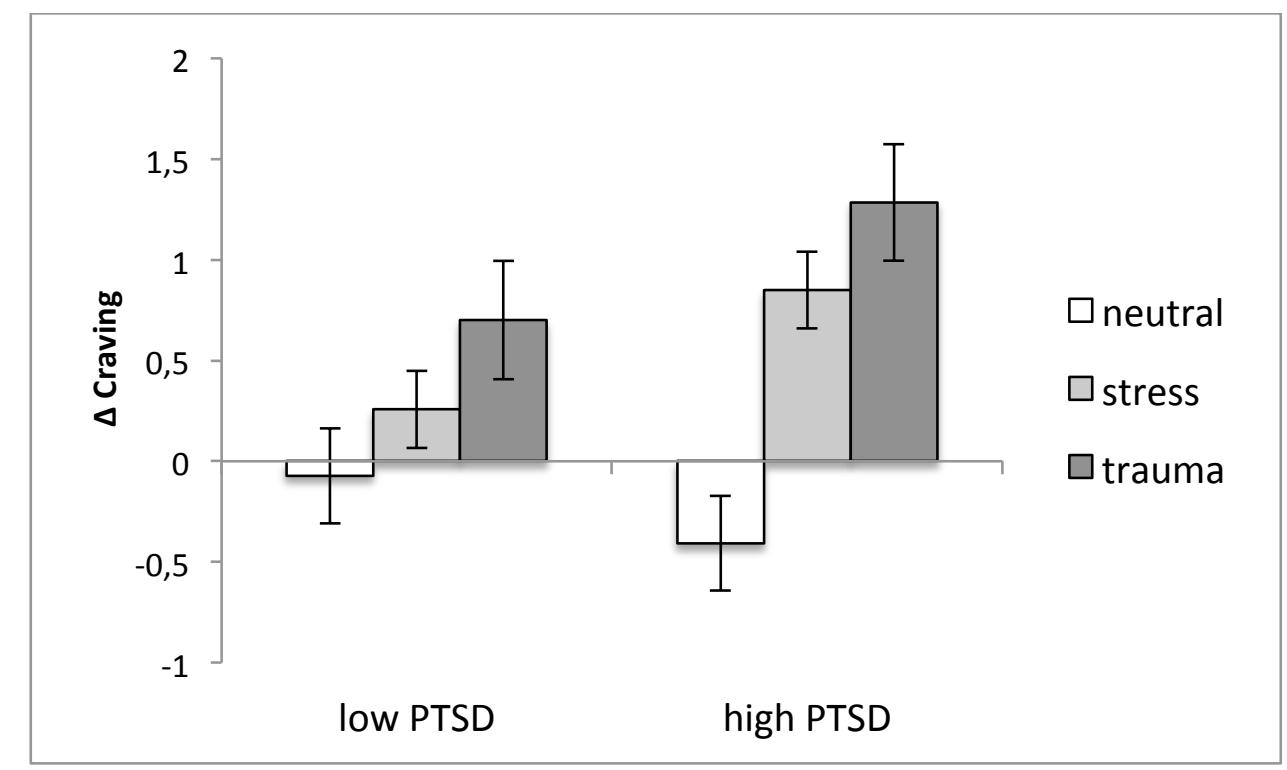

Figure 1. Effect of the conditions on craving (mean difference scores with SEM) for people high and low in PTSD.

\section{Correlations between tension and craving responses}

Table 5 displays the correlations between reported tension and reported craving for each cue (neutral, stress, and trauma). 
Table 5. Correlations between tension and craving responses.

\begin{tabular}{|c|c|c|c|c|c|c|}
\hline & $\begin{array}{l}\text { Craving } \\
\text { baseline } \\
\text { neutral }\end{array}$ & $\begin{array}{l}\text { Craving } \\
\text { after } \\
\text { neutral }\end{array}$ & $\begin{array}{l}\text { Craving } \\
\text { baseline } \\
\text { stress }\end{array}$ & $\begin{array}{l}\text { Craving } \\
\text { after stress }\end{array}$ & $\begin{array}{l}\text { Craving } \\
\text { baseline } \\
\text { trauma }\end{array}$ & $\begin{array}{l}\text { Craving } \\
\text { after } \\
\text { trauma }\end{array}$ \\
\hline $\begin{array}{l}\text { Tension } \\
\text { baseline } \\
\text { neutral }\end{array}$ & $\begin{array}{l}r=.41 \\
p=.000 \\
n=74\end{array}$ & $\begin{array}{l}r=.45 \\
p=.000 \\
n=74\end{array}$ & $n / a$ & $n / a$ & $n / a$ & $n / a$ \\
\hline $\begin{array}{l}\text { Tension } \\
\text { after } \\
\text { neutral }\end{array}$ & $\begin{array}{l}r=.45 \\
p=.000 \\
n=58\end{array}$ & $\begin{array}{l}r=.58 \\
p=.000 \\
n=58\end{array}$ & $n / a$ & $n / a$ & $n / a$ & $n / a$ \\
\hline $\begin{array}{l}\text { Tension } \\
\text { baseline } \\
\text { stress }\end{array}$ & $n / a$ & $n / a$ & $\begin{array}{l}r=.58 \\
p=.000 \\
n=74\end{array}$ & $\begin{array}{l}r=.56 \\
p=.000 \\
n=74\end{array}$ & $n / a$ & $n / a$ \\
\hline $\begin{array}{l}\text { Tension } \\
\text { after stress }\end{array}$ & $n / a$ & $n / a$ & $\begin{array}{l}r=.30 \\
p=.021 \\
n=59\end{array}$ & $\begin{array}{l}r=.39 \\
p=.002 \\
n=59\end{array}$ & $n / a$ & $n / a$ \\
\hline $\begin{array}{l}\text { Tension } \\
\text { baseline } \\
\text { trauma }\end{array}$ & $n / a$ & $n / a$ & $n / a$ & $n / a$ & $\begin{array}{l}r=.49 \\
p=.000 \\
n=74\end{array}$ & $\begin{array}{l}r=.43 \\
p=.000 \\
n=74\end{array}$ \\
\hline $\begin{array}{l}\text { Tension } \\
\text { after } \\
\text { trauma }\end{array}$ & $n / a$ & $n / a$ & $n / a$ & $n / a$ & $\begin{array}{l}r=.10 \\
p=.427 \\
n=59\end{array}$ & $\begin{array}{l}r=.27 \\
p=.038 \\
n=59\end{array}$ \\
\hline
\end{tabular}

\section{Discussion}

The aim of the current study was to gain more understanding about the link between trauma exposure, stress exposure and substance craving. Patients with SUD who experienced trauma in their life, were exposed to three different scripts (neutral, stress and trauma) on different assessment days. Craving and tension were measured before and after cue exposure. PTSD severity was included as a covariate in the analysis. We expected that high PTSD severity leads to more craving and tension after stress and trauma cue exposure, with highest craving after trauma cue exposure.

The results showed that patients scoring high on PTSD craved more throughout the experiment. So, even before any kind of induction patients with high PTSD severity craved significantly more than patients with less PTSD severity. As craving is an important predictor for relapse (Back et al., 2010; 
Schneekloth et al., 2012), this suggests that SUD patients with high PTSD severity belong to an especially vulnerable subgroup of patients. This main effect of PTSD severity on craving is not consistent with a prior study in which cued craving was measured in a sample with different levels of PTSD severity (Saladin et al., 2003). They only found an interaction effect between PTSD severity and trauma cued craving. This difference might be explained by the fact that we did our measurements on different assessment days while patients were unaware of the type of cue to which they would be exposed that day. This uncertainty might have induced feelings of craving. Also, Brady (2003) found no group differences (Alcohol + PTSD vs. Alcohol only) in craving after patients were subjected to a Cold Pressor Task, though this might be attributed to the use of a general stress test in Brady's study while we used personalized scripts.

Next, not only was there more craving in patients with high PTSD scores, our study showed that these patients also reported more tension in all conditions of the experiment. This is not very surprising since PTSD is characterized by the experience of negative mood and increased arousal (5th ed.; DSM-5 APA, 2013) and our results thus merely reflect the symptomatology of PTSD. It is possible that this increased level of tension precedes the craving because SUD patients with high PTSD severity have limited coping mechanisms available besides using substances to dampen their negative affect. Our correlation analysis partly confirms this hypothesis. This is in accordance with previous studies (Gielen et al., 2015a; Gielen, Krumeich, Tekelenburg, Nederkoorn, \& Havermans, 2015b; Ouimette, Finney \&, Moos, 1999; Staiger, Melville, Hides, Kambouropoulos \&, Lubman, 2009). Other cue reactivity studies in which tension was measured as a dependent variable found more specific cue reactivity results. Coffey $(2006,2010)$ and Nosen (2014) only found increased distress levels after trauma cue exposure but not after the neutral cue exposure. In Brady's study (2006) no group differences were found with regard to tension or distress. Again, the different experimental procedure might explain these different outcomes.

More interestingly, besides the main effects of PTSD severity, a significant three-way interaction was detected. Patients with high levels of PTSD craved more after exposure to both the trauma and the stress cue, compared to the neutral cue. This finding was specific for craving, no such interaction was found for tension. Not only exposure to traumatic triggers, but also more general stress cues appear especially risky for relapse in patients with high PTSD scores.

Finally, we found that patients scoring high on PTSD also craved significantly more than low PTSD scorers after being exposed to a personalized stress script. As to our knowledge, previous SUD/PTSD studies never included a personalized stress cue in the paradigm. The finding means that SUD/PTSD patients are more vulnerable for craving, and possibly relapse after stress induction than SUD patients 
with less PTSD severity. It is likely that patients who suffered from a past trauma and who learned that substance use can ease the pain never really learned to cope in a different way with negative feelings or cognitions. If this is indeed the case, this has important treatment implications. SUD/PTSD patients should receive extensive skill training, probably even before a past-focused therapy takes place. This is exactly what is suggested in a phase-oriented treatment for complex PTSD (Cloitre, Courtois, Charuvastra, Carapezza, Stolbach, \& Green, 2011).

We acknowledge that the cue elicited craving, though it was significant, was still limited. This is not a unique phenomenon; other researches who measured craving in the lab observed small increases in craving as well (Avants, Margolin, Kosten, \& Cooney, 1995; Coffey, Saladin, Libet, Drobes, Brady, \& Dansky, 1999; Coffey et al., 2006). It might be that stronger craving could be elicited with the addition of an in vivo drug cue or in the drug use context (Coffey et al., 2002; 2006; 2010; Nosen et al., 2012; 2014; Saladin et al., 2003). Nonetheless, it is important to notice that patients in our study were abstinent for some time (mean abstinence was around 3 months) and that the majority of our participants denied any craving in the last month. With these facts in mind, it seems remarkable and clinically relevant that relatively stable SUD patients with high PTSD scores still report an increase in craving after exposure to stress or trauma cues.

In sum, the results from the current study show that SUD patients with high PTSD severity have an overall higher tendency to crave and to feel tensed, even in neutral situations. Furthermore, we demonstrated that SUD/PTSD patients crave not only significantly more after being exposed to a traumatic trigger, which has been found before (Coffey et al., 2002; 2010; Nosen et al., 2012; Saladin et al., 2003), but also after more general stress cues, unrelated to the trauma. The increased craving after trauma cue exposure is a replication of previous research by Coffey $(2002,2010)$, Saladin (2003), and Nosen (2012) and our study adds that this effect endures even in a more heterogeneous sample and even without exposing patients to an in vivo drug cue. When translating the results to the life of SUD/PTSD patients, this finding means that a significant increase in craving can occur just by being triggered to a past trauma or general stress cues, even in the absence of drugs or alcohol. These results suggest how vulnerable SUD/PTSD patients are for relapse when PTSD is left untreated. Our findings thus suggest the importance of integrating PTSD treatment and SUD treatment. Moreover, it adds up to the literature suggesting that the training of new coping skills should be integrated in PTSD treatment. 


\section{Chapter 4}

\section{Perceptions of clinicians about the comorbidity between PTSD and Substance Use Disorder}

\section{Published as:}

Gielen, N., Krumeich, A., Havermans, R. C., Smeets, F., \& Jansen, A. (2014). Why clinicians do not implement integrated treatment for comorbid substance use disorder and posttraumatic stress disorder: a qualitative study. European Journal of Psychotraumatology, 5. 


\section{Nine Inch Nails - Hurt}

I hurt myself today

To see if I still feel

I focus on the pain

The only thing that's real

The needle tears a hole

The old familiar sting

Try to kill it all away

But I remember everything 


\begin{abstract}
Healthcare providers working in addiction facilities do not often implement integrated treatment of comorbid substance use disorder (SUD) and posttraumatic stress disorder (PTSD) while there is empirical evidence to do so. This study aims to get insight into the views of clinicians with regard to the diagnosis and treatment of PTSD in SUD patients. A qualitative research method was chosen. Fourteen treatment staff members of different wards of an addiction care facility were interviewed by an independent interviewer. Despite acknowledging adverse consequences of trauma exposure on SUD, severe underdiagnosis of PTSD was mentioned and treatment of PTSD during SUD treatment was not supported. Obstacles related to the underestimation of PTSD among SUD patients and to the perceptions of SUD clinicians concerning the treatment of comorbid SUD/PTSD were reported. It is concluded that SUD treatment centers should train their clinicians to enable them to provide for integrated treatment of SUD/PTSD.
\end{abstract}




\section{Introduction}

Post-Traumatic Stress Disorder (PTSD) is a frequent co-occurring disorder in patients who seek treatment for their Substance Use Disorder (SUD). Prevalence rates around 35\% are mentioned in previous studies (Bonin, Norton, Asmundson, Dicurzio \& Pidlubney, 2000; Driessen et al., 2008; Gielen, Havermans, Tekelenburg, \& Jansen, 2012, Ouimette, Coolhart, Funderburk, Wade \& Brown, 2007; Read, Brown \& Kahler, 2004; Reynolds, Mezey, Chapman, Wheeler, Drummond \& Baldacchino, 2005), while trauma exposure was found to be prevalent in $89-97.4 \%$ of SUD patients (Dansky, Saladin, Coffey \& Brady, 1997; Farley, Golding, Young, Mulligan \& Minkoff, 2004; Gielen et al., 2012; Read et al., 2004; Reynolds et al., 2005). SUD patients with PTSD often use substances to deal with the emotional pain caused by their trauma (Khantzian, 1997; Leeies, Pagura, Sareen, \& Bolton, 2010; Ouimette, Read, Wade, \& Tirone, 2010). Treatment prognosis is relatively poor in these patients (Najt, Fusar-Poli, \& Brambilla, 2010; Read et al., 2004). In comparison with SUD patients without PTSD, the comorbid diagnosis of SUD/PTSD is related with a higher percentage of overdoses, suicide attempts and more treatment days (Mills, Teesson, Ross, Darke, \& Shanahan, 2005).

Over the last decade a large body of research on the treatment of SUD patients with PTSD has accumulated (e.g., Henslee \& Coffey, 2010; Hien, Cohen, Miele, Litt, \& Capstick, 2004; Najavits, Gallop, \& Weiss, 2006; Mills et al., 2012). Integrated treatment focusing on both disorders simultaneously seems to provide a better outcome than treatment that focuses on one disorder at a time (Dass-Brailsford \& Myrick, 2010; Mills et al., 2012; Zatzick et al., 2004). In addition to the importance to treat SUD and PTSD simultaneously, previous research also documents the need for structural assessment of PTSD in every new SUD patient who enters treatment (Back, Waldrop, Brady \& Hien, 2006; Gielen et al., 2012; Ruzek, Polusny \& Abueg, 1998). Guidelines, focusing on the PTSD/ SUD comorbidity and aimed to improve the quality of healthcare, have been developed to inform clinicians about these research findings, in that way bridging the gap between theory and practice (Kivlahan \& Kaysen, 2012; Ruzek, Polusny, \& Abueg, 1998). These guidelines were also recently developed in the Netherlands (Snoek, Wits, Meulders \& van de Mheen, 2012).

Despite the bulk of evidence and the development of guidelines, relatively few substance dependence treatment centres have implemented an integrated treatment approach, leaving PTSD in most cases untreated during SUD treatment (Glover-Graf \& Janikowski, 2001; Najavits, Sullivan, Schmitz, Weiss \& Lee, 2004; Young, Rosen \& Finney, 2005). Furthermore, assessment for PTSD does not take place in every new patient and underdiagnosis is fairly common (Gielen et al., 2012; Young et al., 2005). Although it is not clear what the specific reasons are for this contradiction between theory and practice, 
we do know that, in general, translation of research into practice is difficult (Forsner, Hansson, Brommels, Wistedt \& Forsell, 2010; Rothrauff \& Eby, 2011).

One of the reasons why implementation of research findings is difficult to accomplish is that individual clinicians hold different opinions about how to manage their patients (Leentjens \& Burgers, 2008; Sorensen \& Kosten, 2011). This explanation is related to Arthur Kleinman's theory (Kleinman, 1980). According to Kleinman's explanatory model (EM) approach, every individual, professionals and patients alike, holds different beliefs about a particular illness. These beliefs, or "EMs" are shaped by an individual's beliefs and impact how this individual applies these beliefs in particular illness episodes. Kleinman (1980) identified 5 constructs that determine how an individual defines and approaches a (health) problem and that constitute an individual's EM. These constructs include: (1) notions about the aetiology of the illness, (2) ideas about symptom onset, (3) views about the pathophysiology of the illness, (4) perceptions about the course of illness and (5) the recommended treatment. Kleinman states that how an individual defines a certain illness influences how this illness will be dealt with (i.e. what kind of treatment one thinks appropriate and by whom and what kind of assessment criteria one applies to these actions, including notions of what can be expected of professionals in terms of practices, attitude and responsibilities). Kleinman focuses on the distinction between EMs of professionals and EMs of patients and how the discrepant beliefs influence the treatment of a certain disease. His model can also be used to study the perception of professionals of a particular health problem such as SUD/PTSD, their attitude regarding existing or new treatment protocols and procedures, the kind of criteria they use to assess effectiveness and quality of existing or new practices and procedures, their actual evaluation of procedures and protocols and the preparedness to adopt new treatment regimes.

This article focuses on clinicians' EMs of comorbid SUD and PTSD. To understand why substance abuse clinicians do not implement evidence based integrated treatment for patients with SUD/PTSD it is useful to get insight into their views. The goal of this study is to explore why healthcare providers working in the addiction field do not offer integrated treatment for SUD/PTSD. We also aim at being able to formulate implementation guidelines for addiction facilities. Since this is a field study of clinician's perceptions a qualitative research method is the most suitable. The EM approach will be used as a tool to identify points of improvement.

To our knowledge, this is the first qualitative study that explores the perceptions of SUD clinicians about how to treat patients with comorbid SUD/PTSD patients. Some researchers have previously used surveys with the same goal. Glover-Graf and Janikowski $(2001,2003)$ used the SACSCIH to survey substance abuse clinicians who work with victims of incest. Najavits, Norman, Kivlahan and Kosten 
(2010) and Young et al. (2005) used surveys (resp. the VA version of the Clinician Survey on PTSD and Substance Abuse and a survey developed by the authors) in VA (Veterans Affairs) settings to learn more about the screening, treatment and referral of SUD/PTSD patients. While these studies focused on different subjects (clinicians in VA settings and SUD clinicians working with victims of incest), in two other studies clinicians were surveyed about the treatment of SUD/PTSD. Najavits (2002) surveyed 147 clinicians using the Clinical Survey on PTSD and Substance Abuse and concluded that the treatment of SUD/PTSD was rated as more difficult to treat than either disorder alone. Interestingly, she also found that clinicians perceived more gratification than difficulty in working with this subgroup of patients. Back, Waldrop and Brady (2009) tested 423 clinicians using the same survey as Najavits (2002). They found that the issue on when and how to integrate the treatment of SUD and the treatment of PTSD was perceived as the most challenging.

In this exploratory study we made use of a topic list and specifically addressed the following themes: assessment of trauma, diagnosis of PTSD and treatment of PTSD. By use of semi-structured qualitative interviews, clinicians were asked about these topics in order to gauge the clinician's EM of SUD/PTSD. We questioned the current procedures, the responsibilities and the possible obstacles.

\section{Methods}

\section{Design and justification}

Since this study aimed to explore the individual perceptions of substance abuse therapists, we used a qualitative research method. Semi-structured in-depth interviews with open-ended questions were used. This research method best fitted the current exploratory research questions.

\section{Data collection and sampling}

The current research took place in the addiction care division of Mondriaan. Mondriaan is a large institution with different certified treatment centres in the whole region of southern Limburg in the southern part of the Netherlands (total population currently estimated at 607000). One of these centres is a large substance/behavioral dependence treatment facility. Staff members of different wards of this facility were included in this study. A purposeful sampling strategy was used to achieve a representative sample with work setting, position and years of experience as selection criteria. These criteria were chosen because of their assumed influence on a clinician's EM. A profile list of possible participants in terms of these selection criteria was made, and each ward was contacted to find participants with the desired profile. 
A total of 20 candidate participants received an email explaining the goals and procedure of the study. Two persons declined participation due to time restrictions and 3 were non-responders, leaving 15 staff members who were eventually interviewed. Owing to technical problems one interview could not be transcribed. Since a suitable saturation level was reached after 14 interviews non-responders were not replaced in this study. The characteristics of the sample are outlined in Table 1.

Table 1. Sample characteristics.

\begin{tabular}{|l|l|l|l|}
\hline & Work setting & Position & Experience \\
\hline 1 & MC /FPAC /DDW & Psychotherapist & $>5$ years \\
\hline 2 & CCW & Family system therapist & $>5$ years \\
\hline 3 & FPAC & Psychiatric nurse & $<5$ years \\
\hline 4 & IT & Social worker & $<5$ years \\
\hline 5 & DDW/ MC & Psychologist & $<5$ years \\
\hline 6 & CCW/ MC & Psychiatrist & $>5$ years \\
\hline 7 & AT & Psychologist & $>5$ years \\
\hline 8 & IT/ AT & Psychiatrist & $<5$ years \\
\hline 9 & CCW & Psychiatric nurse & $<5$ years \\
\hline 10 & IT, AT & Social worker & $>5$ years \\
\hline 11 & CCW & Psychologist & $>5$ years \\
\hline 12 & AT & Social worker & $>5$ years \\
\hline 13 & DDW & Psychologist & $<5$ years \\
\hline 14 & CCW/ MC & Unit manager & $>5$ years \\
\hline
\end{tabular}

Work setting: intake team (IT), clinical continuation ward (CCW), forensic psychiatric addiction care (FPAC), double diagnosis ward (DDW), motivational centre (MC) and ambulatory treatment (AT).

\section{Procedure}

From October 2008 until January 2009, the selected staff members were interviewed. To prevent bias, an independent trained interviewer questioned the participants (investigator triangulation). All participants provided informed consent and agreed that the interviews would be recorded on audiotape. After an ice-breaker opening question ("Can you tell me something about the procedure 
when a new patient enters treatment?") a topic-list was used to cover the formulated themes: assessment of trauma, diagnosis of PTSD and treatment of PTSD. We were interested in the current procedure, the responsibilities and possible obstacles. The interviewer, who was familiar with the organization, was instructed to ask open-ended questions and to approach the respondents with a natural curiosity and respect to ensure honest and frank answers. Further instructions included holding a natural fluency in the questions and to communicate clearly. When a participant gave answers outside the scope of the interview, the interviewer brought the conversation back to the subject. Examples of questions were: (1) At what moment in the treatment process do you refer patients for PTSD treatment? (2) What treatment do you judge to be ideal for patients with SUD and PTSD?, (3) What kind of tools do you use to diagnose PTSD? or (4) Who is, according to you, responsible to question patients about past trauma?

Frequent debriefing sessions were organized to optimize the quality of the interviews. To further improve the reliability of the results we used member checking: the interviewer was instructed to restate or summarize the answers of the respondent and then to question the respondent to determine accuracy. Each interview lasted between 15 and 30 minutes. An independent coworker made transcripts of each interview.

\section{Data analysis}

The transcripts were analysed using content analysis. We chose to categorize the data with inductive analysis. Pope, Ziebland and Mays (2000) describe this procedure in their article. We will now give a detailed description of how we analysed our data. The transcriptions of the interviews were read and first marginal notes were added. Initially, these were open codes. Whatever came up and suited the text segment was written in the margin. When this was done for all the interviews, sensitizing concepts were chosen, reflecting associations of marginal notes between the interviews. The following selective codes were created: (1) definition of comorbid SUD/PTSD and assumptions about the underlying cause, (2) suitable treatments, (3) responsibilities, (4) anamnestic phase, (5) diagnosis process and (6) preconditions.

For each interview, a new document was composed with the six concepts as headings, and the exact copies of the respective text fragments were copy-pasted below. Summaries of the text fragments were made, each fragment resulting in a one or two sentence summary. These summaries were then combined for all interviews, resulting in six documents with all summaries for each theme. The summaries were carefully checked for connections and a higher level of abstraction was reached with 
new categories. After that, a fluent text was written for each category with the short summaries serving as illustrations. When this was done for the six concepts, a presentation was prepared and by doing so, we came up with a new and clearer way to organize the results.

Concept 3 (responsibilities) and 6 (preconditions) could be merged in the other four concepts. Furthermore, it became clear that, besides the first concept (definition of illness), a subdivision in (a) 'current situation', (b) 'ideal/ desired situation' and (c) 'needs to achieve the desired goals' was suitable for the remaining 3 themes. Finally, during analysis, subdivision (b) and (c) were combined since these constructs were closely connected with each other. The final categories thus emerged as (1) definition and aetiology of comorbid SUD/PTSD, (2) anamnestic phase, (3) diagnosis process and (4) suitable treatments. These categories match with Kleinman's EM constructs, with the first category corresponding to Kleinman's aetiology/ course of illness/ symptom onset and pathophysiology EM constructs and categories 2-4 belonging to Kleinman's recommended treatment EM domain.

To account for a potential researcher effect, the transcribed interviews were independently analysed by two different investigators (investigator triangulation). Besides the analysis which is outlined in detail in this section, the fourth author analysed the information in another way: she sorted the interviews by treatment facility and made four subcategories: diagnostics, treatment, referral and other relevant notions. The main relevant topics for each facility were selected and final summaries were compared between treatment units to identify issues that pertained to the entire organisation. Although the two investigators used different analysing techniques, they did come up with comparable results and conclusions (Smeets, 2009).

\section{Results}

\section{Definition and aetiology of comorbid SUD/PTSD}

When we asked the interviewees to estimate the prevalence of trauma exposure and PTSD in their SUD caseload, the opinions differed quite a lot, with estimates of trauma exposure ranging between 0.5 and $100 \%$.

'Trauma, as in PTSD in the DSM, is rare. Maybe only $1 \%$ or even less.'

I assume that every patient who is treated here has ever been exposed to a traumatic experience.' 
Only one interviewee (a psychologist) mentioned an indirect estimate of PTSD in SUD patients and stated to have never met a SUD patient with PTSD. No other interviewees reported specific estimations about PTSD prevalence.

'I've never seen real PTSD. So, its prevalence is quite low.'

Holding the literature findings on prevalence rates of PTSD and trauma exposure in SUD patients in mind, we can conclude that the estimated prevalence rates (despite the wide range) in this study suggest a severe underestimation of the problem.

An important issue was how participants interpreted comorbid SUD/PTSD. Among the interviewees many views came up. One of these corroborated the self-medication theory. According to the clinicians that referred to this theory traumatized individuals use substances to numb negative feelings or to suppress intrusions. As a consequence, these patients never learn to handle their problems and their symptoms become chronic. In this interpretation PTSD symptoms are, in other words, understood as a mediating factor leading to craving and possible relapse.

Other interpretations were offered. One of these suggested that SUD patients often expose themselves to dangerous or trauma-prone environments (high risk hypothesis; Stewart \& Conrod, 2008). It was assumed that when this trauma exposure happens after initial substance use, substance use aggravates.

'And, indeed, you meet severely addicted people who often expose themselves to dangerous situations which increases the risk for trauma.'

Another interpretation referred to the neurobiological dimension of substance dependence. This interpretation referred to a pre-programmed biological vulnerability to develop a mental disorder (diathesis stress model; Roberts, Moore \& Beckham, 2007). In interpretations referring to the neurobiological model it was asserted that early trauma can disturb neurobiological systems (reward system, neurotransmitters, and sleep pattern), leading to different effects after substance intake and putting someone at risk for the development of a SUD (disturbed stress reaction hypothesis).

A final interpretation focused on an interaction between trauma, SUD and personality. Trauma exposure, it was asserted, results in a change in personality or even in the development of a personality disorder and this can make a person vulnerable for the development of an SUD. Personality in this definition can be understood as a mediating factor. 
These definitions have in common a presumed relationship between PTSD and SUD. No matter how they perceived the causality, participants agree that it is important to have knowledge about the trauma as it can increase understanding the patient's motivations contributing to his/her SUD. The clinicians also agree that PTSD symptoms make it particularly difficult for the SUD patient to get clean or sober.

The first main finding is that the majority of clinicians seem to have a reasonably good understanding about the interrelatedness between SUD and PTSD. Although the clinicians severely underestimate the prevalence of PTSD in their patients (Gielen et al., 2012; Reynolds et al., 2005), their ideas about the aetiology, symptom onset, pathophysiology and course of illness are in accordance with the literature (Langeland, 2009; Roberts, Moore \& Beckham, 2007; Stewart \& Conrod, 2008). The fact that clinicians refer to existing theoretical models, including the self-medication theory, the high-risk hypothesis, the diathesis stress model and the disturbed stress reaction hypothesis, adds to the conclusion that the interviewed clinicians are well aware of the negative influences of trauma exposure and PTSD symptoms on SUD. One would thus expect that clinicians will take PTSD into account during SUD treatment. But that is clearly not the case. Why not?

\section{Trauma anamnesis in SUD patients}

\section{Current situation}

According to the interviewees there is a more or less standard screening at intake of any patient. This screening interview typically includes questions about current symptomatology, family history, life history, et cetera. Although trauma exposure is not specifically enquired in this anamnesis, it does, however, often suggest itself to the attentive intaker. The clinicians notice that sometimes patients spontaneously report trauma exposure. When trauma is considered by the clinician, it is done very carefully and only superficially.

\section{'But to really ask deeper about the trauma is of course not done.'}

When a patient enters treatment, clinicians usually rely on intake reports (and/or on existing patient files) and do not ask about possible trauma. It is noted that patients who are known for years sometimes have an unknown case-history. Furthermore, the interviewed intake clinicians agreed that sometimes they decide not to enquire about trauma because of the delicacy of the matter. The trauma anamnesis is implicitly expected to be continued during later treatment. 
'When I notice that they (the patients) find it (the trauma anamnesis) very hard, then I find it quite a challenge to ask about trauma. They often don't see me anymore after the intake. So, I then decide not to go into it and leave it for the treatment phase.'

The opinions on the responsibility to ask about trauma history are mixed. The trauma anamnesis should be done by the intake clinician, by the psychologist concerned, by the case manager or by the individual mentor (in case of hospitalization). Another view is that anyone should do it.

With regard to the anamnesis of trauma exposure we can conclude that (1) trauma exposure is not directly questioned in new patients and intake clinicians do not use specific validated assessment tools, (2) clinicians seem to favour a very careful approach with regard to the trauma anamnesis, (3) in case of already known patients clinicians rely on former and possibly outdated patient files and finally, (4) the responsibility to enquire about trauma is indistinct. So it is clear that there is a lack of a protocol concerning the trauma anamnesis in SUD patients. It is possible that the previously mentioned underestimation of trauma exposure prevalence is related with this absence of trauma anamnesis protocol. Since most clinicians believe that PTSD is not a frequent problem in SUD treatment, they do not experience a need for a protocol to assess trauma. The cautious approach toward trauma, on the other hand, may reflect the supposed association between talking about trauma, increase of PTSD symptoms and consequently an increase in craving and possible relapse of addictive behavior. This thinking contrasts clinical guidelines (e.g., Gielen et al., 2012; Ruzek et al., 1998) and the scientific literature. McHugo and colleagues (2005) interviewed over 2700 SUD patients with co-occurring mental disorders about how trauma assessment was tolerated. The results of McHugo's study indicated that the assessment was not only well tolerated, but was even regarded as a positive experience by most patients.

Ideal-desired situation

When the interviewer specifically asked for the need to assess trauma during intake or treatment, the clinicians do agree that trauma anamnesis is essential and they add that every patient should be directly questioned about possible trauma. They also state that is very important to clearly report these facts in their patient files and to be aware that already known patients may have incomplete or out-of-date patient files. 
'But there are people who are for instance on methadone and have been in and out of treatment for over 10 years. Not a lot of attention is paid to their history and who knows what further lurks beneath the surface.'

Interviewees state that when trauma is not questioned during intake, the intake clinician should clearly communicate this with the responsible substance abuse clinician.

In contrast to current practice the desired ideal situation is in line with the guidelines and can lead to a better assessment of trauma exposure. Interviewees provide some suggestions on how to reach this goal. The responsibility to assess trauma should be defined more clearly and training on how to assess trauma is needed for the intake clinicians.

\section{Diagnosing PTSD in SUD patients}

\section{Current situation}

Clinicians emphasize that it is important to diagnose PTSD. The following procedure in the diagnosis process of a new patient is reported. First, the intake clinician, often a social worker or a psychiatric nurse, makes a temporary diagnosis based on an interview. In case of uncertainty, the patient is then referred to a psychiatrist. This psychiatrist only sees a minority of the patients and does not use any standardized assessment tools in reaching a diagnosis.

'They (the patients) rarely end up with me (the psychiatrist)...I have 12 hours for the treatment of 1000 patients. I expect there are a lot of patients among them that have a whole lot of (psychiatric) problems of which I'm not aware.'

When the temporary diagnosis does seem clear, the patient is discussed in a multidisciplinary team meeting, including a psychologist and a psychiatrist. During this discussion a final diagnosis is made. The psychiatrist holds the end responsibility to make a diagnosis.

It is acknowledged that, in case of previously known patients, the diagnoses of the old patient files are often used without further inquiry.

The interviewees indicate that during treatment the diagnosis is malleable. Team members sometimes signal trauma related symptoms in a patient and then refer the patient for further consultation to the psychiatrist or resident psychologist. This may result in a change in the patient's diagnosis. Again, both the resident psychiatrist and psychologist rarely use standardized assessment tools to reach a specific diagnosis. As for PTSD specifically, the interviewees mention that no specific PTSD questionnaires or interviews are available or known to them. This of course may result in 
misdiagnosis or as in the case of PTSD, severe underdiagnosis. Indeed, the interviewed clinicians are in agreement with regard to the severe underdiagnosis of PTSD in their patient group.

'I think our patient group is heavily under diagnosed.'

Finally, the interviewees state that diagnosis can be difficult in SUD patients because of the similarity between PTSD symptoms and addiction related symptoms (e.g., intoxication and withdrawal).

'It is difficult to disentangle PTSD and addiction symptoms. What is what?'

Ideal-desired situation

The necessity to screen for PTSD in every patient at different times (since a diagnosis can change over time) during treatment is stressed. Furthermore, clinicians are aware that they should be more alert for PTSD symptoms. Although the importance of diagnosing PTSD in SUD patients is recognized, three important difficulties can be indicated. Firstly, the clinicians describe only two evaluation moments in which PTSD can be evaluated and report that in already known patients their previous diagnosis is copied, not re-assessed. Different existing clinical guidelines suggest at least three separate evaluation moments in all patients and a continuous monitoring of symptoms (Schatzberg, Weiss, Brady \& Culpepper, 2008; Snoek et al., 2012).

A second problem is that clinicians at intake reach a diagnosis relying solely on an interview in which PTSD or trauma exposure is not explicitly questioned. Clinicians seem to rely heavily on their clinical judgment when diagnosing PTSD in SUD patients. The combination of the reported underestimation of PTSD prevalence and the inherent bias of clinical judgement (Dawes, Faust \& Meehl, 1989; Garb; 2005) make for a plausible explanation of the underdiagnosis of PTSD.

The third difficulty is that PTSD symptoms can be confused with intoxication and withdrawal symptoms. Although some SUD related symptoms mimic or overlap with PTSD symptoms (e.g. sleep disturbance, difficulty concentrating, feelings of detachment, irritability), PTSD is characterized by unique criteria: the exposure to a criterion A event and intrusive trauma related symptoms (4th ed., text rev.; DSM-IV-TR; American Psychiatric Association, 2000). To overcome this problem of symptom confusion, the assessment of PTSD is best done after a period of abstinence (Snoek et al., 2012).

PTSD treatment in SUD patients

Current situation 
The interviews with the clinicians make it clear that the treatment facility promotes sequential treatment in which SUD is treated before PTSD. The focus should be on SUD and patients are sometimes advised not to go into trauma therapy during SUD treatment.

'I usually gave the advice that trauma therapy was not an option on that moment.'

'Doing that, you may cause more misery than necessary. In cases like that I'm more inclined to cover the trauma with sand, a concrete layer, and to continue work upon that rotten foundation.'

Although the sequential view on treatment of comorbid SUD and PTSD is seen as outmoded by different interviewees, quite a few interviewees strongly argue against simultaneous treatment. To treat PTSD during SUD treatment is seen as too soon, harmful, counterproductive, unwise and distracting.

'Sometimes doing nothing is less harmful.'

'The real trauma therapy is not done here because it usually impedes addiction treatment.'

In contrast with this current practice and thinking, the guidelines are very clear: integrated treatment is the standard of care (Kivlahan \& Kaysen, 2012; Mueser, Noordsy, Drake \& Fox, 2003; Snoek et al., 2012).

Despite the fact that guidelines emphasize using pharmacotherapy only as an additional form of therapy (Snoek et al., 2012), the interviewed clinicians indicate that pharmacotherapeutic treatment is the current treatment of choice for PTSD in SUD patients. Interviewees state that neuroleptica and Selective serotonin Reuptake Inhibitors (SSRI's) are prescribed for PTSD in SUD patients, leading to a decrease of intrusions and other PTSD symptoms. Psychopharmaceuticals are also advised for traumarelated sleeping disorders.

Clinicians favour a present-focused approach for PTSD treatment above a trauma- or pastfocused treatment. This approach involves carefully exploring the impact of trauma, developing new coping techniques, focusing on the future, psycho-education, symptom reduction and increasing stability. Interviewees state the importance of not talking about the trauma with the patient and to restrict or refer patients when they do.

'In essence, you don't discuss the trauma.'

The interviewees note that in patients with more introspective skills the psycho-education can be further deepened to increase the understanding and to link trauma-related patterns with addiction. Therapy can then include social skills training or rational emotive therapy. 
According to the interviewees, the above-mentioned approach can be applied by all team members, under supervision of a psychologist or psychiatrist. There should, however, be a match between psychopathology and expertise.

Current research suggests that past-focused therapies, not present-focused therapies, should even in SUD patients - be the treatment of choice (Berenz \& Coffey, 2012; van Dam et al., 2012).

The arguments that are given against trauma therapy during SUD treatment suggest that clinicians favour a sequential, pharmacotherapeutical, or present-focused treatment approach for PTSD because they believe that a past-focused integrated approach might exacerbate PTSD symptoms promoting drug or alcohol craving and possible addictive behaviour relapse.

Ideal - desired situation

Although sequential treatment seems to be the current way of handling SUD/PTSD patients, clinicians do report that dividing the treatment of SUD and PTSD is artificial and difficult to bring into practice. Because of the high suffering of the patient and the close link between PTSD symptoms and relapse, both disorders should be treated simultaneously. The interviewees recognize the ethical responsibility to do a co-treatment of SUD and PTSD.

'...so we have the responsibility to treat everything.'

According to the interviewees, this simultaneous treatment should, ideally, be given intramurally in a double diagnosis ward where a long enough admission time is possible.

'But, of course, it's not always relevant. It depends on where, on which department, and with what goal, someone is hospitalized.'

If PTSD treatment takes place on an ambulatory basis, an emergency admission should be possible in case of severe psychological deterioration of the patient. Clinicians further express the need to have a treatment protocol for comorbid SUD/PTSD. In this protocol, special attention should be given to a no show procedure as it is expected that no shows may occur more often with this subgroup of patients. A clear and uniform view of the institution about how to deal with comorbid SUD/PTSD is another important condition that is highlighted. If the institution stimulates PTSD treatment during SUD treatment, more time and money should be reserved for this aim.

'Not much is done with that information (PTSD diagnosis) because we can't do so much with it, because we don't have the means.' 
Training and supervision is needed in order to increase the knowledge about PTSD and about the impact of PTSD treatment. Intervision is suggested to prevent clinicians from developing secondary PTSD.

'We can't actually do that much with trauma within our departments of addiction care. We do not have the time or the expertise.'

As in the previous sections, there is an explicit contrast between the current practice and the situation that is described as ideal. The suggestions that are provided offer good possibilities to alter the current practices towards a more evidence-based treatment. Lack of time, money and expertise are mentioned as reasons why integrated treatment is currently not offered to SUD/PTSD patients.

\section{Gap between theory and practice}

Almost every interviewee (12 out of 14 ) believes that trauma and SUD are interrelated. Most clinicians report that substance use is negatively influenced by PTSD symptoms or that substance use follows trauma exposure. According to Kleinman's model these statements should predict that treatment of PTSD takes place during SUD treatment. However, nearly all clinicians admit that PTSD treatment does not occur during SUD treatment.

Interviewee 1: $\quad$ 'It (trauma/PTSD) can be a maintaining factor (for addiction).'

'Sometimes doing nothing is less harmful.'

Interviewee 2: 'Trauma is always of influence.'

'I don't think you should work on trauma processing here. That's more something for after the treatment (of addiction).'

Interviewee 4: $\quad$ 'The real problems start when someone is going to detoxify...then the sorrow, the pain and the grief emerge.'

'We can't do that much with trauma...During treatment we thus only focus on substance use.'

\section{Discussion}

In order to understand why substance abuse clinicians do not implement evidence based integrated treatment for patients with SUD/PTSD, the current report explored how healthcare providers define the comorbid disorders and how their perceptions influence clinical practice. The purpose of this study was 
to unearth perceptions and practices regarding SUD/PTSD in order to improve implementation of best practice guidelines concerning the comorbidity of SUD and PTSD for addiction facilities.

This study suggests that two factors affect the underdiagnosis and under treatment of PTSD in SUD facilities, which is also reported in the literature (Gielen et al., 2012; Glover-Graf \& Janikowski, 2001; Najavits et al., 2004; Young et al., 2005). (1) Although most clinicians are well aware of the adverse consequences of trauma exposure and PTSD on SUD, in general, SUD clinicians are not aware of the high prevalence rates of trauma exposure and PTSD among their patients. As a consequence, PTSD is not a priority and adequate treatment protocols, specific diagnostic tools and even the clinical guidelines are not well known. (2) Clinicians believe that talking about past traumas elicits craving and possible relapse. This belief leads to a too careful or no approach of past trauma.

The fact that the interviewees advised specific improvements about the trauma anamnesis, PTSD diagnosis and PTSD treatment which resembled the SUD/PTSD guidelines, suggests that their theoretical knowledge might not be the most important reason for not following the clinical guidelines. The main hurdles appear to be practical: lack of time, money and expertise. It should be noted though that the interviews forced the clinicians to think about PTSD in SUD patients. This impromptu awareness of PTSD in their patients may have lead them to stress the importance of diagnosing and treating PTSD in SUD patients. Nonetheless, with regard to Kleinman's EM approach we can conclude that the views SUD clinicians hold about PTSD prevalence and the supposed negative influence of discussing traumata certainly affect how the clinician handles comorbid PTSD in SUD patients. The matter is however further complicated by the lack of means.

Unfortunately, we could not ask participants to determine the accuracy of the conclusions because too much time passed between the interviews and the analysis. Nonetheless, the present findings might provide important implications for SUD treatment facilities.

\section{Conclusions}

The results of this study confirm that PTSD treatment was not a focus during SUD treatment. Although clinicians were well aware of the adverse consequences of trauma exposure and PTSD on SUD, hindrances related to the underestimation of PTSD in SUD patients, a too careful approach and lack of time and money prevented an adequate diagnosis and treatment of PTSD. SUD facilities should therefore invest in the evidence based integrated approach of comorbid SUD/PTSD. The results of this study corroborate previous findings that indicate that SUD treatment facilities have a lot to gain investing in integrated/simultaneous SUD/PTSD treatment. Clinicians should be educated and trained to 
be able to assess PTSD/trauma, using reliable and valid measures, and to provide evidence-based SUD/PTSD treatment. Since successful implementation is also dependent on the perceptions SUD/PTSD patients hold, future research might focus on this topic. 


\section{Chapter 5}

\section{Coping skills, motives for substance use and symptom interplay: how patients with}

Substance Use Disorder and PTSD experience their reality

Gielen, N., Nederkoorn, C., \& Havermans, R. (submitted for publication). Coping, use motives and symptom interplay: Perceptions of patients with substance use disorder and post-traumatic stress disorder. 


\section{Morphine - Cure for Pain}

Where is the ritual

And tell me where where is the taste

Where is the sacrifice

And tell me where where is the faith

Someday there'll be a cure for pain

That's the day I throw my drugs away

When they find a cure for pain 


\begin{abstract}
Despite empirical support, integrated treatment of Substance Use Disorder (SUD) and Posttraumatic Stress Disorder (PTSD) is not sufficiently implemented in SUD facilities. To understand the reasons for this gap between theory and practice, in this study the perceptions of SUD/PTSD patients about the cooccurrence and the symptom interplay of the two disorders were explored. Seventy-two SUD patients with differing levels of PTSD severity filled out a survey and three self-report questionnaires pertaining to their perceptions. Regression analysis was used to test whether PTSD severity accounts for possible differences between perceptions about the link between SUD and consequences of trauma exposure. Patients perceive that substance use is effective in diminishing PTSD symptoms, they feel that abstinence does not improve PTSD symptomatology, and they hold positive expectancies regarding the effect of the substance on their PTSD symptoms. Considering these patients' perceptions, it is hardly surprising that SUD/PTSD patients are reluctant to start an integrated SUD/PTSD treatment. Treatment implications are discussed.
\end{abstract}




\section{Introduction}

Substance Use Disorder (SUD) and post-traumatic stress disorder (PTSD) are two severe and often longlasting psychiatric disorders that frequently co-occur (Driessen et al., 2008; Gielen, Havermans, Tekelenburg, \& Jansen, 2012; Read, Brown, \& Kahler, 2004). Patients with this particular comorbidity have a higher risk for relapse of addictive behavior when their PTSD is left untreated (Mills, Teesson, Ross, Darke, \& Shanahan, 2005; Read et al., 2004).

There is a strong rationale and empirical support for integrated SUD/PTSD treatment (van Dam, Vedel, Ehring, \& Emmelkamp, 2012; Torchalla, Nosen, Rostam, \& Allen, 2012). In spite of this, SUD clinicians still seem to favor a sequential treatment, treating SUD first and leaving the PTSD to other trauma treatment specialists (Gielen, Krumeich, Havermans, Smeets, \& Jansen, 2014; Young, Rosen \& Finney, 2005). There is a gap between clinical science and practice here. To bridge this gap, one needs to understand why research findings are not implemented in SUD facilities.

Three possible barriers hindering implementation of integrated SUD/PTSD treatment can be identified: (1) obstacles related to the clinician (e.g., lack of expertise), (2) obstacles related to the institution (e.g., institution gives priority to SUD treatment), and (3) obstacles related to the patients (e.g., shame, anxiety). Implementation of integrated SUD/PTSD treatment is likely to fail if not all three barriers are removed. Previously researchers surveyed and interviewed clinicians, thereby identifying different factors preventing implementation of integrated SUD/PTSD treatment. One particular factor appears to be the anxiety on the part of the clinician that SUD patients with comorbid PTSD are too unstable to handle both treatment for SUD and PTSD. Indeed, one seems afraid that discussing trauma with the patient may put him/her in jeopardy for a (re-)lapse (Back, Waldrop, \& Brady, 2009; Gielen et al., 2014; Najavits, 2002).

Although there are studies suggesting that discussing trauma will increase the patient's craving for alcohol or drugs (Coffey et al., 2002; Saladin et al., 2003), this does not compromise treatment outcome. Indeed, as mentioned above, there are several studies showing better overall treatment outcome with integrated SUD/PTSD treatment, compared to SUD treatment only (van Dam et al., 2012; Torchalla et al., 2012). Therapists appear wary not to harm their patients, as they should, but a good therapist should not be so cautious as not to provide treatment at all. But what do SUD patients with comorbid PTSD think on this issue? How do they view the role of PTSD in their SUD? And is it possible that the patients feed the concerns of the therapist by showing resistance in discussing their trauma?

Janikowski and Glover (1994) and Janikowski, Bordieri, and Glover (1997) studied the relationship between incest and substance abuse. They surveyed patients and concluded that patients 
perceive incest and SUD to be interrelated. Furthermore, most patients preferred individual treatment and were willing to talk about their incest past if their counselor would only ask about it. Opinions regarding integrated treatment were mixed. Patient barriers that could be identified were feelings of shame and concerns about confidentiality. In 1998, a survey was conducted by Brown, Stout, and Gannon-Rowley, investigating the relationship between comorbid SUD/PTSD and patient treatment preferences. The results of the study showed that patients perceive the two disorders to be functionally related and that patients prefer a simultaneous treatment of SUD and PTSD. Patients in this study too were concerned about feelings of shame, blame, and about the painfulness of talking about their past trauma. Together, this suggest that some patients might indeed give signals to the therapist that the trauma is not discussed easily, but also that if they feel secure, they are willing to start a trauma-focused treatment.

But even if patients are willing to talk about their trauma, do patients feel it is relevant to integrate SUD and PTSD treatment? How do patients perceive the interplay between trauma, PTSD and substance use?

Some important questions are relevant in the perspective. The self-medication theory, which implies that substances are used as a way to cope with the PTSD symptoms (Khantzian, 1997), does not seem to offer a full explanation of the link between PTSD and SUD (Berenz \& Coffey, 2012; DassBraisford \& Myrick, 2010; Jacobsen, Southwick, \& Kosten, 2001). Researchers seem to agree that a more complex relationship is more likely to explain the frequent comorbidity between SUD and PTSD (Berenz \& Coffey, 2012; Dass-Braisford \& Myrick, 2010; Torchalla et al., 2012). Furthermore, researchers have never asked SUD/PTSD patients to elaborate upon the issue of first substance use. What were their reasons to start using drugs or alcohol? Did trauma experience play a role in this? What is the link between PTSD symptoms and craving (and possibly relapse) according to SUD/PTSD patients? Finally, so far, it has not been tested whether SUD patients with and without PTSD differ on how they view the relation between past trauma and substance use. It would be interesting and relevant to know whether it is PTSD or mere trauma exposure that is responsible for a possible distinction in results.

The present study focuses on the ideas of SUD/PTSD patients by asking them: (1) what is the relation between your past trauma and the start of your addiction, and what are your motives to keep using the substance? And (2) how is your PTSD related to craving and relapse (defining relapse as first substance use after a period of abstinence, regardless of whether patients resume abstinence after this substance use)? In the current study, SUD patients with different levels of PTSD severity (all with trauma 
exposure) filled out a brief survey and three questionnaires pertaining to their motives for drug or alcohol use.

To reach a complete understanding of the perceptions of patients with the SUD-PTSD comorbidity, another study was conducted, focusing on the perceptions of SUD/PTSD patients as well but using a qualitative design. This study is published elsewhere (Gielen, Krumeich, Tekelenburg, Nederkoorn \& Havermans, 2015b).

\section{Methods}

\section{Data collection and sampling}

The patients who participated in this study, also took part in a larger experimental study (concerning trauma cue induced cravings; Gielen, Nederkoorn, Havermans \& Jansen, 2015c) in which 91 SUD patients with trauma exposure in their past were included. General information about participant enrolment is described in Gielen et al. (2015c). Since not all patients completed the full study, data of 72 SUD patients were used for the current study. Participants were all in clinical treatment for SUD. PTSD was diagnosed with the use of a validated self-report questionnaire (self-rating inventory for PTSD [SRIP]; Hovens, Van der Ploeg, Bramsen, Klaarenbeek, Schreuder \& Rivero, 1994) and a clinical interview (MINI-The International Neuropsychiatric Interview; Overbeek, Schruers \& Griez, 1999). With the combination of scores on both diagnostic tools three groups were formed: 38 patients scoring negative for PTSD on both the MINI and the SRIP (SUD/ no PTSD group), 23 patients scoring positive for PTSD on the MINI or the SRIP (SUD/ probable PTSD group), and 11 patients scoring positive for PTSD on both the MINI and the SRIP (SUD/PTSD group). After patients provided informed consent, they were asked to fill out a survey and self-report questionnaires. Patients received a voucher of 20 euro for participating in the experimental study and completing the questionnaires for this study.

Patients experienced different types of trauma in their past. They experienced at least two different types of traumatic exposures. The included patients were between 4 and 30 years old when they first encountered a traumatic experience and mean age was $13(S D=7.26)$. Their primary preferred psychotropic substance of misuse was first used at a mean age of 22 (SD $=8.31$ ), with ages ranging between 11 and 43 years. Table 1 shows the sample characteristics of this sample. 
Table 1. Sample characteristics.

\begin{tabular}{|c|c|c|c|c|c|}
\hline Demographi & SUD/PTSD & SUD/probable PTSD & SUD/no PTSD & Statistics & Sign. \\
\hline $\begin{array}{l}\text { Mean age } \\
\text { (SD) }\end{array}$ & $\begin{array}{l}37.50(11.17) \\
(n=11)\end{array}$ & $\begin{array}{l}39.08(8.23) \\
(n=21)\end{array}$ & $\begin{array}{l}41.14(8.97) \\
(n=37)\end{array}$ & $F(2)=.80$ & NS \\
\hline Gender (\%) & $(n=11)$ & $(n=23)$ & $(n=38)$ & & \\
\hline Male & 63.6 & 73.9 & 81.6 & NP & NP \\
\hline Female & 36.4 & 26.1 & 18.4 & NP & NP \\
\hline $\begin{array}{l}\text { Primary } \\
\text { substance ( } 9\end{array}$ & $(n=11)$ & $(n=22)$ & $(n=36)$ & & \\
\hline Alcohol & 72.7 & 59.1 & 41.7 & $X^{2}(2)=3.88$ & NS \\
\hline Heroin & 0 & 18.2 & 16.7 & NP & NP \\
\hline Cocaine & 0 & 13.6 & 22.2 & NP & NP \\
\hline Cannabis & 9.1 & 4.5 & 13.9 & NP & NP \\
\hline Speed & 9.1 & 0 & 2.8 & NP & NP \\
\hline Poly & 9.1 & 0 & 2.8 & NP & NP \\
\hline Benzo's & 0 & 4.5 & 0 & NP & NP \\
\hline
\end{tabular}

Note: Degrees of freedom vary depending on which questionnaires/items were filled out by the patients.

Not Possible: Chi-square could not be calculated since minimum 1 cell had expected count less than 5.

\section{Materials}

All patients filled out a survey and three self-report questionnaires to gain more understanding about the perceived link between SUD and PTSD. The self-report questionnaires focused on the motives for substance use, the strategies for handling negative emotions, and expectancies regarding the effect of substances upon PTSD symptoms.

\section{Survey}

A survey was composed with questions concerning the link between traumatic past and substance use. Specific questions were asked about onset of SUD, craving, relapse, and self-medicating to cope with trauma intrusions. For each of these questions multiple-choice answers were provided. The following main questions (translated form Dutch) were used in this study: 
- According to your experience, is there a connection between your traumatic past and the start of your addiction? $(0$, no , or 1 , yes)

- According to your experience, is there a connection between your traumatic intrusions and craving? (possible scores ranging from 1 , no connection at all to 5 , a very clear connection)

- According to your experience, is there a connection between your traumatic past and relapse? (possible scores ranging from 1 , no connection at all to 5 , a very clear connection)

Three final questions were asked about patients' experiences with the use of substances to dampen traumatic intrusions ( 0 , no, or 1 , yes), the effectiveness of this self-medication ( 0 , no, or 1 , yes), and about the relationship between PTSD symptoms and abstinence (more, less, same amount of symptoms during abstinence).

\section{Modified Drinking Motives Questionnaire - Revised (DMQ-R)}

We used an adjusted version of the Modified DMQ-R (Grant, Stewart, O'Connor, Blackwell, \& Conrod, 2007) to incorporate a complete range of motives patients might have to use substances. Five items were added from the Marijuana Motives Measure (Simons, Correia, Carey, \& Borsari, 1998) and 7 more items were added from the Amsterdam Motives for Drinking Scale-short (Ooteman, Koeter, Verheul, Schippers, \& van den Brink, 2006). Patients were asked to rate a list of 34 items with reasons for substance use on a 5-point scale (1, [almost] never - 5, [almost] always). Every item belonged to one of seven possible categories (enhancement motives, expansion motives, social motives, somatic motives, coping-anxiety motives, coping-depression motives, and coping-general motives).

\section{Cognitive Emotion Regulation Questionnaire - short (CERQ-short)}

To assess the cognitive emotion regulation strategies SUD/PTSD patients use, the CERQ-short (Garnefski \& Kraaij, 2005) was administered to the patients. The questionnaire consists of 36 items, each belonging to one of 9 scales (self-blame, other-blame, acceptance, refocus on planning, positive refocusing, rumination, positive reappraisal, putting into perspective, and catastrophizing). Patients are asked to rate each item on a 5-point scale (1, (almost) never - 5, (almost) always). To interpret scores we used the norm table representing psychiatric patients. The CERQ-short has proven to have a good reliability and validity (Garnefski \& Kraaij, 2006). 
The P-AEQ (Norman, Inaba, Smith, \& Brown, 2008) is a 27 item self-report questionnaire to assess the beliefs SUD/PTSD patients hold about the effects of substances with regard to their PTSD symptoms. The P-AEQ measures two dimensions: a positive factor, reflecting beliefs that substances have a positive effect on PTSD symptoms, and a negative factor, reflecting beliefs that substances have a negative effect on PTSD symptoms. Scores may range between 1 (totally disagree) and 5 (totally agree). We adjusted the P-AEQ to include other substances besides alcohol.

\section{Data management and analyses}

All analyses were conducted using SPSS 21.0 for Windows. We used backward regression analyses to test whether PTSD severity accounts for possible differences between perceptions about the link between SUD and consequences of trauma exposure. Other possible predictors were included in the regression model as well (current age, type of substance [depressant or stimulant], and gender).

Logistical regression tests were used to analyze categorical data (binomial or multinomial depending on the levels of the dependent variable). Several participants had one or more missing values for items of the different measures described above. Due to these missing values, sample sizes vary slightly between the analyses.

Tables of full models are revealed only when a significant effect of PTSD severity was found.

\section{Results}

\section{Beginning of addiction}

In the survey, SUD patients indicated whether their traumatic past relates to what they believe represents the origin of their addiction. Most SUD/PTSD patients responded that their trauma was a cause of their addiction. Likewise, the same belief that trauma caused substance abuse and dependence was reported by most SUD/probable PTSD and SUD/no PTSD patients. A logistical regression analysis was conducted to predict the perception that addiction was caused by a traumatic exposure using PTSD severity, current age, type of substance [depressant or stimulant], and gender as predictors. A test of the full model against a constant only model was not significant, indicating that the predictors could not distinguish between patients who felt that trauma caused their addiction and patients who felt that trauma and start of addiction were two independent factors $\left(X^{2}=3.99, p>.05\right)$.

All descriptives of this study are displayed in Table 2. 
Table 2. Descriptives.

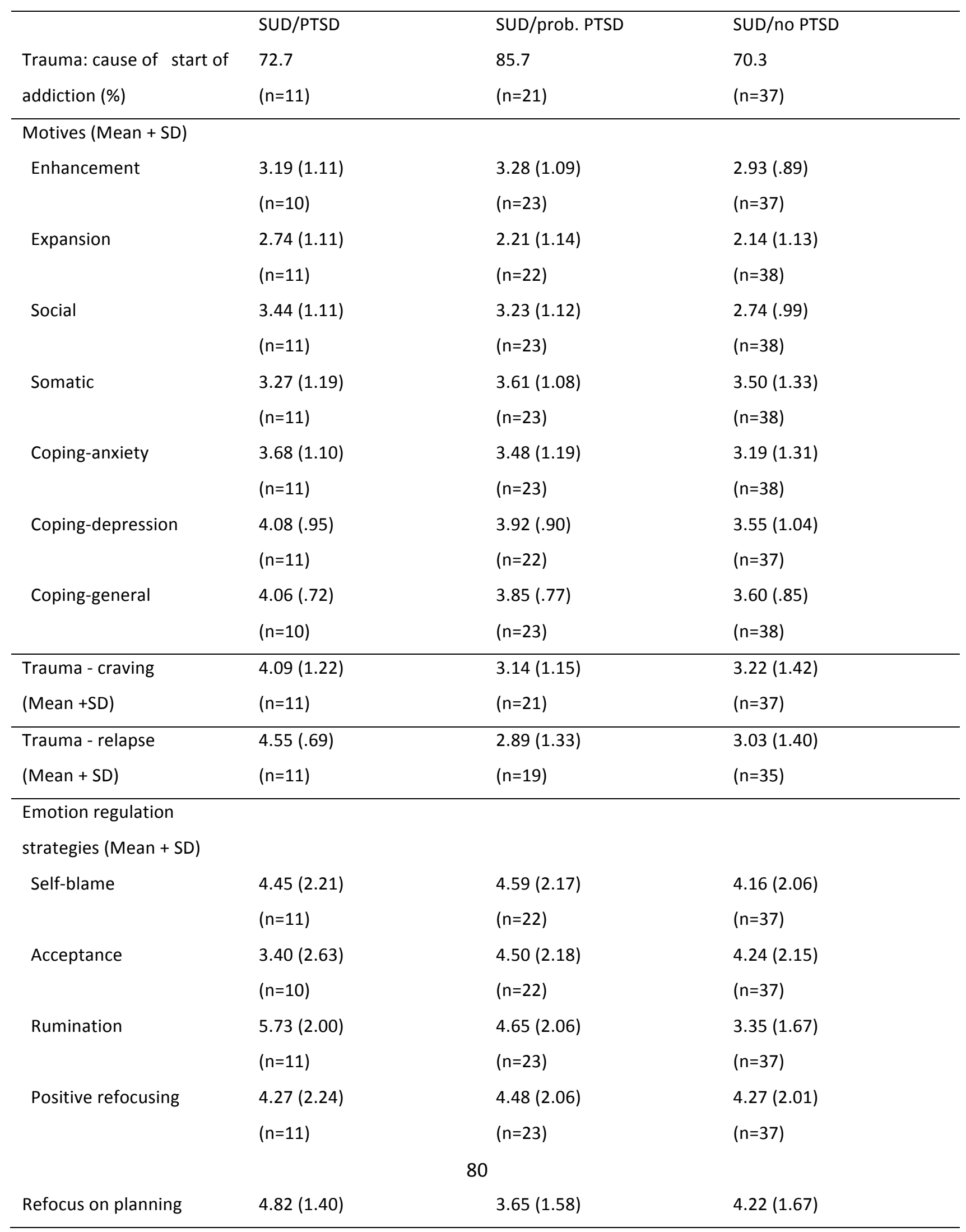




\begin{tabular}{llll}
\hline & $(n=11)$ & $(n=23)$ & $(n=37)$ \\
Positive reappraisal & $4.45(1.63)$ & $4.52(1.85)$ & $5.43(1.59)$ \\
& $(n=11)$ & $(n=23)$ & $(n=37)$ \\
Putting into perspective & $3.91(2.17)$ & $3.61(2.04)$ & $4.32(1.93)$ \\
& $(n=11)$ & $(n=23)$ & $(n=37)$ \\
Catastrophizing & $6.55(.69)$ & $5.09(1.90)$ & $4.68(1.78)$ \\
Other -blame & $(n=11)$ & $(n=23)$ & $(n=37)$ \\
& $5.27(2.24)$ & $4.43(1.80)$ & $4.00(1.56)$ \\
\hline Expectancies (Mean +SD) & $(n=11)$ & $(n=23)$ & $(n=37)$ \\
Positive expectancies & $3.38(.90)$ & & $3.26(.89)$ \\
Negative expectancies & $2.44(.83)$ & $3.64(.89)$ & $(n=32)$ \\
& $(n=10)$ & $(n=23)$ & $2.31(.83)$ \\
\hline
\end{tabular}

Note $n$ varies depending on which questionnaires/items were filled out by the patients.

Motives for continued substance use

Results from the DMQ-R indicated that coping motives (sum of coping-anxiety, coping-depression and coping-general) were mentioned most frequently as the primary motive for substance use, irrespective of having PTSD. However, as can be seen in Table 3, SUD patients use drugs or alcohol significantly more because of social motives when they score higher on PTSD severity. There were no significant differences between the PTSD groups with regard to other motives. Other predictors were, however, responsible for outcome differences in coping-anxiety motives (gender: $B=.73, S E B=.34, t=2.11, p=$ .039 , and type of main substance: $B=-.94$, SE $B=.37, t=-2.51, p=.014$ ) somatic motives (type of main substance: $B=-1.09, S E B=.36, t=-3.01, p=.004$ ) and enhancement motives (current age: $B=-.04, S E B$ $=.01, t=-2.92, p=.005)$. 
Table 3. Regression table with PTSD severity as significant predictor.

\begin{tabular}{lccc}
\hline & $B$ & $S E B$ & 6 \\
\hline Social motives - use & .40 & .17 & $.27^{*}$ \\
Relapse - trauma & .58 & .23 & $.31^{*}$ \\
Rumination & 1.19 & .31 & $.43^{* *}$ \\
Positive reappraisal & -.63 & .27 & $-.27^{*}$ \\
Catastrophizing & .90 & .28 & $.36^{* *}$ \\
Other-blame & .58 & .29 & $.24^{*}$ \\
\hline
\end{tabular}

$* p<.05 \quad * * p<.005$

Opinions regarding the link between craving and relapse and trauma

We asked the included patients the following two questions: (1) According to your experience, what is the connection between traumatic intrusions and craving? (2) According to you, what is the connection between a traumatic past and relapse? No significant differences between the groups were detected with regard to craving ( $B=.27, S E B=.22, t=1.20, p=.234)$. On average, SUD/PTSD patients felt that relapse followed traumatic memories very often. This differed significantly from SUD/ probable PTSD, and SUD/no PTSD patients. As shown in Table 3, PTSD severity was responsible for predicting this difference.

Most SUD/PTSD patients (90.9\%) stated that craving or a relapse happened always or often as a consequence of their traumatic past as compared to $63.6 \%$ of SUD/probable PTSD patients and $57.9 \%$ of SUD/no PTSD patients. A logistical regression analysis showed however that these differences were not significant, indicating that the predictors could not distinguish between patients who perceived that craving or relapse happened as a consequence of trauma and patients who perceived the two factors as independent $\left(X^{2}=5.29, p=.381\right)$.

\section{Perceived relationship between SUD and PTSD}

We asked the patients whether they ever used a substance after experiencing traumatic intrusions. We found that $90.9 \%$ of the SUD/PTSD patients reported having used drugs or alcohol after an intrusion (vs. $87.5 \%$ in the SUD/probable PTSD group and $75 \%$ in the SUD/no PTSD group). Logistical regression analysis showed that these results did not depend on PTSD severity or any of the other predictors (i.e., current age, type of substance [depressant or stimulant], and gender) $\left(X^{2}=3.45, p=.063\right)$.

$90 \%$ of the SUD/PTSD patients with this experience added that their substance use was, at that moment, effective in diminishing the intrusion. This percentage was lower in the other groups $(76.5 \%$ in 
the SUD/probable group and $77.3 \%$ in the SUD/no PTSD group), but this outcome was not predicted by PTSD severity or any of the other predictor variables $\left(X^{2}=-1.37, p=.242\right)$.

We further asked the patients to state whether they experience more, less, or the same amount of PTSD symptoms during substance use and abstinence. We found that in the SUD/PTSD group, $36.4 \%$ reported fewer symptoms during substance use, $45.5 \%$ experienced more PTSD symptoms, and in $18.2 \%$ of the SUD/PTSD patients, the symptoms did not change during substance use. Multinomial logistical regression analysis showed that this outcome did not depend on one of the predictor variables (i.e., PTSD severity, current age, type of substance [depressant or stimulant], and gender; with all $X^{2} s \leq 3.65$, all $p$ 's $>.05$.

\section{Cognitive emotion regulating strategies}

Data analysis of the answers on the CERQ-short indicated that SUD/PTSD patients scored significantly higher on rumination, other-blame and catastrophizing than SUD/no PTSD patients. Furthermore, SUD/PTSD patients scored significantly lower on positive reappraisal. As can be seen in Table 3 these differences were all predicted by PTSD severity. "Positive reappraisal" was also predicted by current age $(B=-.05, S E B=.02, t=-2.49, p=.015)$. Differences on "Positive Refocusing" were predicted by type of main substance $(B=-1.48, S E B=.63, t=-.28, p=.023)$.

\section{Expectancies of effect of substances on PTSD symptoms}

Data on the P-AEQ indicated that SUD patients with trauma exposure in their past have more positive than negative expectancies about the effect of their favorite substance on trauma related symptoms. None of the predictor variables predicted this relationship with all $B^{\prime} s \leq .46$, all $S E B^{\prime} s \leq .28$, all $t^{\prime} s \leq 1.18$, and all $p^{\prime} s>.05$.

\section{Discussion}

This exploratory study aimed to get more insight into the perceptions of SUD/PTSD patients concerning their SUD and PTSD symptoms. SUD patients with different levels of PTSD severity were asked to fill out a survey and self-report questionnaires. Two research questions were formulated. The first research question in the present study concerned the perceived relation between SUD and past trauma. Although the majority of SUD/PTSD patients claimed that their traumatic past is a cause of their SUD, this finding was not specific. SUD patients with lower levels of PTSD severity reported likewise that their traumatic past was a cause of their excessive substance use. SUD patients with a traumatic past thus consider 
trauma to be a causal factor in their addiction, regardless if the trauma led to PTSD. Almost all SUD/PTSD patients in the study reported using substances to cope with the negative consequences of their traumatic past. This self-medicating behavior is deemed effective by a large majority of the patients. The same holds, however, for SUD patients with lower levels of PTSD severity.

Comparing the results between groups we found that SUD/PTSD patients used substances significantly more because of social motives. This finding can be explained by the detachment, distrust, hyperarousal and anxiety PTSD patients often report. It seems that SUD/PTSD patients use substances to feel more at ease in social environments.

Furthermore, SUD/PTSD patients have less adaptive cognitive emotion regulation strategies, hold positive expectancies regarding the effect of the substance on their PTSD symptoms, and abstinence does not improve PTSD symptomatology. It is therefore hardly surprising that SUD/PTSD patients find it hard to stop using substances.

Our second research question concerned craving and relapse as a consequence of a traumatic past. A clear link exists between craving, relapse, and PTSD symptoms but also SUD patients without (or with less severe symptoms of) PTSD experience craving when being reminded of their traumatic past. The link between relapse and PTSD related symptoms on the other hand, is specific for patients with the comorbidity.

Important treatment implications can be drawn from this study. Since SUD patients experience a clear link between their SUD and their traumatic past, it seems fit to introduce a trauma-focused component in their SUD treatment, even for patients with lower levels of PTSD severity. Psychoeducation about the link between SUD and past trauma can be a first and non-invasive step towards a better understanding of symptom interplay. Furthermore, although almost all SUD patients with a traumatic past reported the effective use of substances to cope with intrusions, the inadequacy of coping skills was very specific for SUD patients with high PTSD severity, implying that coping skills training should be an essential part of SUD treatment, especially for SUD/PTSD patients. From this we can deduce that a phase-oriented PTSD treatment seems most appropriate for SUD/PTSD patients. The first phase of this treatment consists of improving coping skills and ameliorating emotion regulation strategies (see Cloitre, et al., 2011).

To summarize, early trauma may engender individuals to develop an SUD (Brady, Back, \& Coffey, 2004). In line with self-medication theory, patients with comorbid SUD/PTSD report that substance use is a maladaptive though effective way to cope with negative emotions, intrusive memories, and other PTSD symptoms in that it decreases or dampens these symptoms. This of course reinforces substance 
use and raises the risk of developing dependence. When PTSD is left untreated, PTSD symptoms pose a heightened risk for relapse of addictive behavior. Like other SUD patients (see Sinha, 2000), SUD/PTSD patients do not seem to have learned alternative ways of coping with negative emotions and cognitions. The efficacy of addiction treatment programs would likely benefit from including some form of PTSD treatment for SUD patients with clear (and sometimes debilitating) trauma exposure. The finding that SUD/PTSD patients perceive a strong interrelatedness between trauma exposure, SUD and PTSD symptoms, suggests that they would identify this additional PTSD therapy as useful and relevant. Based on this study, we can thus conclude that possible barriers for the implementation of an integrated SUD/PTSD treatment are probably not to be found in patients. 


\section{Chapter 6}

\section{Patients speaking: how they perceive the relationship between trauma, substance abuse, craving, and relapse}

\section{Published as:}

Gielen, N., Krumeich, A., Tekelenburg, M., Nederkoorn, C., \& Havermans, R. (in press). How patients perceive the relationship between trauma, substance abuse, craving, and relapse; a qualitative study. Journal of Substance Use. 


\section{Pink Floyd - Comfortably Numb}

There is no pain you are receding.

A distant ship, smoke on the horizon.

You are only coming through in waves.

Your lips move but I can't hear what you're saying.

When I was a child I caught a fleeting glimpse

Out of the corner of my eye.

I turned to look but it was gone

I cannot put my finger on it now

The child is grown, the dream is gone.

I have become comfortably numb. 


\begin{abstract}
In this qualitative study, patients with comorbid substance use disorder (SUD) and post-traumatic stress disorder (PTSD) were interviewed on their ideas about the link between SUD and PTSD. Although they clearly reported self-medication, they also gave a more complex description of how they believe their PTSD influences their SUD. The results suggest that SUD/PTSD patients believe they did not start using substances because of their experienced traumas or PTSD, but that PTSD symptoms are nonetheless important in the maintenance of their addictions. A clear link exists between craving, relapse, and PTSD symptoms. SUD/PTSD patients would prefer a "whole-person approach" when being treated for their PTSD. It is suggested that the integration of skills training and attention for patients who are fearful of PTSD treatment might improve SUD/PTSD treatment results.
\end{abstract}




\section{Introduction}

LaCoursiere, Godfrey, and Ruby (1980) were probably the first authors to describe the link between substance use disorder (SUD) and post-traumatic stress disorder (PTSD). Starting in the early 1980s, many authors published articles that described the heightened prevalence of PTSD and trauma exposure among SUD patients and emphasized the vulnerability of this patient subgroup (Bonin, Norton, Asmundson, Dicurzio, \& Pidlubney, 2000; Gielen, Havermans, Tekelenburg, \& Jansen, 2012; Ouimette, Brown, \& Najavits, 1998; Taber, McCormick, \& Ramirez, 1987).

Recent years have seen the publication of review articles that once again emphasize the need to treat SUD and PTSD in a simultaneous and integrated manner (Dass-Brailsford \& Myrick, 2010; Torchalla, Nosen, Rostam, \& Allen, 2012; van Dam, Vedel, Ehring, \& Emmelkamp, 2012). Despite that recommendation, addiction treatment facilities still do not seem to offer integrated treatment for SUD/PTSD patients (Gielen, Krumeich, Havermans, Smeets, \& Jansen, 2014; Young, Rosen, \& Finney, 2005). Why does this gap between science and clinical practice exist? And, even more importantly, what should be done to bridge this gap?

It is relevant to learn more about how SUD/PTSD patients perceive the link between their traumatic pasts and their SUD. How do patients feel about receiving integrated SUD/PTSD treatment? It is clear from previous studies that we can learn a lot about the relationship between trauma exposure and addiction by listening to patients' narratives (Larrabee \& Bolden, 2001; Sofaer \& Firminger, 2005).

Some researchers have queried the views of SUD patients with a traumatic past. For example, the perceptions of patients who suffered sexual abuse as children and now have SUD have been the focus of a qualitative study by Jarvis, Copeland, and Walton (1998) and a case study by Teusch (2001). Both research teams noted the important role of self-medication and coping for SUD patients with comorbid PTSD, and both emphasized the complexity of this subgroup of patients. They noted that feelings of impaired self-esteem, hopelessness, shame, and guilt were possible barriers to PTSD treatment.

Hall (2000) and Harris, Fallot, and Berley (2005) conducted qualitative studies that only included traumatized women with SUD. Hall interviewed 20 women who had been abused as children and concluded that the abuse was relevant to their current life difficulties. Negative ideas about self and the future were manifested as possible barriers for treatment. The study by Harris et al. focused on recovery and relapse prevention. They stressed the importance of interpersonal connectedness to sustaining an abstinent lifestyle. This study found that depression and a lack of personal control were possible barriers to successful treatment of these patients. 
We recently conducted a survey study in which we asked SUD patients with traumatic pasts about how they perceive the interrelatedness between their traumatic pasts and SUD (Gielen, Nederkoorn, \& Havermans, 2015b). This study included 72 SUD patients with different levels of PTSD severity. We found that the SUD patients with severe PTSD used substances to cope with general stress and had less adequate coping skills than SUD patients with less severe PTSD. Furthermore, this study found that SUD/PTSD patients perceive a clear link between their past traumas and previous relapse experiences.

Some questions still remain. Not much is known about what patients actually expect or prefer during their treatment (Dass-Brailsford \& Myrick, 2010). Furthermore, patient surveys and interviews have shown that while some patients favor integrated treatment, other patients mainly mention obstacles and barriers related to PTSD treatment during their SUD treatment (Brown, Stout, \& GannonRowley, 1998; Hall, 2000; Harris et al., 2005; Janikowski, Bordieri, \& Glover, 1997; Janikowski \& Glover, 1994; Jarvis et al., 1998; Teusch, 2001). It is not clear what determines this distinction. Furthermore, although previous studies have found that SUD/PTSD patients perceive a link between their past traumas and their SUD (Brown et al., 1998; Gielen et al., 2015b; Janikowski et al., 1997; Janikowski \& Glover, 1994), we have no in-depth understanding of how SUD/PTSD patients evaluate the role of PTSD in the development and continuation of their substance dependency. Do patients think PTSD was involved in the onset of their drug use, the transition into an addiction or the continuation of the addiction and relapses? These missing factors could be important to the successful implementation of integrated SUD/PTSD treatment.

We determined that a qualitative research design was most suitable to reach this in-depth understanding of the perceptions of SUD/PTSD patients. We then formulated two research questions: (1) According to SUD/PTSD patients, why did they start using drugs/alcohol and why did they keep using the substance? and (2) What types of treatment would SUD/PTSD patients prefer and why? To help answer these questions, an independent researcher conducted semi-structured interviews with SUD/PTSD patients.

\section{Methods}

Data collection and sampling

We recruited 432 SUD patients for a prevalence study. These patients were all in treatment for SUD and were asked to fill out a PTSD questionnaire; $36.6 \%$ of them had a positive diagnosis for PTSD (Gielen et al., 2012). From this group, we selected 10 patients using the following selection criteria: main product 
(i.e., previously preferred psychotropic substance for misuse), age, and treatment facility. This purposeful sampling strategy was used to achieve a heterogeneous sample of treatment-seeking SUD/PTSD patients with differing characteristics. One patient was excluded from further analysis because that patient showed psychotic symptoms during the interview. All interviewed patients had experienced type II trauma with multiple, prolonged, or chronic traumas happening throughout their lives; these included emotional neglect/abuse, sexual abuse, physical abuse, imprisonment, and/or being confronted by or witnessing death. Most patients had experienced a traumatic event in their childhoods and started using drugs and/or alcohol as teenagers.

The SUD/PTSD patients we interviewed experienced many current complaints and problems. Most of them stated that their problems had already started in childhood. They went on to experience difficulties in their relationships and their families. Some had legal problems and others reported problems related to their social environments and their finances. Finally, the patients were troubled by health-related issues. Table 1 shows the sample characteristics of the interviewed individuals.

Table 1. Sample characteristics.

\begin{tabular}{l|llll}
\hline & Gender & $\begin{array}{l}\text { Preferred } \\
\text { substance }\end{array}$ & Age & Treatment facility \\
\hline 1 & Male & Poly & 36 & ambulatory treatment \\
2 & Female & Poly & 49 & double diagnosis ward \\
3 & Male & Alcohol & 64 & outpatient treatment \\
4 & Male & Poly & 29 & reintegration training \\
6 & Male & Poly & 37 & forensic psychiatric addiction care \\
7 & Male & Poly & 53 & double diagnosis ward \\
8 & Male & Poly & 28 & ambulatory treatment \\
9 & Male & Poly & 46 & motivational center
\end{tabular}

Procedure

Patients were interviewed by an independent trained interviewer, a student working on a Master's degree in mental health who had frequent consultations with her supervisors. Interviews were 
audiotaped for later transcription. A topics list was used so that the following themes were included in the interviews: the perceived link between SUD and PTSD, the perceived reasons for first substance use and continued substance use, the perceived link between trauma and craving and relapse, and preferred treatment. The interviewer, who was familiar with the organization, was instructed to ask open-ended questions and to approach the participants with a natural curiosity and respect to ensure honest and frank answers.

Each semi-structured in-depth interview lasted approximately 45 minutes. When a participant gave answers outside the scope of the interview, the interviewer brought the conversation back to the subject. At the end of the interview, patients were debriefed and given information about PTSD and treatment options. An independent co-worker transcribed each interview.

Patients gave informed consent for study participation. They received gift vouchers worth $€ 20$ in exchange for their participation.

\section{Data analysis}

We analyzed the data in a similar way as in our previously published study (Gielen et al., 2014). We used content and inductive analysis to analyze the qualitative data (Pope, Ziebland, \& Mays, 2000). We read the interview transcripts and added marginal notes (primarily open codes). Whatever came up and fitted the text segment was written in the side-line. When, for example, the interviewee talked about the fact that he preferred a female therapist, the words "gender clinician" were written in the margin.

After we completed this analysis for all the interviews, we chose sensitizing concepts that reflected associations in the marginal notes between the interviews. The sensitizing concepts were: (1) reasons for continued substance use, (2) connection trauma - substance use, (3) craving, (4) relapse, (5) trauma treatment, (6) consequences of trauma, (7) ages of trauma and first substance use, (8) reasons for first substance use, (9) course of substance use, (10) type of trauma, (11) PTSD symptoms, and (12) symptom decrease after substance use.

For each interview, we created a new document with the concepts as headings and pasted exact copies of the respective text fragments below. We summarized the text fragments; each fragment resulted in a one- or two-sentence summary. In this phase, we combined concepts 2 and 12, as well as concepts 6 and 11 . These summaries were then combined for all interviews, resulting in 10 documents with all the summaries for each theme. The summaries were carefully checked for connections and a higher level of abstraction was reached, with six new subcategories emerging. These final categories 
were: (1) first substance use, (2) course of SUD, (3) motives for continued substance use, (4) craving and relapse, (5) link between SUD and PTSD, and (6) treatment preferences.

To account for a potential researcher effect, the transcribed interviews were independently analyzed by two different investigators (i.e., investigator triangulation). The first author analyzed the data as outlined in this section, while the third author analyzed the data using Kleinman's (1980) clinical core functions as the guiding principle. Although the two investigators used different analysis techniques, they came up with comparable results and conclusions.

\section{Results}

\section{First substance use}

Analysis of the interviews showed that SUD/PTSD patients often started using alcohol/drugs as teenagers. They described no stress- or trauma-related triggers; first substance use occurred without any provocation and was seen as part of experimenting in one's youth. Some stressed that their first substance use happened in a social environment. For instance: "I started using hashish around age 15 or 16. ... I just did it, I didn't really think about it."

\section{Course of SUD}

SUD/PTSD patients described how their substance use gradually increased. They often started using cannabis or alcohol, and then tried other drugs. This phenomenon relates to the gateway theory in which less deleterious drugs pave the way for the use of more dangerous drugs (Vanyukov et al., 2012). It is important to note that only one respondent identified trauma as a deteriorating factor. The other patients described diverse factors that caused an increase in their substance use (e.g., receiving the monthly salary, working as a prostitute, burn-out, change in the family situation, house parties, withdrawal, friends who used substances). Factors related to improvement and abstinence were pregnancy, military service, and health problems.

"It became more when I was 18 because of the house parties. We drank, sniffed, smoked, and used XTC really hard. When I joined the army it was over. ... After military service, I met a friend who used heroin and I started using it too."

\section{Motives for continued substance use}

Patients were asked to elaborate upon their reasons or motives for substance use. Their motives can be divided into social motives (e.g., social pressure, belonging, parties, coziness, less social anxiety), 
addiction-specific motives (e.g., withdrawal, loss of control, substance cues, obsessive behavior, taste and smell), expansion motives (e.g., expanding one's consciousness), enhancement motives (e.g., kick, reward, the feeling, opportunism, euphoria, boredom alleviation), and coping motives. Motives in this last category were most frequently reported. Respondents used drugs or alcohol to forget, to escape reality, to survive, to cope with difficulties, to feel safe, to have more self-confidence, to dampen negative emotions, or to be able to sleep, rest, or relax. Some of the respondents mentioned these coping motives in specific relation to trauma, while others seemed to generalize it to all negative emotions and thoughts.

"I drank to stop my negative feelings and to not be burdened by them. I don't allow myself to feel anything and I use alcohol as a means to get rid of my feelings."

Opinions regarding the link between craving and relapse and trauma

Patients were asked about their experiences with craving and relapse. They stated that cravings could happen after drug or alcohol cue exposure or during withdrawal. Some SUD/PTSD patients specifically mentioned that cravings occurred when they were confronted with trauma cues (e.g., intrusions): "Memories and nightmares precede my cravings."

Relapse was described as a conscious choice, something that was usual and that could happen at any moment after a period of abstinence. They described specific reasons for relapse, which matched the motives for continued substance use and had a clear focus on coping motives. Other reasons for relapse were the end of a pregnancy and revenge after relationship problems. Patients stated that the chance of another relapse became higher every time they broke their abstinence rule. One patient clearly described his negative thoughts about himself after a relapse: "I'm weak; I'll never get rid of my addiction." Another patient stated:

“I continually relapsed because I couldn't forget these people, couldn't forget my problems. I got stuck in using drugs."

\section{Perceived relationship between SUD and PTSD}

The interviewed patients clearly described examples of how they self-medicated themselves with drugs or alcohol to cope with the negative consequences of their traumatic pasts. Patients were aware that the positive effects they experienced from drugs and alcohol are superficial and temporary. 
"When I use, I think about nothing, not about the negative things, the things that happened, the memories... At that moment, I feel great! But it only lasts for a short time and I feel depressed when it's over."

Some patients stated that PTSD symptoms do not diminish during intoxication. It is important to note that some patients only became aware of the link between their traumatic past and their substance use later in their lives. Their substance use typically increased after such a revelation.

\section{Treatment preferences}

None of the interviewed patients had been treated for their PTSD. One patient even mentioned that this was the first time in his life that someone had asked about his past traumatic experiences.

When we asked patients what they preferred with regard to the treatment of their PTSD, most replied that they preferred a combination of individual and group treatments. Individual treatment was believed to be easier, more emotion-focused, safer, and more personal. Patients liked the attention they received in individual treatment and the fact that they could discuss more details. They expressed that they could learn more from an in-depth individual approach. Patients had experienced that they were better able to reflect during individual treatment. According to the patients, the ideal individual treatment would take place weekly with a female therapist: someone who is strong and, if possible, a "hands-on" expert. There should be trust and a certain connection between the therapist and the patient. Therapy should not necessarily be solution-focused; patients deemed it more important that the clinician listen, try to understand, confront, and keep on asking questions.

The patients also noted positive aspects of group therapy. These included the fact that group interaction can be very enjoyable, that theoretical knowledge can directly be applied, and that other patients often recognize problems and complaints. The patients pointed to the importance of closed groups and sufficient profundity. A risk of group therapy is that it can be too impersonal.

Analysis of the interviews showed that patients receiving trauma treatment not only want to confront the trauma, but to discuss daily hassles, like relationship and social problems. Some patients mentioned that medication should be part of the treatment. Others wanted to improve their social and coping skills during treatment. Since the patients believed that trauma treatment is hard and difficult, some of them preferred treatment in a clinical setting. Patients hoped that trauma treatment could give them a new start, teaching them how to enjoy life and feel connected with others. The patients mentioned that they were not fully aware of the benefits of trauma treatment and most of them did not know what kind of trauma treatment is possible. As one patient stated, "I don't know what kind of help you can get." 
Another important aspect patients mentioned was that they sometimes live in trauma-prone environments (e.g., with a violent partner). These patients felt that trauma treatment would not help as long as their surroundings did not change.

Although most patients favored integrated treatment of SUD and PTSD in which the person as a whole can be treated instead of only the addiction, some patients did have hesitations. They felt that trauma treatment would not change anything or they felt that there was no need for it, that life goes on. One patient stated: "I want to leave it behind me; I don't want to think about it." Another said:

"I don't know, I really don't know. It's so much...the enormous anxiety. On one hand, I think don't go into it; on the other hand, it keeps triggering me."

\section{Discussion}

In this qualitative study, we interviewed SUD/PTSD patients to learn more about their perceptions regarding their comorbidity. We then attempted to answer two research questions.

The first research question concerned the motives of SUD/PTSD patients for starting to use substances and continuing to do so. We noticed an apparent discrepancy in their answers. On the one hand, patients reported that PTSD was not linked with their very first substance use or with aggravation of their substance using pattern; instead, they mainly reported non-trauma-related reasons for starting to use (or using more) substances. On the other hand, all the patients reported self-medicating behavior and craving after trauma intrusions. They declared that coping motives were the most important reasons they used substances and that relapse often happened when they perceived no other means of coping with a difficult situation. These results are in agreement with earlier findings from Ouimette, Coolhart, Funderburk, Wade, and Brown (2007).

However, the self-medicating behavior of SUD/PTSD patients is not limited to coping with trauma-related stress. They also reported using substances to cope with daily hassles and general stressors and to feel more at ease in social environments. Previous researchers who compared the coping styles of SUD patients with and without PTSD found that SUD/PTSD patients are characterized by a maladaptive coping style (Gielen et al., 2015b; Ouimette, Finney, \& Moos, 1999; Staiger, Melville, Hides, Kambouropoulos, \& Lubman, 2009).

Our second research goal was to explore which treatment options SUD/PTSD patients found most feasible. None of the patients were being treated for PTSD and one patient even admitted that no one had ever asked about his traumatic past. This confirms our earlier findings about under diagnosis of PTSD in SUD patients (Gielen et al., 2012, 2014). We further found that patients are well aware of what 
kinds of treatment they prefer. Most SUD/PTSD patients favor simultaneous treatment of SUD and PTSD that combines individual and closed group treatment, and in which the person as a whole is treated (they judged a primary focus on SUD or PTSD to be insufficient). The patients further expressed a preference for female therapists. Ambulatory treatment was seen as a possibility but it should be possible to switch to in-patient treatment if necessary. Some patients were reluctant to start PTSD treatment, which is understandable in the light of their avoidance symptoms. A final important result was that SUD/PTSD patients knew very little about possible PTSD treatments. Clearly, these two factors (avoiding traumatic memories and lack of knowledge about treatment options) form barriers to implementing a standard integrated SUD/PTSD treatment for this particular patient group.

Although self-medicating behavior in SUD/PTSD patients seems obvious, it appears more appropriate to use a more complex etiological theory to explain the link between SUD and PTSD. The SUD/PTSD patients said that they did not start using drugs or alcohol because of their PTSD. However, the influence of PTSD on SUD is particularly important in maintaining any addictive behavior. PTSD has to be experienced as instrumental before it reinforces further drug use. More research on this issue is warranted. It would be particularly interesting to examine whether PTSD patients with and without SUD report differences in initial substance use and in the perceived function of the substance use.

Finally, a clear link exists between craving, relapse and PTSD symptoms. SUD/PTSD patients expect a whole-person approach when being treated for their PTSD. The integration of skills training and attention for patients who are fearful of PTSD treatment might further improve SUD/PTSD treatment results. 
Chapter 7

General

Discussion 
K's choice - Not an Addict

The deeper you stick it in your vein

The deeper the thoughts there's no more pain

I'm in heaven, I'm a god

I'm everywhere, I feel so hot 
Previous studies have documented a strong link between PTSD and SUD. Patients with this comorbidity bear the burden of two severe, mutually influencing, and often chronic disorders. Both SUD and PTSD might push a person into social isolation and secrecy. When such a person finally does seek help, treatment possibilities are limited.

When examining clinical guidelines and clinical research, there is a growing consensus that in case of SUD/PTSD comorbidity treatment should be offered, immediately, and in an integrated manner (Berenz \& Coffey, 2012; Kivlahan \& Kaysen, 2012; McCauley et al., 2012; Najavits \& Hien, 2013; Ruzek, Polusny, \& Abueg, 1998; Snoek, Wits, Meulders \& van de Mheen, 2012; van Dam et al., 2012). Why is it then that SUD treatment centers have not implemented this integrated treatment for comorbid SUD/PTSD (Ducharme, Knudsen, \& Roman, 2006; Killeen, Back, \& Brady, 2015)? And what is missing that might facilitate bridging the gap between SUD/PTSD research and clinical practice? In addition, the precise interaction between the two disorders is not clear yet. It is often suggested that substances are used to reduce the mental pain caused by PTSD, but it is also possible that the motives of SUD/PTSD patients to use substances are related to their inadequacy to cope with stress in general.

The central theme of the present dissertation concerned the interaction between SUD and PTSD. Three main aims were addressed in this thesis: (1) to study the prevalence and the vulnerability of patients with SUD/PTSD comorbidity (Chapters 2, 3, 5, and 6), (2) to study how the craving response of SUD/PTSD patients interacts with personalized trauma and stress cues (Chapters 3, 5, and 6), and (3) to examine why the implementation of integrated SUD/PTSD treatment has been unsuccessful (Chapters 2, 4,5 , and 6). The following sections provide an overview and discussion of the main findings; theoretical and clinical implications are put forward; limitations of the studies are discussed and suggestions for future research are given.

\section{Main findings}

PREVALENCE AND VULNERABILITY OF PATIENTS WITH SUD AND PTSD

The first aim of the dissertation was to examine both the prevalence and the vulnerability of patients with SUD and PTSD. The prevalence of PTSD and trauma exposure in SUD patients was compared with the prevalence of PTSD and trauma exposure in a "healthy" control-group using the same validated selfreport questionnaire in Chapter 2. More than one third of SUD patients scored positive on the PTSD questionnaire compared to one in ten of the individuals in the control group. And while both groups 
reported high rates of trauma exposure, this number was significantly higher in the SUD-patient group (97.4\% vs. $86.3 \%)$.

In the same study we investigated the vulnerability of SUD/PTSD patients. One finding was that SUD/PTSD patients share substantial similarities with SUD patients who experienced trauma but did not develop PTSD. Indeed, both groups did not differ in age, gender distribution, number of previous treatments, time in treatment, treatment outcome, primary substance, criminal past, financial debts, and relational status. Only two demographic factors were markedly different: SUD/PTSD patients were more often unemployed and had a lower educational level. Moreover, with regard to psychiatric comorbidity, SUD/PTSD patients were more likely to have depressive disorders and were more likely to have another axis I disorder compared to SUD/no PTSD patients.

Other results pointing to the vulnerability of SUD/PTSD patients are described in Chapter 3 . In this study, the sample consisted of SUD patients who had experienced trauma and who were sober or clean for some time. The participants had different levels of PTSD severity. The participants were exposed to narrative cues in the absence of in vivo drug cues. SUD/PTSD patients were found to crave more and to feel more tense in all conditions of this experiment as compared to SUD patients with lower levels of PTSD severity. Furthermore, SUD patients with high PTSD severity craved more after both stress and trauma exposure compared to neutral exposure. Thus, not only exposure to traumatic triggers, but also more general stress cues elicit strong craving in particularly SUD/PTSD patients and this makes them more vulnerable to relapse of addictive behavior after treatment.

The lack of adequate coping skills in SUD/PTSD patients was noted in the study described in Chapter 5. In this study, the sample consisted of SUD patients with previous trauma exposure and differing levels of PTSD severity. As PTSD severity predicted the coping outcome, it was concluded that SUD patients with PTSD have inadequate cognitive emotion regulation strategies. Specific for the SUD/PTSD patients was also the increased use of substances because of social motives, which was less frequently named as a motive in SUD patients with no/less severe PTSD and that SUD/PTSD patients perceived a strong link between relapse and traumatic intrusions.

The vulnerability of SUD/PTSD patients was also examined in a qualitative study described in Chapter 6. SUD/PTSD patients were interviewed and in the interviews, patients stressed that they did not only struggle with the consequences of past trauma and substance use, but that they also needed counseling for problems in their current social environment and relationships. Furthermore, the interviewed patients declared that they mostly used substances to cope with negative emotions or cognitions, implying the absence of adaptive coping skills. 
In sum, SUD/PTSD patients account for a substantial subgroup of patients. Their inadequacy to cope with social situations, negative affect, general stressors, and traumatic triggers puts them at risk for increased craving and relapse.

\section{CUED CRAVINGS TO TRAUMA AND STRESS CUES}

The second aim of this thesis was to study how the craving response of SUD/PTSD patients interacts with personalized trauma and stress cues. The participants of the experiment described in Chapter 3 were exposed to three auditory cues: a neutral script, their personalized trauma script, and a personalized stress script. SUD patients with high PTSD severity craved more and felt more tense throughout the experiment. Moreover, these patients craved significantly more after both stress and trauma cue exposure, compared to neutral script exposure.

The results described in Chapter 5 demonstrated that substances are abused to cope with the consequences of trauma, but equally to endure general stressors. However, this self-medicating behavior did not seem to be specific for SUD/PTSD patients. Indeed, SUD patients who experienced a past trauma but did not develop PTSD reported that they too use substances in response to trauma and stress. When SUD patients with and without PTSD were asked about their experiences of relapse as a

consequence of PTSD symptoms, PTSD severity did predict the outcome. SUD patients with high PTSD scores reported to relapse significantly more often as a consequence of their traumatic past.

The qualitative patient study (Chapter 6) showed that SUD/PTSD patients view craving, relapse, and trauma cues as interrelated, but also report that they use substances to cope with stress.

In sum, it appears that SUD/PTSD patients experience not solely a link between trauma cues and craving, but equally between stress cues and craving. Although, subjectively, SUD patients with low levels of PTSD severity also report to crave substances when remembering trauma or when experiencing stress, an experimental manipulation showed that this trauma/stress elicited craving is particularly strong in SUD/PTSD patients.

UNSUCCESFUL IMPLEMENTATION OF INTEGRATED SUD/PTSD TREATMENT

The third aim of this dissertation was to examine why the implementation of integrated SUD/PTSD treatment was not established. Chapter 2 revealed some important aspects with regard to this matter. It was found that structural screening of PTSD in SUD patients did not take place, resulting in a severe 
underdiagnosis of PTSD. Despite the fact that almost four out of ten SUD patients mentioned trauma exposure and one-fifth described PTSD symptoms during their intake interview, only in less than $5 \%$ of cases possible PTSD was reported by the clinicians who interviewed the patients during the intake procedure. When examining the final diagnosis in the patient files, this number further dropped; only $2.1 \%$ of the SUD patients was diagnosed with PTSD as compared to $36.6 \%$ when a validated questionnaire was used.

Addiction therapists, though well aware of the negative consequences of trauma on SUD, were not aware of the heightened prevalence score of PTSD (Chapter 4). It is conceivable that because of this underestimation PTSD treatment does not receive priority in SUD treatment and clinicians do not examine new treatment protocols, or specific diagnostic tools. Besides this underestimation, the interviewed clinicians reported that they were careful, even avoidant to discuss trauma with their SUD patients. This careful approach was fed by the perception that asking for and talking about trauma elicits craving, and possibly relapse. Furthermore, clinicians clearly stated that PTSD is not a focus in SUD treatment because the institution they work for does not support PTSD treatment during SUD treatment. In other words, clinicians neither receive training for PTSD diagnosis or treatment, nor are they allowed spending too much time on other problems besides SUD. As a result PTSD is not well assessed or treated during SUD treatment.

Chapter 5 describes some patient factors that might explain the unsuccessful implementation of the advised integrated treatment. Patients perceive that substance use is effective in diminishing PTSD symptoms, they feel that abstinence does not improve PTSD symptomatology, and they hold positive expectancies regarding the effect of the substance on their PTSD symptoms. It is therefore hardly surprising that SUD/PTSD patients are reluctant to start an integrated SUD/PTSD treatment.

Chapter 6 describes some last factors that might hinder successful implementation of integrated SUD/PTSD treatment. Since SUD/PTSD patients are anxious or reluctant to start PTSD treatment this might further discourage clinicians to implement PTSD treatment during SUD treatment. Also, patients are often not aware of possible PTSD treatments and this lack of knowledge might prevent them of discussing trauma treatment with their clinicians. The fact that all of the interviewed SUD/PTSD patients never received PTSD treatment confirms previous research findings that integrated SUD/PTSD treatment is not implemented.

Thus, it was found that the unsuccessful implementation integrated SUD/PTSD treatment is explained by a combination of factors: the severe underdiagnosis and underestimation of PTSD, the avoidance of both patients and clinicians, the absence of facilitation to diagnose and treat PTSD patients 
properly, the lack of knowledge of patients about PTSD treatment possibilities, and the efficiency of substances to diminish PTSD symptoms.

\section{Discussion of research findings}

PREVALENCE AND VULNERABILITY OF PATIENTS WITH SUD AND PTSD

It was found that $36.6 \%$ of SUD patients scored positive on PTSD and that almost every SUD patient had encountered at least one traumatic experience in his/her lifetime. These numbers are significantly lower in individuals without substance dependence. The results of some comparable studies (measuring current PTSD in a heterogeneous SUD population) are consistent with my prevalence data described in Chapter 2. In prior studies in SUD populations, current PTSD was found to be prevalent in $25.3-41 \%$ of cases (Bonin et al., 2000; Driessen et al., 2008; Ouimette et al., 2007; Read et al., 2004; Reynolds et al., 2005). Studies in SUD populations that assessed incidence of trauma exposure typically report percentages between 89\% and 95\% (Dansky, Saladin, Coffey, \& Brady, 1997; Farley, Golding, Young, Mulligan, \& Minkoff, 2004; Read et al., 2004; Reynolds et al., 2005), which also concurs with the present results in this dissertation.

With regard to the vulnerability of the SUD/PTSD patient subgroup we found that these patients are comparable to SUD patients with lower or no PTSD severity on most measured characteristics (age, gender distribution, number of previous treatments, time in treatment, treatment outcome, primary substance, criminal past, financial debts, relational status, and axis two comorbidity). There were however, seven characteristics that were specific for SUD/PTSD patients.

(1) SUD/PTSD patients are less educated and are more often unemployed, compared to SUD patients with no PTSD (Chapter 2). As educational level is a strong predictor for future opportunities on the labor market (Gesthuizen \& Scheepers, 2010) it is not surprising that SUD/PTSD patients are more often unemployed. There are different possible pathways that might explain the lower educational level of SUD/PTSD patients. Firstly, it is known that early trauma exposure affects the developing brain. (Klaassens, van Noorden, Giltay, van Pelt, van Veen, \& Zitman, 2009) and that PTSD patients have shown to experience more difficulties in executive functioning tasks (Aupperle, Melrose, Stein, \& Paulus, 2012; Leskin \& White, 2007). Therefore, they might experience more troubles during their primary and secondary education, affecting school performances, educational opportunities or school absenteeism. Secondly, 
peers and family also hold a crucial role in educational choice (Davis-Kean, 2005; Palmer, Hayek, Hossler, Jacob, Cummings, \& Kinzie, 2004) and when a child does not grow up in an aspirational environment chances are that this child ends up with a lower educational level compared to children who are raised in more stimulating environments (Ferry, Fouad, \& Smith, 2000; Flint, 1992; Sewell \& Shah, 1968). Thirdly, the symptomatology of both disorders further shows that education and employment are negatively affected by PTSD and SUD. The diminished interest or participation in important activities and the experience of significant impairment in important areas of functioning are two important PTSD symptoms that directly influence education and employment. SUD equally implies a failure to fulfill major role obligations at school or that important occupational activities are given up or reduced because of substance use (DSM IVTR). As unemployment is related with mental health problems (Paul \& Moser, 2009), poverty (Gallie, Paugam, \& Jacobs, 2003), and high society costs (Liem \& Rayman, 1982), it is clear that unemployment is an important vulnerability factor for SUD/PTSD patients.

(2) SUD patients with PTSD are more likely to have depressive disorders and are more likely to have another axis I disorder (Chapter 2). In a prospective study by Shalev and colleagues (1998) it was found that PTSD and major depression are independent consequences of trauma exposure and that, when comorbidity occurs, this is associated with more symptom severity. This again underlines the vulnerability of patients with comorbid SUD and PTSD. The fact that SUD/PTSD patients are more often diagnosed with depression and other axis I disorders might also be explained by the underdiagnosis or symptom entanglement of PTSD in SUD patients (Chapters 2 and 4). The finding that SUD clinicians are not trauma-focused, might lead to the underdiagnosis of PTSD (Chapter 4), but also to a misdiagnosis. As such, a patient who experiences traumarelated hallucinations or dissociative reactions can end up being diagnosed with a psychotic disorder.

(3) SUD/PTSD patients are more tensed in general (Chapter 3). This is not very surprising since PTSD is characterized by the experience of negative mood and increased arousal $\left(5^{\text {th }}\right.$ ed., DSM- 5 APA, 2013) and our results might thus merely reflect the symptomatology of PTSD. As chronic feelings of tension put individuals at risk for physical health problems (Allostatic load: McEwen, 1999), including diabetes, obesity, hypertension, heart disease, dermatological problems (Dallman et al., 2003; ), the increased and generalized level of tension in SUD/PTSD patients clearly demands immediate attention in treatment. 
(4) SUD/PTSD patients experience a higher level of craving in general (Chapter 3). As craving is an important predictor for relapse (Back et al., 2010; Schneekloth et al., 2012), this further suggests that SUD patients with high PTSD severity belong to an especially vulnerable subgroup of patients. It is possible that the increased level of tension precedes feelings of craving. The correlation analysis in Chapter 3 showed that tension ratings and craving ratings were highly correlated. In all three sessions baseline tension was highly correlated with craving ratings both before and after cue exposure. However, when observing the correlations between baseline craving rates and tension rates both before and after cue exposure, we found that the significance levels dropped, suggesting that tension precedes craving.

(5) SUD/PTSD patients abuse substances to feel more at ease in social environments (Chapter 5). This finding can be explained by the detachment, distrust, hyperarousal, and anxiety typically reported by PTSD patients. Our findings thus suggest that SUD/PTSD patients suffer discomfort in social company when abstinent. As a result they either avoid social interaction or endure the company while being intoxicated. In the long term, this probably leads to either social isolation or to selective interaction with other substance users.

(6) SUD patients with high PTSD scores report an increase in craving after exposure to both personalized stress cues and personalized trauma cues (Chapter 3) and report to relapse more often after being triggered by a traumatic cue (Chapters 5 and 6). The increased craving after trauma cue exposure is a replication of previous research by Coffey and colleagues $(2002,2010)$, Nosen and colleagues (2012), and Saladin and colleagues (2003) and our study adds that this effect endures in a more heterogeneous sample of patients who were abstinent for some time (mean abstinence was around 3 months) and even without concurrent exposure to an in vivo drug cue. Prior research also documented the existence of stress-induced craving in SUD patients (Fox, Bergquist, Hong, \& Sinha, 2007; Sinha, 2007; Sinha \& Li, 2007). The findings presented in Chapter 3 indicate that this effect is magnified when patients suffer from PTSD. When translating the results to the life of SUD/PTSD patients, this finding means that a significant increase in craving can occur just by being triggered to a past trauma or general stress cues, even in the absence of drugs or alcohol. These results further suggest how vulnerable SUD/PTSD patients are for relapse when PTSD is left untreated.

(7) SUD/PTSD patients have less adaptive cognitive emotion regulating strategies compared to SUD patients with lower PTSD scores (Chapter 5). The protective role of adequate coping skills has been established both in studies examining patients with PTSD (Bosmans \& van der Velden, 
2015), SUD (McConnell, Memetovic, \& Richardson, 2014), as in SUD patients who have been exposed to trauma (Anderson, Ramo, \& Brown, 2006; Bonar, Bohnert, Ilgen, Sanborn, \& Chermack, 2013; Brady, Tschann, Pach, Flores, \& Ozer, 2009). It is plausible that when an individual learns that substance use is an effective way to cope with trauma-related symptoms and to cope with daily stressors (Chapter 6), other coping skills are not developed, leaving this individual to rely on his/her established substance use coping mechanisms.

\section{CUED CRAVINGS TO TRAUMA AND STRESS CUES}

Our studies demonstrated a perceived and experimentally induced interrelatedness between on the one hand trauma and stress triggers and, on the other hand craving, substance use, or relapse (Chapters 3, 5 and 6). In two of our studies (Chapters 5 and 6) almost all SUD/PTSD patients reported substance use and craving after stress and trauma triggers. The experimental study, described in Chapter 2, confirmed that SUD patients with high PTSD severity craved more after exposure to both trauma and stress cues, relative to neutral cues. Thus, not only exposure to traumatic triggers, but also more general stress cues appear especially risky for relapse in patients with high PTSD scores. It is likely that patients who suffered from a past trauma and who learned that substance use can ease the pain never learned to cope effectively with negative feelings or cognitions and, therefore, rely on using substances when being confronted with trauma or stress cues. Indeed, the findings described in Chapter 5 show that SUD/PTSD patients have significantly lower cognitive emotion regulating strategies as compared to SUD patients with lower PTSD severity scores. Recently, Bosmans and van der Velden (2015) prospectively examined coping in 400 adults who had been exposed to a potentially traumatic event. They found that inadequate coping predicted the development of subsequent PTSD symptoms, but PTSD symptoms did not predict coping. They did, however, not measure coping before the traumatic incident. So, inadequate coping skills seem to form an important factor in the relation between trauma exposure and PTSD (Bosmans \& van der Velden, 2015), between high life stress/trauma and substance use (Anderson et al., 2006; Brady et al., 2009), and between PTSD and SUD (Asberg \& Renk, 2012; Ullman, Relyea, Peter-Hagena, \& Vasquez, 2013).

UNSUCCESFUL IMPLEMENTATION OF INTEGRATED SUD/PTSD TREATMENT 
Results of several studies provided more insight into why integrated SUD/PTSD treatment is not implemented in SUD treatment centers. Three barriers hindering implementation of integrated SUD/PTSD treatment can be identified: (1) obstacles related to the clinician, (2) obstacles related to the institution, and (3) obstacles related to the patients. Implementation of integrated SUD/PTSD treatment is likely to fail if not all three barriers are removed.

On the level of the clinicians two main obstacles were detected. Firstly, PTSD remains largely undetected in SUD treatment centers (Chapter 2). This happens as a result of not using standardized PTSD questionnaires and as a result of not routinely screening for PTSD during different stages of the treatment process. Consequently, PTSD prevalence is severely underestimated, leading to undertreatment and not being familiar with integrated treatment protocols, specific guidelines, or useful diagnostic tools. Secondly, clinicians seemed afraid that discussing trauma with the patient may put him/her in jeopardy for a (re-)lapse (Chapter 4). This same obstacle has been identified in previous studies as well (Back, Waldrop, \& Brady, 2009; Najavits, 2002). Although our and previous studies suggest that trauma cues will increase the patient's craving for alcohol or drugs (Coffey et al., 2002; Saladin et al., 2003, Chapter 3), this does not compromise treatment outcome. Indeed, as mentioned above, there are several studies showing better overall treatment outcome with integrated SUD/PTSD treatment, compared to SUD treatment only (Torchalla et al., 2012; van Dam et al., 2012). Therapists appear wary not to harm their patients, as they should, but a good therapist should not be so cautious as not to provide treatment at all.

On the level of the institution it is found that lack of time, money, and expertise are main hurdles that explain the unsuccessful implementation of the integrated treatment (Chapter 4). SUD treatment centers do not invest in the integrated approach of comorbid SUD/PTSD. The results described in Chapter 4 suggest that clinicians are willing to implement the integrated treatment if facilitated by the institution they work for.

On the patient level, three main obstacles are identified: (1) patients are anxious/avoidant to start PTSD treatment, possibly enhancing the clinicians' reluctance to discuss trauma, (2) patients are not aware of the treatment possibilities, which might inhibit them to discuss their PTSD symptoms with their SUD clinicians, and (3) SUD/PTSD patients reported in several of our studies that substance use is an effective way to cope with PTSD symptoms. This last point deserves some elaboration. The results from the studies described in Chapters 3, 5, and 6 clearly indicate that SUD/PTSD patients crave and use substances when being confronted with the consequences of their traumatic past $190.9 \%$ of SUD/PTSD patients reported using substances after intrusions; Chapter 5) and, similarly, when being confronted 
with daily stressors. Although the patients are aware that the experienced positive effects of drugs and alcohol are superficial and temporary, they do experience them as effective means to dampen negative emotions or cognitions $(90 \%$ of SUD/PTSD patients reported that substance use is effective in diminishing an intrusion).

\section{Theoretical implications}

Based on the results presented in the previous chapters a working model is proposed explaining the symptom interplay between PTSD and SUD. The key concept within this model is inadequate coping.

Individuals who develop comorbid SUD/PTSD commonly experienced trauma at an early age (see e.g. Chapter 2). In most of these patients trauma is not a single isolated occurrence; on the contrary, SUD/PTSD patients typically report experiencing more than one trauma throughout their life (Chapter 2). As is illustrated in Figure 1, this early and extensive trauma exposure can lead to the development of PTSD. Trauma exposure can also lead to the development of other disorders, such as depressive disorders (Chapter 2) or borderline personality disorder (Pagura, Stein, Bolton, Cox, Grant, \& Sareen, 2010; Spinhoven, Penninx, van Hemert, de Rooij, \& Elzinga, 2014). First substance use happens, seemingly independent of the endured trauma (Chapter 6). That is, patients report that they do not take their drink (or get stoned or high) for the first time because of trauma or as a consequence of stress. Rather, first substance use is provoked by curiosity and seen as part of experimenting in one's youth. This impulsive need to experiment however may still be a consequence of trauma exposure. There is ample evidence that early life trauma exposure negatively affects the developing brain. Normal development of the medial prefrontal cortex is impeded, the functioning of the amygdala is attenuated, the volume of the hippocampus is reduced, and the hypothalamic-pituitary-adrenal axis becomes hyperreactive (Shin, Rauch, \& Pitman, 2006; Teicher, Andersen, Polcan, Navalta, \& Kim, 2003; Vermetten \& Lanius, 2012). Addictive behavior is associated with compromised functioning of the medial prefrontal cortex (involved in cognitive and executive control, emotional regulation, memory consolidation (Alexander \& Brown, 2011; Etkin, Egner, \& Kalisch, 2011; Euston, Gruber, \& McNaughton, 2012) and with a reduced volume of the hippocampus (involved in stress regulation and declarative memory processing; see Cheetham, Allen, Whittle, Simmons, Yücel, \& Lubman, 2012). Early life trauma exposure might thus put some individuals at an increased risk for substance use (Langeland, 2009).

A traumatized individual, through experimenting with alcohol or drugs, learns that substances lead to a decline in potential PTSD symptoms and that substance use is equally effective to cope with 
general stress (Chapters 3 and 5). The studies described in the current dissertation show that patients with comorbid SUD/PTSD indeed perceive substance use to be reinforcing; that is, it is believed to be an extremely effective way to deal with general stress and with PTSD symptoms (Chapters 5 and 6). Because of the positive and negative reinforcing characteristics of an addictive substance, substance use culminates into abuse and ultimately addiction. Feelings of stress and specific PTSD symptoms such as intrusive trauma memories become powerful predictors of substance use and - as argued in the General Introduction of this dissertation - through Pavlovian learning become incentive cues triggering intense cravings. This craving then further fuels instrumental substance use. Importantly then, PTSD is not seen as a direct cause of substance abuse or SUD but as a prominent risk factor for developing an addiction. This sequence is shown in Figure 1.

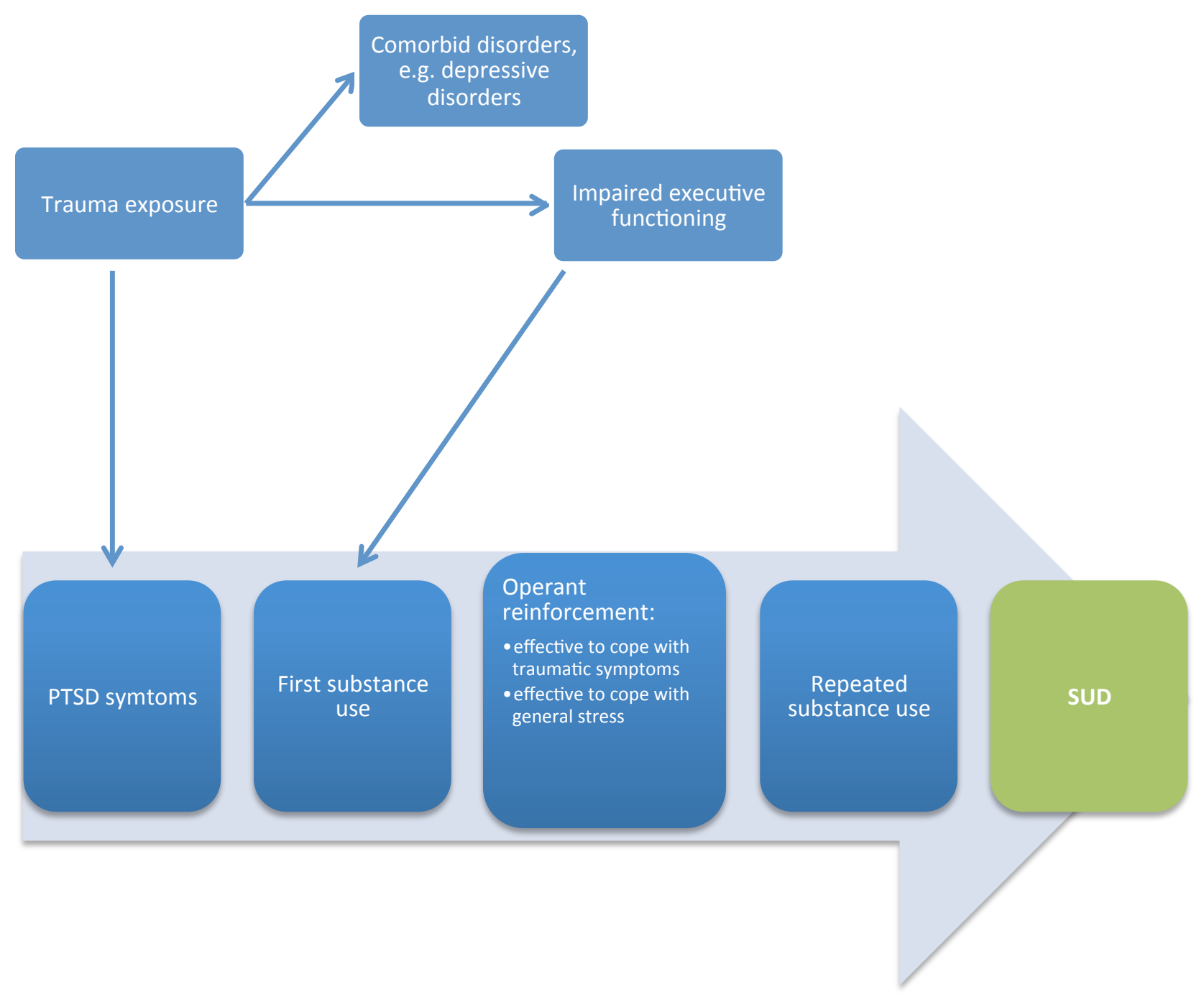

Figure 1. Presumed influence of trauma exposure on the development of SUD. 
Throughout the described studies it was found that SUD/PTSD patients have inadequate coping skills (Chapters 3, 5 and 6) and it was suggested that treatment should focus on coping skills training as part of a stabilization phase in these patients. But this still leaves us with the question whether SUD/PTSD patients have lost their capacity to adequately cope with stress somewhere during their life (e.g. after trauma exposure or after the development of PTSD or SUD), or whether these inadequate coping skills are inherent to the person. As the medial prefrontal cortex is involved in coping (HarmonJones, E., Sigelman, Bohlig, \& Harmon-Jones, C., 2003; Maier \& Watkins, 2010) and this brain structure shows dissimilarities in individuals who are traumatized at an early age (Shin et al., 2006; Teicher et al., 2003; Vermetten \& Lanius, 2012) it is arguable that the neurobiological changes induced by early life trauma exposure causes impairments in executive functioning, thereby affecting coping capacities and making them more vulnerable for developing PTSD and SUD. The inadequate coping skills can thus be seen as a third factor or a shared vulnerability factor that influences both PTSD and SUD.

From our findings it can be deduced that SUD/PTSD patients experience a high level of baseline tension, and these SUD/PTSD patients experience a further increase in tension as a reaction on daily stressors, social situations and traumatic triggers. As they have no adequate skills available to cope with this increased tension and since SUD/PTSD patients learned that substance use is an effective way to cope with stress and trauma triggers, craving and/or subsequent substance use follows. After substance use a temporary relief of negative emotions is experienced and the substance is intrinsically reinforced. As a consequence of this learning experience, there is no need for the SUD/PTSD patient to learn alternative and healthier coping strategies. Why should he/she? As is described in Chapters 5 and 6 , the substance use is perceived as a highly effective way of coping. So, the circle closes and new stressors will be dealt with in exactly the same way, further reinforcing the connection between stress/trauma triggers and substance use. From this it can be assumed that coping skills have little chance to improve in SUD/PTSD patients. This reinforcing chain of events is illustrated in Figure 2. 


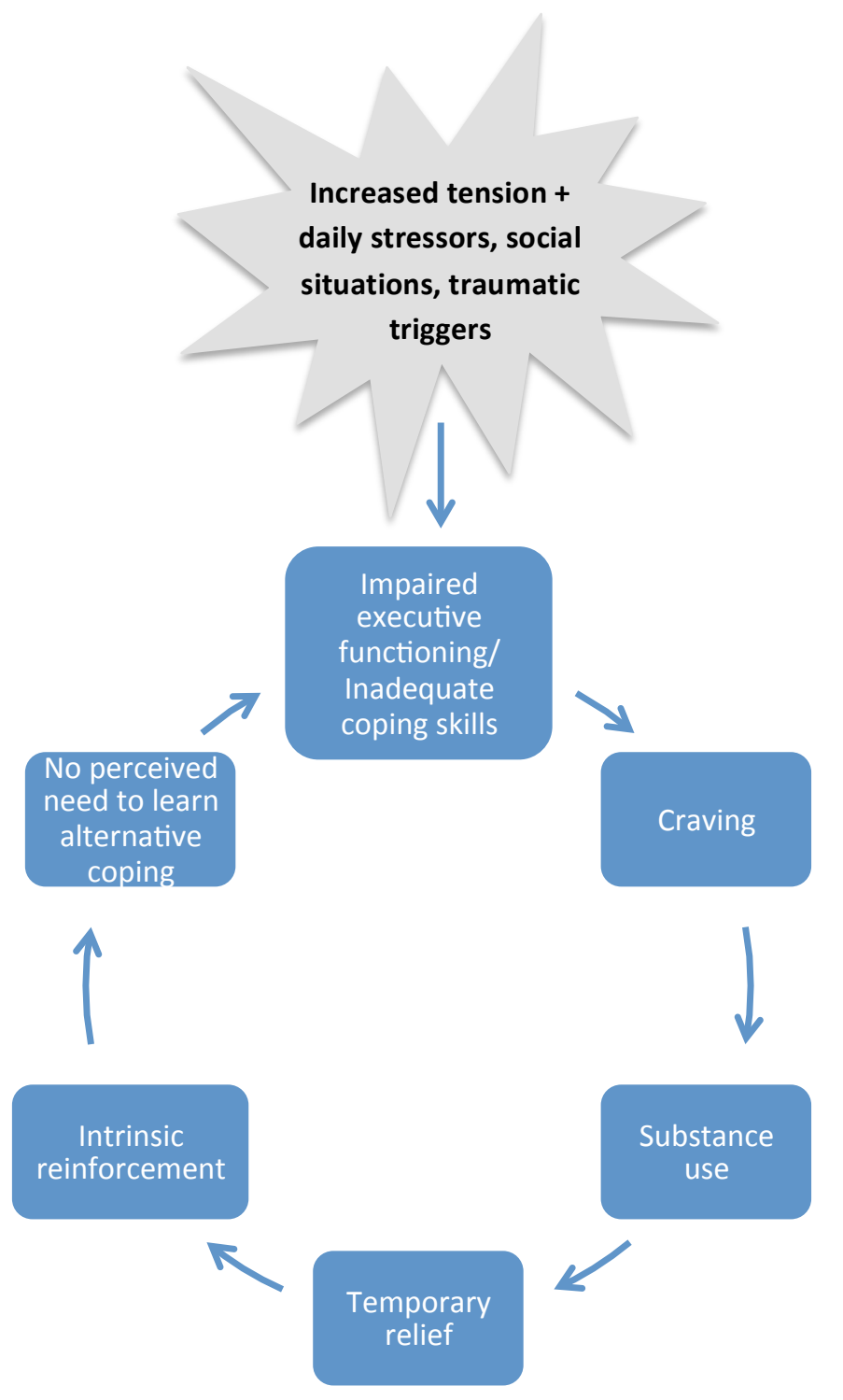

Figure 2. Vicious cycle starting from the inadequacy of coping skills.

One of the main symptoms of PTSD is avoidance. It is clear that in SUD/PTSD patients, avoidance plays a major role. Substance use is a way to avoid and/or escape from intrusions and emotional pain (Chapters 5 and 6 , the self-medication hypothesis). And it is precisely this avoidant coping style that in the longer term increases the risk that PTSD develops into a chronic and long-lasting disorder in SUD patients. Effective treatment of PTSD requires some form of exposure to the memory of trauma (Jaycox, Foa, \& Morral, 1998).

Although the presented theoretical model postulates that trauma exposure happens before SUD, in some patients trauma can occur after the development of SUD. Exploratory analysis on the data described in Chapter 2 indeed revealed that $18.5 \%$ of SUD/PTSD patients reported they were exposed to a traumatic event after the start of their addictive behaviour, but that in the great majority of cases 
(81.5\%) trauma exposure happened before their substance abuse (mean age of 13.79 vs. mean age of 23.96). The presented theoretical model is applicable irrespective of the chronological order of events; I hypothesize that the intrinsic reinforcing nature of a substance is of such strength that it overrules previously learned coping mechanisms. This of course merits further investigation.

Taken together, the SUD/PTSD symptom interplay is complex and cannot be grasped into only one of the existing models.

\section{Recommendations for clinical practice}

Based on the severe underestimation of PTSD in SUD patients (Chapter 4) and its consequences for clinical practice (Chapters 2 and 4), it is relevant to inform SUD treatment centres/clinicians about the finding that one in three of their SUD patients are likely to suffer from PTSD (Chapter 2).

Furthermore, since clinicians are not familiar with the SUD/PTSD guidelines (Chapter 4), there is a missing link in the process between the publication and the actual implementation of the guideline. In an attachment to the Dutch guideline on the treatment of comorbid SUD and anxiety disorders (Snoek, Wits, Meulders, \& van de Mheen, 2012) a four-page implementation plan is incorporated. This plan contains critical questions for situation analysis on an organizational level, a summary of advancing and impeding factors, a summary of possible implementation strategies, and a checklist of ingredients for the step-by-step implementation plan. Although this implementation plan might be very useful when SUD organizations decide to start implementing an integrated treatment for SUD/PTSD, it is of little use when this information does not reach the SUD treatment center/clinician. Something or rather someone is missing, a person that brings this - and other guidelines - under the attention of SUD treatment centers/clinicians. This can be a function for the developers of guidelines. An alternative, or better an addition, is that SUD treatment centers assign one of their clinical staff members with the function to monitor new guideline developments and that this employee is held responsible for the implementation of the new guideline in his/ her work setting.

To minimalize the risk for future under diagnosis -and therefore under treatment- of PTSD in SUD patients (Chapters 2 and 4), and to optimize screening and accurate diagnosis of trauma and PTSD among SUD patients, clinicians should also be informed about how and when to screen for PTSD. The results of this dissertation emphasise the need to identify the SUD/PTSD patients as a substantial and important subgroup and to systematically screen for PTSD in all SUD patients at intake. For this purpose, validated PTSD assessment tools should be made available in addiction treatment facilities. As is 
described in the recently developed guidelines, clinicians ought to diagnose PTSD by means of clinical interviews and clinical judgment (Snoek et al., 2012). Our results draw attention to the fact that, with regard to the diagnosis of PTSD, SUD treatment facilities should not solely rely on the opinions of clinicians and interviewers. Clinical judgement is prone to serious bias and therefore validated assessment tools should be used (Dawes, Faust, \& Meehl, 1989; Garb, 2005). Questionnaires like the JPTSD (modified version of the PC-PTSD) and the SRIP have shown to be efficient and validated measures in SUD populations (Kok, de Haan, van der Velden, van der Meer, Najavits, \& de Jong, 2013; van Dam, Ehring, Vedel \& Emmelkamp, 2010). The J-PTSD consists of only 4 questions and is an ideal instrument for a first screening. The SRIP can be administered as a second self-report measure when a patient scores positive on the J-PTSD.

My results indicate that SUD clinicians are not properly trained in diagnosing PTSD (Chapter 4). We acknowledge that reliable PTSD diagnosis requires specific clinical skills and training is necessary. The golden standard for diagnosing PTSD is to interview the patient with the Clinical-Administered PTSD Scale for DSM-5 (CAPS-5; Weathers, Blake, Schnurr, Kaloupek, Marx, \& Keane, 2013). Special one-day CAPS-5 trainings are offered for psychologists and psychiatrists to enable these clinicians in diagnosing PTSD with the CAPS-5. Training every SUD clinician in this interview is not only expensive, but also excessive; it probably suffices to train one or two clinicians in every SUD treatment center in using the CAPS-5.

My studies show that the efficacy of addiction treatment programs would likely benefit from including some form of PTSD treatment for SUD patients with clear (and sometimes debilitating) trauma exposure. SUD treatment centres should invest in the evidence based integrated approach of comorbid SUD/PTSD. Clinicians should be educated and trained to provide this integrated treatment. Integrating a trauma-focused component in SUD treatment seems fit, even for patients with lower levels of PTSD severity. Psycho-education about the link between SUD and past trauma can be a first and non-invasive step towards a better understanding of symptom interplay. Furthermore, although almost all SUD patients with a traumatic past reported the effective use of substances to cope with intrusions, the inadequacy of coping skills was very specific for SUD patients with high PTSD severity, implying that SUD/PTSD patients should receive extensive coping skill training, probably even before a past-focused therapy takes place. This is exactly what is suggested in a phase-oriented treatment for complex PTSD (Cloitre, Courtois, Charuvastra, Carapezza, Stolbach, \& Green, 2011). The first phase of this treatment consists of improving coping skills and ameliorating emotion regulation strategies (see Cloitre, et al., 2011). My results further show that patients are well aware of what kind of treatment they prefer. Most 
SUD/PTSD patients favour a simultaneous treatment of SUD and PTSD, combining individual and closed group treatment, in which the person as a whole is treated (a focus primarily on SUD or PTSD was judged to be insufficient). Following the stepped care model, the combined treatment can be done on an ambulatory basis, although more severe cases may need a clinical treatment (Snoek et al., 2012). Treatment of PTSD is possible even for non-abstinent patients. Nevertheless, certain control is needed so that patients can follow the therapy sessions without being under the influence of substances (Snoek et al., 2012). Multimorbidity or triple diagnosis is obviously a more complex problem but combined treatment seems useful in these patients (Snoek et al., 2012). Some patients were reluctant to start PTSD treatment which is understandable in the light of their avoidance symptoms. With this information in mind, attention for patients who are fearful of PTSD treatment might further improve SUD/PTSD treatment results. The well investigated Seeking Safety protocol can be used in patients where pastfocused trauma therapy is not possible (Hien et al., 2009; van Dam et al., 2012). This treatment can start even before detoxification and is often prolonged in nature (Snoek et al., 2012).

SUD/PTSD patients are not familiar with treatment options (Chapter 6) and factors as shame or anxiety might further inhibit them to discuss trauma and trauma treatment options with their SUD caregivers. When patients are informed about the SUD/PTSD comorbidity on a very general way this might remove patient barriers to discuss trauma during SUD treatment. One possible way in achieving this is to display PTSD brochures or posters in the waiting rooms of SUD treatment centres. A patient brochure about comorbid SUD and anxiety disorders is included in the Dutch guideline and contains psycho-education concerning diagnosis and treatment possibilities.

\section{Recommendations for the future}

Based on the results, the methodological remarks and the proposed theoretical model, some recommendations for future research can be offered. As will be noted in the amount of proposed new studies, this dissertation surely does not offer answers to all aspects of the SUD/PTSD comorbidity, rather it seems to raise more new questions than it answers.

A first recommendation is to study the prevalence of SUD in large sample of patients who are treated for PTSD using the same questionnaire as we did in our prevalence study and to compare this prevalence rate with SUD in a control group without PTSD. Part of this study can include the measurement of comorbid disorders, such as depressive disorders, so this specific comorbid disorder can be compared between a sample of PTSD patients vs. a sample of SUD/PTSD patients. Besides this, it 
is also relevant to know whether PTSD patients differ from SUD/PTSD patients with regard to coping skills, motives for first and possibly continued substance use. Other research questions that merit attention is how well PTSD treatment centers screen for SUD and what the perceptions of PTSD clinicians are about integrated SUD/PTSD treatment?

Another subject worthwhile exploring is the role of gender. Our studies did not take this question into account and women were clearly underrepresented in our samples. So, what is the role of gender with regard to PTSD prevalence in SUD samples, PTSD severity, craving responses, and coping skills? Since previous studies have shown that women display a different craving pattern than men (Fattore, Altea, \& Fratta, 2008) it would be interesting to further explore a possible gender difference using a cue reactivity paradigm in future studies.

Our results lend themselves to suggestions for intervention studies. As we found that SUD/PTSD patients are reserved, even anxious to start PTSD treatment, it is well worth to investigate whether a specific intervention, such as psycho-education, has an effect on this reserve and whether SUD/PTSD patients would be more inclined to start PTSD treatment after this psycho-education. Another possible intervention to be studied in SUD/PTSD samples is a social skills training. As SUD/PTSD patients reported that social motives are an important motivating factor to continue substance use, we might expect that when focusing on social skills specifically, this would have a positive influence on SUD related symptoms. A more general coping skills training is thought to be very suitable for SUD/PTSD patients. Protocolled stabilization programs like "Vroeger en Verder" (Dorrepaal et al., 2012) and Seeking Safety (Najavits, Weiss, Shaw, \& Muenz, 1998) include exercises to improve coping skills. The efficacy of Seeking Safety has been researched in more than 30 studies (see http://www.treatment-innovations.org/evid-allstudies-ss.html), but "Vroeger en Verder" has never been studied in SUD/PTSD patients.

\section{Conclusions}

Five main conclusions can be drawn from the research in this thesis. Firstly, more than one third of treatment seeking SUD patients suffers currently from the consequences of a past traumatic event and nearly all SUD patients are victims of one or more previous exposures to trauma.

Secondly, SUD/PTSD patients belong to an especially vulnerable subgroup of SUD patients; they are more often unemployed and depressed, they experience a higher level of overall tension and craving, and they are more prone to use substances in social situations. 
Thirdly, SUD/PTSD patients experience higher levels of craving when they are exposed to stress or trauma triggers and this makes them more vulnerable for relapse.

Fourthly, factors that impede the implementation of integrated SUD/PTSD treatment are: clinicians are unaware of the high prevalence of the comorbidity, SUD/PTSD clinicians and patients are reserved to discuss trauma, SUD facilities do not facilitate integrated treatment, SUD/PTSD patients are unfamiliar with treatment possibilities, and SUD/PTSD patients perceive substance use as a reinforcing way to cope with stress and traumatic triggers.

And finally, as the inadequacy of coping skills is very specific for SUD/PTSD patients and as this characteristic is seen as an important factor in the maintenance of comorbid SUD/PTSD, integrated SUD/PTSD treatment should include both stabilization (focusing on improving coping skills) and confrontation (focusing on exposure to the traumatic memory). 
Summary 
Previous studies have documented a strong link between PTSD and SUD. Patients with this comorbidity bear the burden of two severe, mutually influencing, and often chronic disorders. Both SUD and PTSD might push a person into social isolation and secrecy. When such a person finally does seek help, treatment possibilities are limited.

When examining clinical guidelines and clinical research, there is a growing consensus that in case of SUD/PTSD comorbidity treatment should be offered, immediately, and in an integrated manner. Why is it then that SUD treatment centers have not implemented this integrated treatment for comorbid SUD/PTSD? And what is missing that might facilitate bridging the gap between SUD/PTSD research and clinical practice? In addition, the precise interaction between the two disorders is not clear yet. It is often suggested that substances are used to reduce the mental pain caused by PTSD, but it is also possible that the motives of SUD/PTSD patients to use substances are related to their inadequacy to cope with stress in general.

This dissertation focuses on the interaction between SUD and PTSD. Three main aims are addressed in this thesis: (1) to study the prevalence and the vulnerability of patients with SUD/PTSD comorbidity, (2) to study how the craving response of SUD/PTSD patients interacts with personalized trauma and stress cues, and (3) to examine why the implementation of integrated SUD/PTSD treatment has been unsuccessful. To address these three aims I conducted five studies (Chapters 2 -7). Main findings that are reported in the chapters of this dissertation will be summarized.

Chapter 2 reports on a study with three objectives. Firstly, the prevalence of post-traumatic stress disorder (PTSD) and trauma exposure was compared between individuals with and without substance use disorder (SUD). Secondly, we compared self-rating of PTSD and clinical judgment. Thirdly, an analysis of the characteristics of SUD/PTSD patients was performed. The sample consisted of 423 patients with SUD and 206 healthy controls. All individuals were screened on PTSD using the self-rating inventory for PTSD. Significantly higher numbers of PTSD and trauma exposure were found in the SUD group (resp. 36.6 and 97.4\%). Furthermore, PTSD went frequently unnoticed when relying on clinical judgment alone. With regard to the characteristics, it was found that patients with SUD/ PTSD were significantly more often unemployed and had a lower educational level. Axis I comorbidity and especially depressive disorders were more common in the SUD/PTSD group. From these findings it is concluded that patients with SUD/PTSD are a substantial and vulnerable subgroup in addiction treatment facilities and that a systematic screening for PTSD is required.

Previous research shows that patients with substance use disorder (SUD) and post-traumatic stress disorder (PTSD) experience more craving for a substance after exposure to their personal trauma 
cues compared to neutral cues. However, it is not clear if their substance cravings are triggered by specifically trauma cues or by stress cues in general and whether the level of PTSD matters To examine the impact of trauma and stress cues on substance craving and tension in SUD/PTSD patients, we conducted an experimental study which is described in Chapter 3. Seventy-four patients attended three exposure sessions in which the patient was exposed to one tape-recorded script a neutral script, a stress script and a trauma script. Craving and tension were measured before and after each cue exposure. The findings show that patients high in PTSD severity craved more after stress and trauma script exposure, compared to exposure to the neutral script and to patients low in PTSD severity. These findings suggest that SUD/PTSD patients are not only more vulnerable to trauma cues, but also to more general stress cues.

Despite empirical support, integrated treatment of Substance Use Disorder (SUD) and Posttraumatic Stress Disorder (PTSD) is not sufficiently implemented in SUD facilities. To understand the reasons for this gap between theory and practice, we conducted a qualitative study on the views of clinicians with regard to the diagnosis and treatment of PTSD in SUD patients (presented in Chapter 4). An independent interviewer interviewed fourteen staff members of different wards of an addiction care facility. Despite acknowledging adverse consequences of trauma exposure on SUD, severe underdiagnosis of PTSD was mentioned and treatment of PTSD during SUD treatment was not supported. Obstacles related to the underestimation of PTSD among SUD patients and to the perceptions of SUD clinicians concerning the treatment of comorbid SUD/PTSD are reported. It is concluded that SUD treatment centres should train their clinicians to enable them to provide for integrated treatment of SUD/PTSD.

In Chapter 5 the perceptions of SUD/PTSD patients about the co-occurrence and the symptom interplay of the two disorders are addressed. Seventy-two SUD patients with differing levels of PTSD severity filled out a survey and three self-report questionnaires pertaining to their perceptions. Regression analysis was used to test whether PTSD severity accounts for possible differences between perceptions about the link between SUD and consequences of trauma exposure. Patients perceive that substance use is effective in diminishing PTSD symptoms, they feel that abstinence does not improve PTSD symptomatology, and they hold positive expectancies regarding the effect of the substance on their PTSD symptoms. These results suggest that it is hardly surprising that SUD/PTSD patients are reluctant to start an integrated SUD/PTSD treatment. Treatment implications are discussed.

Finally, in the qualitative study presented in Chapter 6, patients with comorbid substance use disorder (SUD) and post-traumatic stress disorder (PTSD) were interviewed on their ideas about the link 
between SUD and PTSD. Although they clearly reported self-medication, they also gave a more complex description of how they believe their PTSD influences their SUD. The results suggest that SUD/PTSD patients believe they did not start using substances because of their experienced traumas or PTSD, but that PTSD symptoms are nonetheless important in the maintenance of their addictions. A clear link exists between craving, relapse, and PTSD symptoms. SUD/PTSD patients would prefer a "whole-person approach" when being treated for their PTSD. It is suggested that the integration of skills training and attention for patients who are fearful of PTSD treatment might improve SUD/PTSD treatment results.

The thesis is concluded with Chapter $\mathbf{7}$ where the above reported findings are discussed in light of the three main goals that are described in chapter 1 . A working model is proposed explaining the SUD/PTSD symptom interplay and recommendations for clinical practice and for future research are suggested. To recapitulate, the studies demonstrate that nearly all SUD patients have been exposed to previous trauma and that one in three SUD patients still suffers from the consequences of this past experience. The findings further stress the vulnerability of SUD/PTSD patients; SUD/PTSD patients show, for instance, increased cravings both after stress and after trauma cues. The results of the research conducted for this thesis underline the importance to address coping skills in the integrated treatment of SUD/PTSD and emphasize that factors that impede the implementation of integrated SUD/PTSD treatment should be taken into account by SUD facilities. 
References 
Amaro, H., Chernoff, M., Brown, V., Arévalo, S., \& Gatz, M. (2007). Does integrated trauma-informed substance abuse treatment increase treatment retention? Journal of Community Psychology, 35(7), 845-862.

American Psychiatric Association. (2000). Diagnostic and statistical manual of mental disorders (4th ed., text rev.). Washington, DC: Author.

American Psychiatric Association. (2013). Diagnostic and statistical manual of mental disorders (5th ed.). Washington, DC: Author.

Anderson, K. G., Ramo, D. E., \& Brown, S. A. (2006). Life stress, coping and comorbid youth: An examination of the stress-vulnerability model for substance relapse. Journal of Psychoactive Drugs, 38(3), 255-262.

Asberg, K., \& Renk, K. (2012). Substance use coping as a mediator of the relationship between trauma symptoms and substance use consequences among incarcerated females with childhood sexual abuse histories. Substance use \& misuse, 47(7), 799-808.

Aupperle, R. L., Melrose, A. J., Stein, M. B., \& Paulus, M. P. (2012). Executive function and PTSD: disengaging from trauma. Neuropharmacology, 62(2), 686-694.

Avants, S. K., Margolin, A., Kosten, T. R., \& Cooney, N. L. (1995). Differences between responders and nonresponders to cocaine cues in the laboratory. Addictive Behaviors, 20(2), 215-224.

Back, S. E., Brady, K. T., Sonne, S. C., \& Verduin, M. L. (2006). Symptom improvement in co-occurring PTSD and alcohol dependence. The Journal of Nervous and Mental Disease, 194(9), 690-696.

Back, S. E., Hartwell, K., DeSantis, S. M., Saladin, M., McRae-Clark, A. L., Price, K. L., ... \& Brady, K. T. (2010). Reactivity to laboratory stress provocation predicts relapse to cocaine. Drug and Alcohol Dependence, 106(1), 21-27.

Back, S. E., Sonne, S. C., Killeen, T., Dansky, B. S., \& Brady, K. T. (2003). Comparative profiles of women with PTSD and comorbid cocaine or alcohol dependence. The American Journal of Drug and Alcohol Abuse, 29(1), 169-189.

Back, S.E., Waldrop, A.E., Brady, K.T., \& Hien, D. (2006). Evidence-based time-limited treatment of co-occurring substance-use disorders and civilian-related posttraumatic stress disorder. Brief Treatment and Crisis Intervention, 6, 283-294.

Back, S.E., Waldrop, A.E., \& Brady, K.T. (2009). Treatment challenges associated with comorbid substance use and posttraumatic stress disorder: clinicians' perspectives. The American Journal on Addictions, 18, 15-20.

Berenz, E. C., \& Coffey, S. F. (2012). Treatment of co-occurring posttraumatic stress disorder and substance use disorders. Current Psychiatry Reports, 14(5), 469-477.

Blake, D. D., Weathers, F. W., Nagy, L. M., Kaloupek, D. G., Charney, D. S., \& Keane, T. M. (1995). Clinician administered PTSD scale for DSM-IV (CAPS-DX). National Center for Posttraumatic Stress Disorder, Behavioral Science Division, Boston VA Medical Center, Boston, MA.

Bonar, E. E., Bohnert, A. S., Ilgen, M. A., Sanborn, M. L., \& Chermack, S. T. (2013). Physical assault victimization and coping among adults in residential substance use disorder treatment. Violence and Victims, 28(5), 849. 
Bonin, M. F., Norton, G. R., Asmundson, G. J., Dicurzio, S., \& Pidlubney, S. (2000). Drinking away the hurt: The nature and prevalence of PTSD in substance abuse patients attending a community-based treatment program. Journal of Behavior Therapy and Experimental Psychiatry, 31(1), 55-66. doi:10.1016/S00057916(00)00008-2

Bosmans, M. W., \& van der Velden, P. G. (2015). Longitudinal interplay between posttraumatic stress symptoms and coping self-efficacy: A four-wave prospective study. Social Science \& Medicine, 134, 23-29.

Brady, K. T., Back, S. E., \& Coffey, S. F. (2004). Substance abuse and posttraumatic stress disorder. Current Directions in Psychological Science, 13(5), 206-209.

Brady, K. T., Back, S. E., Waldrop, A. E., McRae, A. L., Anton, R. F., Upadhyaya, H. P., ... \& Randall, P. K. (2006). Cold pressor task reactivity: Predictors of alcohol use among alcohol-dependent individuals with and without comorbid posttraumatic stress disorder. Alcoholism: Clinical and Experimental Research, 30(6), 938-946.

Brady, S. S., Tschann, J. M., Pasch, L. A., Flores, E., \& Ozer, E. J. (2009). Cognitive coping moderates the association between violent victimization by peers and substance use among adolescents. Journal of Pediatric Psychology, 34(3), 304-310.

Breslau, N., Davis, G. C., \& Schultz, L. R. (2003). Posttraumatic stress disorder and the incidence of nicotine, alcohol, and other drug disorders in persons who have experienced trauma. Archives of General Psychiatry, 60(3), 289-294.

Bronner, M. B., Peek, N., Vries, M. D., Bronner, A. E., Last, B. F., \& Grootenhuis, M. A. (2009). A community-based survey of posttraumatic stress disorder in the Netherlands. Journal of Traumatic Stress, 22(1), 74-78.

Brown, P. J., Stout, R. L., \& Gannon-Rowley, J. (1998). Substance use disorder-PTSD comorbidity: Patients' perceptions of symptom interplay and treatment issues. Journal of Substance Abuse Treatment, 15(5), 445-448. doi:10.1016/s0740-5472(97)00286-9

Cheetham, A., Allen, N. B., Whittle, S., Simmons, J. G., Yücel, M., \& Lubman, D. I. (2012). Orbitofrontal volumes in early adolescence predict initiation of cannabis use: a 4-year longitudinal and prospective study. Biological psychiatry, 71(8), 684-692.

Cloitre, M., Courtois, C. A., Charuvastra, A., Carapezza, R., Stolbach, B. C., \& Green, B. L. (2011). Treatment of complex PTSD: Results of the ISTSS expert clinician survey on best practices. Journal of Traumatic Stress, $24(6), 615-627$.

Cocozza, J. J., Jackson, E. W., Hennigan, K., Morrissey, J. P., Reed, B. G., Fallot, R., \& Banks, S. (2005). Outcomes for women with co-occurring disorders and trauma: Program-level effects. Journal of Substance Abuse Treatment, 28(2), 109-119.

Coffey, S. F., Read, J. P., \& Norberg, M. M. (2008). Posttraumatic stress disorder and substance use disorder: Neuroimaging, neuroendocrine, and psychophysiological findings. In S. Stewart \& P. Conrod (Eds.), Anxiety and Substance Use Disorders: The Vicious Cycle of Comorbidity (pp. 37-57). New York: Springer. 
Coffey, S. F., Saladin, M. E., Drobes, D. J., Brady, K. T., Dansky, B. S., \& Kilpatrick, D. G. (2002). Trauma and substance cue reactivity in individuals with comorbid posttraumatic stress disorder and cocaine or alcohol dependence. Drug and Alcohol Dependence, 65(2), 115-127.

Coffey, S. F., Saladin, M. E., Libet, J. M., Drobes, D. J., \& Dansky, B. S. (1999). Differential urge and salivary responsivity to alcohol cues in alcohol-dependent patients: A comparison of traditional and stringent classification approaches. Experimental and Clinical Psychopharmacology, 7(4), 464.

Coffey, S. F., Schumacher, J. A., Stasiewicz, P. R., Henslee, A. M., Baillie, L. E., \& Landy, N. (2010). Craving and physiological reactivity to trauma and alcohol cues in posttraumatic stress disorder and alcohol dependence. Experimental and Clinical Psychopharmacology, 18(4), 340.

Coffey, S. F., Stasiewicz, P. R., Hughes, P. M., \& Brimo, M. L. (2006). Trauma-focused imaginal exposure for individuals with comorbid posttraumatic stress disorder and alcohol dependence: Revealing mechanisms of alcohol craving in a cue reactivity paradigm. Psychology of Addictive Behaviors, 20(4), 425.

Creamer, M., Burgess, P., \& McFarlane, A. C. (2001). Post-traumatic stress disorder: findings from the Australian National Survey of Mental Health and Well-being. Psychological Medicine, 31(07), 1237-1247.

Dallman, M. F., Pecoraro, N., Akana, S. F., La Fleur, S. E., Gomez, F., Houshyar, H., ... \& Manalo, S. (2003). Chronic stress and obesity: a new view of "comfort food". Proceedings of the National Academy of Sciences, 100(20), 11696-11701. doi: 10.1073/pnas.1934666100

Dansky, B. S., Roitzsch, J. C., Brady, K. T., \& Saladin, M. E. (1997). Posttraumatic stress disorder and substance abuse: Use of research in a clinical setting. Journal of Traumatic Stress, 10(1), 141-148. doi: $10.1002 /$ jts. 2490100114

Dansky, B. S., Saladin, M. E., Coffey, S. F., \& Brady, K. T. (1997). Use of self-report measures of crime-related posttraumatic stress disorder with substance use disordered patients. Journal of Substance Abuse Treatment, 14(5), 431-437.

Darves-Bornoz, J. M., Alonso, J., de Girolamo, G., Graaf, R. D., Haro, J. M., Kovess-Masfety, V., ... \& Gasquet, I. (2008). Main traumatic events in Europe: PTSD in the European study of the epidemiology of mental disorders survey. Journal of Traumatic Stress, 21(5), 455-462.

Dass-Brailsford, P., \& Myrick, A. C. (2010). Psychological trauma and substance abuse: The need for an integrated approach. Trauma, Violence, \& Abuse, 11(4), 202-213. doi:10.1177/1524838010381252

Davis-Kean, P. E. (2005). The influence of parent education and family income on child achievement: the indirect role of parental expectations and the home environment. Journal of Family Psychology, 19(2), 294.

Dawes, R.M., Faust, D., \& Meehl, P.E. (1989). Clinical versus actuarial judgment. Science, 243(4899), 1668-1674.

de Vries, G. J., \& Olff, M. (2009). The lifetime prevalence of traumatic events and posttraumatic stress disorder in the Netherlands. Journal of Traumatic Stress, 22(4), 259-267.

Dorrepaal E, Thomaes K, Smit JH, van Balkom AJ, Veltman DJ, Hoogendoorn AW, Draijer N. (2012). Stabilizing group treatment for complex posttraumatic stress disorder related to child abuse based on psychoeducation 
and cognitive behavioural therapy: a multisite randomized controlled trial. Psychotherapy and Psychosomatics, 81(4), 217-225. doi: 10.1159/000335044.

Dragan, M., \& Lis-Turlejska, M. (2007). Prevalence of posttraumatic stress disorder in alcohol dependent patients in Poland. Addictive Behaviors, 32(5), 902-911.

Driessen, M., Schulte, S., Luedecke, C., Schaefer, I., Sutmann, F., Ohlmeier, M., ... \& Havemann-Reinicke, U. (2008). Trauma and PTSD in patients with alcohol, drug, or dual dependence: A multi-center study. Alcoholism: Clinical and Experimental Research, 32(3), 481-488.

Dube, S. R., Anda, R. F., Felitti, V. J., Chapman, D. P., Williamson, D. F., \& Giles, W. H. (2001). Childhood abuse, household dysfunction, and the risk of attempted suicide throughout the life span: findings from the Adverse Childhood Experiences Study. The Journal of the American Medical Association, 286(24), 30893096.

Ducharme, L. J., Knudsen, H. K., \& Roman, P. M. (2006). Availability of integrated care for co-occurring substance abuse and psychiatric conditions. Community Mental Health Journal, 42(4), 363-375.

Emmelkamp, P., \& Vedel, E. (2007). Alcohol- en drugsverslaving. Een gids voor effectief gebleken behandelingen. Amsterdam: Nieuwezijds.

Etkin, A., Egner, T., \& Kalisch, R. (2011). Emotional processing in anterior cingulate and medial prefrontal cortex. Trends in Cognitive Sciences, 15(2), 85-93.

Euston, D. R., Gruber, A. J., \& McNaughton, B. L. (2012). The role of medial prefrontal cortex in memory and decision making. Neuron, 76(6), 1057-1070.

Evren, C., Evren, B., Dalbudak, E., Ozcelik, B., \& Oncu, F. (2009). Childhood abuse and neglect as a risk factor for alexithymia in adult male substance dependent inpatients. Journal of Psychoactive Drugs, 41(1), 85-92.

Fareed, A., Eilender, P., Haber, M., Bremner, J., Whitfield, N., \& Drexler, K. (2013). Comorbid posttraumatic stress disorder and opiate addiction: A literature review. Journal of Addictive Diseases, 32(2), 168-179.

Farley, M., Golding, J. M., Young, G., Mulligan, M., \& Minkoff, J. R. (2004). Trauma history and relapse probability among patients seeking substance abuse treatment. Journal of Substance Abuse Treatment, 27(2), 161167.

Fattore, L., Altea, S., \& Fratta, W. (2008). Sex differences in drug addiction: a review of animal and human studies. Women's Health, 4(1), 51-65. doi: 10.2217/17455057.4.1.51

Ferry, T. Fouad, N. A., \& Smith, P. L. (2000). The role of family context in a social cognitive model for career-related choice behavior: A math and science perspective. Journal of Vocational Behavior, 57(3), 348-364.

Flint, T. A. (1992). Parental and planning influences on the formation of student college choice sets. Research in Higher Education, 33(6), 689-708.

Foa, E. B., Keane, T. M., Friedman, M. J., \& Cohen, J. A. (Eds.). (2008). Effective treatments for PTSD: practice guidelines from the International Society for Traumatic Stress Studies. London: Guilford Press. 
Forsner, T., Hansson, J., Brommels, M., Wistedt, A.A., \& Forsell, Y. (2010). Implementing clinical guidelines in psychiatry: a qualitative study of perceived facilitators and barriers. BMC Psychiatry, 10: 8.

Fox, H. C., Bergquist, K. L., Hong, K. I., \& Sinha, R. (2007). Stress-induced and alcohol cue-induced craving in recently abstinent alcohol-dependent individuals. Alcoholism: Clinical and Experimental Research, 31(3), 395-403.

Gallie, D., Paugam, S., \& Jacobs, S. (2003). Unemployment, poverty and social isolation: Is there a vicious circle of social exclusion? European Societies, 5(1), 1-32.

Garb, H.N. (2005). Clinical judgment and decision making. Annual Review of Clinical Psychology, 1, 67-89.

Garnefski, N., \& Kraaij, V. (2006). Cognitive emotion regulation questionnaire-development of a short 18-item version (CERQ-short). Personality and Individual Differences, 41(6), 1045-1053.

Gesthuizen, M., \& Scheepers, P. (2010). Economic vulnerability among low-educated Europeans: Resource, composition, labour market and welfare state influences. Acta Sociologica, 53(3), 247-267.

Gielen, N., Havermans, R. C., Tekelenburg, M., \& Jansen, A. (2012). Prevalence of post-traumatic stress disorder among patients with substance use disorder: It is higher than clinicians think it is. European Journal of Psychotraumatology, 3. doi:10.3402/ejpt.v3i0.17734

Gielen, N., Krumeich, A., Havermans, R. C., Smeets, F., \& Jansen, A. (2014). Why clinicians do not implement integrated treatment for comorbid substance use disorder and posttraumatic stress disorder: A qualitative study. European Journal of Psychotraumatology, 5. doi:10.3402/ejpt.v5.22821

Gielen, N., Krumeich, A., Tekelenburg, M., Nederkoorn, C., \& Havermans, R.C. (2015a). Coping skills, motives for substance use and symptom interplay: how patients with substance use disorder and PTSD experience their reality. Manuscript submitted for publication.

Gielen, N., Nederkoorn, C. \& Havermans, R. C. (2015b). How patients perceive the relationship between trauma, substance abuse, craving, and relapse: A qualitative study. Journal of Substance Use. http://dx.doi.org/10.3109/14659891.2015.1063717

Gielen, N., Nederkoorn, C., Jansen, A., \& Havermans, R. C. Cued cravings in substance dependent patients with posttraumatic stress disorder. Manuscript submitted for publication.

Glover-Graf, N. M., \& Janikowski, T. P. (2001). Substance abuse counsellors' experiences with victims of incest. Journal of Substance Abuse Treatment, 20, 9-14.

Grant, V. V., Stewart, S. H., O'Connor, R. M., Blackwell, E., \& Conrod, P. J. (2007). Psychometric evaluation of the five-factor Modified Drinking Motives Questionnaire-Revised in undergraduates. Addictive Behaviors, 32(11), 2611-2632.

Gross, A. M., Winslett, A., Roberts, M., \& Gohm, C. L. (2006). An examination of sexual violence against college women. Violence Against Women, 12(3), 288-300.

Hall, J. M. (2000). Core issues for female child abuse survivors in recovery from substance misuse. Qualitative Health Research, 10(5), 612-631. doi:10.1177/104973230001000504 
Haller, M., \& Chassin, L. (2014). Risk pathways among traumatic stress, posttraumatic stress disorder symptoms, and alcohol and drug problems: A test of four hypotheses. Psychology of Addictive Behaviors, 28(3), 841851.

Harmon-Jones, E., Sigelman, J., Bohlig, A., \& Harmon-Jones, C. (2003). Anger, coping, and frontal cortical activity: The effect of coping potential on anger-induced left frontal activity. Cognition \& Emotion, 17(1), 1-24.

Harris, M., Fallot, R. D., \& Berley, R. W. (2005). Special section on relapse prevention: Qualitative interviews on substance abuse relapse and prevention among female trauma survivors. Psychiatric Services, 56(10), 1292-1296. doi:10.1176/appi.ps.56.10.1292

Henslee, A.M., \& Coffey, S.F. (2010). Exposure therapy for posttraumatic stress disorder in a residential substance use treatment facility. Professional Psychology, Research and Practice, 1, 34-40.

Hien, D. A., Wells, E. A., Jiang, H., Suarez-Morales, L., Campbell, A. N., Cohen, L. R., ... \& Nunes, E. V. (2009). Multisite randomized trial of behavioral interventions for women with co-occurring PTSD and substance use disorders. Journal of Consulting and Clinical Psychology, 77(4), 607-619.

Hien, D. A., Cohen, L. R., \& Campbell, A. (2005). Is traumatic stress a vulnerability factor for women with substance use disorders?. Clinical Psychology Review, 25(6), 813-823.

Hien, D. A., Cohen, L. R., Miele, G. M., Litt, L. C., \& Capstick, C. (2004). Promising treatments for women with comorbid PTSD and substance use disorders. The American Journal of Psychiatry, 161, 1426-1432.

Hildebrand, A., Behrendt, S., \& Hoyer, J. (2015). Treatment outcome in substance use disorder patients with and without comorbid posttraumatic stress disorder: A systematic review. Psychotherapy Research, 25(5), $565-582$

Hovens, J. E., Bramsen, I., \& Van der Ploeg, H. M. (2002). Self-rating inventory for posttraumatic stress disorder: review of the psychometric properties of a new brief Dutch screening instrument. Perceptual and Motor Skills, 94(3), 996-1008.

Hovens, J. E., Van der Ploeg, H., Bramsen, I., Klaarenbeek, M. T. A., Schreuder, J. N., \& Rivero, V. V. (1994). The development of the self-rating inventory for posttraumatic stress disorder. Acta Psychiatrica Scandinavica, 90(3), 172-183.

Jacobsen, L. K., Southwick, S. M., \& Kosten, T. R. (2001). Substance use disorders in patients with posttraumatic stress disorder: a review of the literature. American Journal of Psychiatry, 158, 1184-1190.

Janikowski, T. P., \& Glover, N. M. (1994). Incest and substance abuse: Implications for treatment professionals. Journal of Substance Abuse Treatment, 11(3), 177-183. doi:10.1016/0740-5472(94)90074-4

Janikowski, T. P., Bordieri, J. E., \& Glover, N. M. (1997). Client perceptions of incest and substance abuse. Addictive Behaviors, 22(4), 447-459. doi:10.1016/s0306-4603(97)84428-0

Janikowski, T.P., \& Glover-Graf, N.M. (2003). Qualifications, training, and perceptions of substance abuse counsellors who work with victims of incest. Addictive Behaviors, 28, 1193-1201. 
Jarvis, T. J., Copeland, J., \& Walton, L. (1998). Exploring the nature of the relationship between child sexual abuse and substance use among women. Addiction, 93(6), 865-875. doi:10.1046/j.1360-0443.1998.9368658.x

Jaycox, L. H., Foa, E. B., \& Morral, A. R. (1998). Influence of emotional engagement and habituation on exposure therapy for PTSD. Journal of Consulting and Clinical Psychology, 66(1), 185-192.

Johnson, S. D. (2008). Substance use, post-traumatic stress disorder and violence. Current Opinion in Psychiatry, 21(3), 242-246.

Keane, T. M., Marshall, A. D., \& Taft, C. T. (2006). Posttraumatic stress disorder: etiology, epidemiology, and treatment outcome. Annual Review of Clinical Psychology, 2, 161-197.

Kessler, R. C., Chiu, W. T., Demler, O., \& Walters, E. E. (2005). Prevalence, severity, and comorbidity of 12-month DSM-IV disorders in the National Comorbidity Survey Replication. Archives of General Psychiatry, 62(6), 617-627.

Khantzian, E. J. (1997). The self-medication hypothesis of substance use disorders: a reconsideration and recent applications. Harvard Review of Psychiatry, 4(5), 231-244.

Killeen, T. K., Back, S. E., \& Brady, K. T. (2015). Implementation of integrated therapies for comorbid post-traumatic stress disorder and substance use disorders in community substance abuse treatment programs. Drug and Alcohol Review, 34(3), 234-241.

Kivlahan, D., \& Kaysen, D. (2012). Managing PTSD and co-occurring substance use disorders. PTSD 101 Course Module. National Centers for PTSD Online Training Program. Retrieved from http://www.ptsd.va.gov/professional/ptsd101/course-modules/SUD.asp

Klaassens, E. R., Giltay, E. J., Cuijpers, P., van Veen, T., \& Zitman, F. G. (2012). Adulthood trauma and HPA-axis functioning in healthy subjects and PTSD patients: a meta-analysis. Psychoneuroendocrinology, 37(3), 317331.

Klaassens, E. R., van Noorden, M. S., Giltay, E. J., van Pelt, J., van Veen, T., \& Zitman, F. G. (2009). Effects of childhood trauma on HPA-axis reactivity in women free of lifetime psychopathology. Progress in NeuroPsychopharmacology and Biological Psychiatry, 33(5), 889-894.

Klanecky, A. K., Harrington, J., \& McChargue, D. E. (2008). Child sexual abuse, dissociation, and alcohol: Implications of chemical dissociation via blackouts among college women. The American Journal of Drug and Alcohol Abuse, 34(3), 277-284.

Kleinman, A. (1980). Patients and healers in the context of culture: an exploration of the borderland between anthropology, medicine and psychiatry. Berkeley: University of California Press.

Kok, T., de Haan, H. A., van der Velden, H. J., van der Meer, M., Najavits, L. M., \& de Jong, C. A. (2013). Validation of two screening instruments for PTSD in Dutch substance use disorder inpatients. Addictive behaviors, 38(3), 1726-1731. 
Kwako, L. E., Schwandt, M. L., Sells, J. R., Ramchandani, V. A., Hommer, D. W., George, D. T., ... \& Heilig, M. (2014). Methods for inducing alcohol craving in individuals with co-morbid alcohol dependence and posttraumatic stress disorder: behavioral and physiological outcomes. Addiction Biology, 20(4), 733-746.

LaCoursiere, R. B., Godfrey, K. E., \& Ruby, L. M. (1980). Traumatic neurosis in the etiology of alcoholism: Viet Nam combat and other trauma. The American Journal of Psychiatry, 137(8), 966-968. doi:10.1176/ajp.137.8.966

Langeland, W. (2009). Trauma. In I. Franken, I., \& W. van den Brink (Eds.), Handboek verslaving [Guide to addiction] (pp.135-150). Utrecht: De Tijdstroom.

Langeland, W., Draijer, N., \& van den Brink, W. (2002). Trauma and dissociation in treatment-seeking alcoholics: towards a resolution of inconsistent findings. Comprehensive Psychiatry, 43(3), 195-203.

Larrabee, J. H., \& Bolden, L. V. (2001). Defining patient-perceived quality of nursing care. Journal of Nursing Care Quality, 16(1), 34-60. doi:10.1097/00001786-200110000-00005

Leeies, M., Pagura, J., Sareen, J., \& Bolton, J.M. (2010). The use of alcohol and drugs to self-medicate symptoms of posttraumatic stress disorder. Depression and Anxiety, 27, 731-736.

Leentjens, A.F., \& Burgers, J.S. (2008). Welke factoren zijn belangrijk bij succesvolle implementatie van richtlijnen? [What factors are important for the successful implementation of guidelines?]. Tijdschrift voor Psychiatrie, 50, 329-335.

Leskin, L. P., \& White, P. M. (2007). Attentional networks reveal executive function deficits in posttraumatic stress disorder. Neuropsychology, 21(3), 275-284.

Liem, R., \& Rayman, P. (1982). Health and social costs of unemployment: Research and policy considerations. American Psychologist, 37(10), 1116-1123.

Lobbestael, J., Arntz, A., Harkema-Schouten, P., \& Bernstein, D. (2009). Development and psychometric evaluation of a new assessment method for childhood maltreatment experiences: The interview for traumatic events in childhood (ITEC). Child Abuse \& Neglect, 33(8), 505-517.

Lupien, S. J., McEwen, B. S., Gunnar, M. R., \& Heim, C. (2009). Effects of stress throughout the lifespan on the brain, behaviour and cognition. Nature Reviews Neuroscience, 10(6), 434-445.

Maier, S. F., \& Watkins, L. R. (2010). Role of the medial prefrontal cortex in coping and resilience. Brain research, 1355, 52-60.

McCauley, J. L., Killeen, T., Gros, D. F., Brady, K. T., \& Back, S. E. (2012). Posttraumatic stress disorder and cooccurring substance use disorders: Advances in assessment and treatment. Clinical Psychology: Science and Practice, 19(3), 283-304.

McConnell, M. M., Memetovic, J., \& Richardson, C. G. (2014). Coping style and substance use intention and behavior patterns in a cohort of BC adolescents. Addictive Behaviors, 39(10), 1394-1397. 
McEwen, B. S., \& Seeman, T. (1999). Protective and damaging effects of mediators of stress: elaborating and testing the concepts of allostasis and allostatic load. Annals of the New York Academy of Sciences, 896(1), 30-47.

McFall, M., Saxon, A. J., Malte, C. A., Chow, B., Bailey, S., Baker, D. G., ... \& CSP 519 Study Team. (2010). Integrating tobacco cessation into mental health care for posttraumatic stress disorder: a randomized controlled trial. The Journal of the American Medical Association, 304(22), 2485-2493.

McHugo, G.J., Caspi, Y., Kammerer, N., Mazelis, R., Jackson, E.W., Russell, L., . . . Kimerling, R. (2005). The assessment of trauma history in women with co-occurring substance abuse and mental disorders and a history of interpersonal violence. Journal of Behavioral Health Services \& Research, 32(2), 113-127.

Mills, K. L., Lynskey, M., Teesson, M., Ross, J., \& Darke, S. (2005). Post-traumatic stress disorder among people with heroin dependence in the Australian treatment outcome study (ATOS): prevalence and correlates. Drug and Alcohol Dependence, 77(3), 243-249.

Mills, K. L., Teesson, M., Ross, J., \& Peters, L. (2006). Trauma, PTSD, and substance use disorders: findings from the Australian National Survey of Mental Health and Well-Being. The American Journal of Psychiatry, 163(4), 652-658.

Mills, K. L., Teesson, M., Back, S. E., Brady, K. T., Baker, A. L., Hopwood, S., . . . Ewer, P. L. (2012). Integrated exposure-based therapy for co-occurring posttraumatic stress disorder and substance dependence: a randomized controlled trial. The Journal of the American Medical Association, 15, 690-699.

Mills, K. L., Teesson, M., Ross, J., Darke, S., \& Shanahan, M. (2005). The costs and outcomes of treatment for opioid dependence associated with posttraumatic stress disorder. Psychiatric Services, 56, 940-945.

Morrissey, J. P., Ellis, A. R., Gatz, M., Amaro, H., Reed, B. G., Savage, A., ... \& Banks, S. (2005). Outcomes for women with co-occurring disorders and trauma: Program and person-level effects. Journal of Substance Abuse Treatment, 28(2), 121-133.

Mueser, K. T., Noordsy, D. L., Drake, R. E., \& Fox, L. (2003). Integrated treatment for dual disorders: a guide to effective practice. New York: Guilford Press.

Najavits, L. M. (2005). Theoretical perspective on posttraumatic stress disorder and substance use disorder. Australian Psychologist, 40(2), 118-126.

Najavits, L. M., \& Hien, D. (2013). Helping vulnerable populations: a comprehensive review of the treatment outcome literature on substance use disorder and PTSD. Journal of Clinical Psychology, 69(5), 433-479.

Najavits, L. M., Weiss, R. D., Shaw, S. R., \& Muenz, L. R. (1998). "Seeking safety": Outcome of a new cognitivebehavioral psychotherapy for women with posttraumatic stress disorder and substance dependence. Journal of Traumatic Stress, 11(3), 437-456.

Najavits, L. M. (2002). Clinicians' views on treating posttraumatic stress disorder and substance use disorder. Journal of Substance Abuse Treatment, 22, 79-85. 
Najavits, L. M., Gallop, R. J., \& Weiss, R. D. (2006). Seeking safety therapy for adolescent girls with PTSD and substance use disorder: a randomized controlled trial. The Journal of Behavioral Health Services and Research, 33, 453-463.

Najavits, L. M., Norman, S. B., Kivlahan, D., \& Kosten, T. R. (2010). Improving PTSD/ substance abuse treatment in the VA: a survey of providers. The American Journal on Addictions, 19, 257-263.

Najavits, L. M., Sullivan, T. P., Schmitz, M., Weiss, R. D., \& Lee, C. S. (2004). Treatment utilization by women with PTSD and substance dependence. The American Journal on Addictions, 13, 215-224.

Najt, P., Fusar-Poli, P., \& Brambilla, P. (2011). Co-occurring mental and substance abuse disorders: a review on the potential predictors and clinical outcomes. Psychiatry Research, 30, 159-164.

Nijenhuis, E. R., Van der Hart, O., \& Kruger, K. (2002). The psychometric characteristics of the Traumatic Experiences Checklist (TEC): First findings among psychiatric outpatients. Clinical Psychology \& Psychotherapy, 9(3), 200-210.

Norman, S. B., Inaba, R. K., Smith, T. L., \& Brown, S. A. (2008). Development of the PTSD-alcohol expectancy questionnaire. Addictive Behaviors, 33(6), 841-847.

Norman, S. B., Tate, S. R., Anderson, K. G., \& Brown, S. A. (2007). Do trauma history and PTSD symptoms influence addiction relapse context? Drug and Alcohol Dependence, 90(1), 89-96.

Nosen, E., Littlefield, A. K., Schumacher, J. A., Stasiewicz, P. R., \& Coffey, S. F. (2014). Treatment of co-occurring PTSD-AUD: Effects of exposure-based and non-trauma focused psychotherapy on alcohol and trauma cue-reactivity. Behaviour Research and Therapy, 61, 35-42.

Nosen, E., Nillni, Y. I., Berenz, E. C., Schumacher, J. A., Stasiewicz, P. R., \& Coffey, S. F. (2012). Cue-elicited affect and craving: Advancement of the conceptualization of craving in co-occurring posttraumatic stress disorder and alcohol dependence. Behavior Modification, 36, 808-833.

Ooteman, W., Koeter, M., Verheul, R., Schippers, G., \& Van Den Brink, W. (2006). Development and validation of the Amsterdam Motives for Drinking Scale (AMDS): an attempt to distinguish relief and reward drinkers. Alcohol and Alcoholism, 41(3), 284-292.

Ouimette, P. C., Brown, P. J., \& Najavits, L. M. (1998). Course and treatment of patients with both substance use and posttraumatic stress disorders. Addictive Behaviors, 23(6), 785-795. doi:10.1016/s03064603(98)00064-1

Ouimette, P. C., Coolhart, D., Funderburk, J. S., Wade, M., \& Brown, P. J. (2007). Precipitants of first substance use in recently abstinent substance use disorder patients with PTSD. Addictive Behaviors, 32(8), 1719-1727. doi:10.1016/j.addbeh.2006.11.020

Ouimette, P. C., Finney, J. W., \& Moos, R. H. (1999). Two-year posttreatment functioning and coping of substance abuse patients with posttraumatic stress disorder. Psychology of Addictive Behaviors, 13(2), 105-114. doi:10.1037//0893-164x.13.2.105 
Ouimette, P. C., Goodwin, E., \& Brown, P. J. (2006). Health and well being of substance use disorder patients with and without posttraumatic stress disorder. Addictive Behaviors, 31(8), 1415-1423.

Ouimette, P. C., Read, J. P., Wade, M., \& Tirone, V. (2010). Modelling associations between posttraumatic stress symptoms and substance use. Addictive Behaviors, 35, 64-67.

Overbeek, I., Schruers, K., \& Griez, E. (1999). Mini international neuropsychiatric interview: Nederlandse versie 5.0. 0. DSM-IV [Dutch version]. Maastricht, The Netherlands: Universiteit Maastricht.

Pagura, J., Stein, M. B., Bolton, J. M., Cox, B. J., Grant, B., \& Sareen, J. (2010). Comorbidity of borderline personality disorder and posttraumatic stress disorder in the US population. Journal of Psychiatric Research, 44(16), 1190-1198.

Palmer, M., Hayek, J., Hossler, D., Jacob, S. A., Cummings, H., \& Kinzie, J. (2004). Fifty years of college choice: Social, political and institutional influences on the decision-making process. Lumina: New Agenda Series, 5(3), 1-65.

Paul, K. I., \& Moser, K. (2009). Unemployment impairs mental health: Meta-analyses. Journal of Vocational Behavior, 74(3), 264-282.

Peirce, J. M., Kindbom, K. A., Waesche, M. C., Yuscavage, A. S., \& Brooner, R. K. (2008). Posttraumatic stress disorder, gender, and problem profiles in substance dependent patients. Substance Use \& Misuse, 43(5), 596-611.

Pirard, S., Sharon, E., Kang, S. K., Angarita, G. A., \& Gastfriend, D. R. (2005). Prevalence of physical and sexual abuse among substance abuse patients and impact on treatment outcomes. Drug and alcohol dependence, 78(1), 57-64.

Pope, C., Ziebland, S., \& Mays, N. (2000). Qualitative research in health care: Analysing qualitative data. BMJ: British Medical Journal, 320(7227), 114-116. doi:10.1136/bmj.320.7227.114

Read, J. P., Brown, P. J., \& Kahler, C. W. (2004). Substance use and posttraumatic stress disorders: symptom interplay and effects on outcome. Addictive Behaviors, 29, 1665-1672.

Reed, P. L., Anthony, J. C., \& Breslau, N. (2007). Incidence of drug problems in young adults exposed to trauma and posttraumatic stress disorder: do early life experiences and predispositions matter? Archives of General Psychiatry, 64(12), 1435-1442.

Reynolds, M., Mezey, G., Chapman, M., Wheeler, M., \& Baldacchino, A. (2005). Co-morbid post-traumatic stress disorder in a substance misusing clinical population. Drug and Alcohol Dependence, 7, 251-258.

Roberts, M. E., Moore, S., D., \& Beckham, J. C. (2007). Post-traumatic stress disorder and substance use disorders. In M. Al'Absi (Ed.), Stress and addiction. Biological and psychological mechanisms (pp. 315-331). London, UK: Elsevier.

Rothkrauf, T. C., \& Eby, L. T. (2011). Counsellors' knowledge of the adoption of tobacco cessation medications in substance abuse treatment programs. The American Journal on Addictions, 20, 56-62. 
Ruzek, J. I., Polusny, M. A., \& Abueg, F. R. (1998). Assessment and treatment of concurrent posttraumatic stress disorder and substance abuse. In V. M. Follette, J. I. Ruzek, \& F. R. Abueg (Eds), Cognitive-Behavioral Therapies for Trauma (pp. 226-255). New York: Guilford Press.

Saladin, M. E., Drobes, D. J., Coffey, S. F., Dansky, B. S., Brady, K. T., \& Kilpatrick, D. G. (2003). PTSD symptom severity as a predictor of cue-elicited drug craving in victims of violent crime. Addictive Behaviors, 28(9), 1611-1629.

Schatzberg, A. F., Weiss, R. D., Brady, K. T., \& Culpepper, L. (2008). Bridging the clinical gap: managing patients with co-occurring mood, anxiety, and alcohol use disorders. Introduction. CNS Spectrums, 13: 3.

Schneekloth, T. D., Biernacka, J. M., Hall-Flavin, D. K., Karpyak, V. M., Frye, M. A., Loukianova, L. L., ... \& Mrazek, D. A. (2012). Alcohol craving as a predictor of relapse. The American Journal on Addictions, 21(Suppl. 1), 2026.

Sewell, W. H., \& Shah, V. P. (1968). Social class, parental encouragement, and educational aspirations. American Journal of Sociology, 559-572.

Shalev, A. Y., Freedman, S., Peri, T., Brandes, D., Sahar, T., Orr, S. P., \& Pitman, R. K. (1998). Prospective study of posttraumatic stress disorder and depression following trauma. American Journal of Psychiatry, 155, 630637.

Sheehan, D. V., Lecrubier, Y., Sheehan, K. H., Janavs, J., Weiller, E., Keskiner, A., ... \& Dunbar, G. C. (1997). The validity of the Mini International Neuropsychiatric Interview (MINI) according to the SCID-P and its reliability. European Psychiatry, 12(5), 232-241.

Shin, L. M., Rauch, S. L., \& Pitman, R. K. (2006). Amygdala, medial prefrontal cortex, and hippocampal function in PTSD. Annals of the New York Academy of Sciences, 1071(1), 67-79.

Simons, J., Correia, C. J., Carey, K. B., \& Borsari, B. E. (1998). Validating a five-factor marijuana motives measure: Relations with use, problems, and alcohol motives. Journal of Counseling Psychology, 45(3), 265-273.

Simpson, T. L., \& Miller, W. R. (2002). Concomitance between childhood sexual and physical abuse and substance use problems: A review. Clinical Psychology Review, 22(1), 27-77.

Sinha, R. (1996). Multivariate response patterning of fear and anger. Cognition \& Emotion, 10(2), 173-198.

Sinha, R. (2007). The role of stress in addiction relapse. Current Psychiatry Reports, 9(5), 388-395.

Sinha, R., \& Li, C. S. R. (2007). Imaging stress-and cue-induced drug and alcohol craving: association with relapse and clinical implications. Drug and Alcohol Review, 26(1), 25-31.

Smeets, F. (2009). Trauma, addiction \& treatment: a qualitative study on clinicians' perspective (unpublished master's thesis). Maastricht University, Maastricht.

Snoek, A., Wits, E., Meulders, W., \& Van de Mheen, D. (2012). Richtlijn middelenmisbruik of -afhankelijkheid en angststoornissen [Guideline anxiety disorders and substance use disorders]. Tijdschrift Verslaving [Addiction Journal], 8, 59-62. 
Sofaer, S., \& Firminger, K. (2005). Patient perceptions of the quality of health services. Annual Review of Public Health, 26(1), 513-559. doi:10.1146/annurev.publhealth.25.050503.153958

Somer, E., Altus, L., \& Ginzburg, K. (2010). Dissociative psychopathology among opioid use disorder patients: exploring the "chemical dissociation" hypothesis. Comprehensive Psychiatry, 51(4), 419-425.

Sorensen, J. L., \& Kosten, T. (2011). Developing the tools of implementation science in substance use disorders treatment: applications of the consolidated framework for implementing research. Psychology of Addictive Behaviors, 25, 262-268.

Spinhoven, P., Penninx, B. W., van Hemert, A. M., de Rooij, M., \& Elzinga, B. M. (2014). Comorbidity of PTSD in anxiety and depressive disorders: Prevalence and shared risk factors. Child Abuse \& Neglect, 38(8), 13201330.

Staiger, P. K., Melville, F., Hides, L., Kambouropoulos, N., \& Lubman, D. I. (2009). Can emotion-focused coping help explain the link between posttraumatic stress disorder severity and triggers for substance use in young adults? Journal of Substance Abuse Treatment, 36(2), 220-226. doi:10.1016/j.jsat.2008.05.008

Stewart, S. H., Pihl, R. O., Conrod, P. J., \& Dongier, M. (1998). Functional associations among trauma, PTSD, and substance-related disorders. Addictive Behaviors, 23(6), 797-812.

Stewart, S. H., \& Conrod, P. J. (2008). Anxiety and substance use disorders: The vicious cycle of comorbidity. New York: Springer.

Taber, J. I., McCormick, R. A., \& Ramirez, L. F. (1987). The prevalence and impact of major life stressors among pathological gamblers. Substance Use \& Misuse, 22(1), 71-79. doi:10.3109/10826088709027414

Teicher, M. H., Andersen, S. L., Polcari, A., Anderson, C. M., Navalta, C. P., \& Kim, D. M. (2003). The neurobiological consequences of early stress and childhood maltreatment. Neuroscience \& Biobehavioral Reviews, 27(1), 33-44.

Teusch, R. (2001). Substance abuse as a symptom of childhood sexual abuse. Psychiatric Services, 52(11), 15301532. doi:10.1176/appi.ps.52.11.1530

Torchalla, I., Nosen, L., Rostam, H., \& Allen, P. (2012). Integrated treatment programs for individuals with concurrent substance use disorders and trauma experiences: A systematic review and meta-analysis. Journal of Substance Abuse Treatment, 42(1), 65-77. doi:10.1016/j.jsat.2011.09.001

Ullman, S. E., Relyea, M., Peter-Hagene, L., \& Vasquez, A. L. (2013). Trauma histories, substance use coping, PTSD, and problem substance use among sexual assault victims. Addictive Behaviors, 38(6), 2219-2223.

Van Balkom, A. J. L. M., De Beurs, E., Hovens, J. E. J. M., \& Van Vliet, I. M. (2004). Meetinstrumenten bij angststoornissen. Tijdschrift voor Psychiatrie, 46(10), 687-692.

Van Dam, D., Ehring, T., Vedel, E., \& Emmelkamp, P. M. (2010). Validation of the Primary Care Posttraumatic Stress Disorder screening questionnaire (PC-PTSD) in civilian substance use disorder patients. Journal of Substance Abuse Treatment, 39(2), 105-113. 
Van Dam, D., Vedel, E., Ehring, T., \& Emmelkamp, P. M. (2012). Psychological treatments for concurrent posttraumatic stress disorder and substance use disorder: A systematic review. Clinical Psychology Review, 32(3), 202-214. doi:10.1016/j.cpr.2012.01.004

Van Den Bosch, L. M., Verheul, R., Langeland, W., \& Van Den Brink, W. (2003). Trauma, dissociation, and posttraumatic stress disorder in female borderline patients with and without substance abuse problems. Australian and New Zealand Journal of Psychiatry, 37(5), 549-555.

Van der Bossche, C. M. R., Kremers, I., Sieswerda, S. H., \& Arntz, A. (1999). Vragenlijst naar Belastende Gebeurtenissen (VBG)[Structured Childhood Trauma Interview]. Maastricht: Maastricht University.

Vanyukov, M. M., Tarter, R. E., Kirillova, G. P., Kirisci, L., Reynolds, M. D., Kreek, M. J., ... \& Ridenour, T. A. (2012). Common liability to addiction and "gateway hypothesis": theoretical, empirical and evolutionary perspective. Drug and Alcohol Dependence, 123, S3-S17. doi:10.1016/j.drugalcdep.2011.12.018

Vermetten, E. \& Lanius, R. A. (2012). Biological and clinical framework for posttraumatic stress disorder. In: T.E. Schlaepfer \& C.B. Nemeroff (Eds.), Handbook of clinical neurology (pp. 291 - 342). London: Elsevier.

Weathers, F. W., Keane, T. M., \& Davidson, J. R. (2001). Clinician-administered PTSD scale: a review of the first ten years of research. Depression and Anxiety, 13(3), 132-156.

Weathers, F. W., Blake, D. D., Schnurr, P. P., Kaloupek, D. G., Marx, B. P., \& Keane, T. M. (2013). The ClinicianAdministered PTSD Scale for DSM-5 (CAPS-5). Interview available from the National Center for PTSD at http://www.ptsd.va.gov.

Witteveen, A. B., Bramsen, I., Hovens, J. E., \& Van der Ploeg, H. M. (2005). Utility of the impact of event scale in screening for posttraumatic stress disorder. Psychological Reports, 97(1), 297-308.

Young, H. E., Rosen, C. S., \& Finney, J. W. (2005). A survey of PTSD screening and referral practices in VA addiction treatment programs. Journal of Substance Abuse Treatment, 28(4), 313-319. doi:10.1016/j.jsat.2005.02.006

Zatzick, D., Roy-Byrne, P., Russo, J., Rivara, F., Droesch, R., Wagner, A., ... Katon, W. (2004). A randomized effectiveness trial of stepped collaborative care for acutely injured trauma survivors. Archives of General Psychiatry, 61, 498-506. 